(C) 2007

Jin Namkoong

ALL RIGHTS RESERVED 


\title{
METABOTROPIC GLUTAMATE RECEPTOR 1 AND GLUTAMATE \\ SIGNALING IN HUMAN MELANOMA
}

\author{
by \\ JIN NAMKOONG \\ A Dissertation submitted to the \\ Graduate School-New Brunswick \\ Rutgers, The State University of New Jersey and \\ The Graduate School of Biomedical Sciences \\ University of Medicine and Dentistry of New Jersey \\ in partial fulfillment of the requirements \\ for the degree of Doctor of Philosophy \\ Graduate Program in \\ Microbiology and Molecular Genetics \\ written under the direction of \\ Dr. Suzie Chen \\ and approved by
}

New Brunswick, New Jersey

May, 2007 


\begin{abstract}
OF THE DISSERTATION
Metabotropic Glutamate Receptor 1 and Glutamate Signaling in Human Melanoma by JIN NAMKOONG

Dissertation Director: Dr. Suzie Chen
\end{abstract}

Melanoma is the most malignant form of skin cancers that originated from melanocytes, the pigment cells in the skin. Early detection is the key for increased chance of survival, since currently there is no assuring therapeutic means for late stage melanoma. Animal models have been a valuable tool to understand human disorders and to identify new therapeutic targets for the treatments of a disease including melanoma. Previously, we reported on a transgenic mouse model with predisposition to melanoma development with $100 \%$ penetrance [37]. Subsequent studies identified the aberrant expression of metabotropic glutamate receptor 1 (Grm1) in melanocytes to be critical in the onset of melanoma. Confirmation of the etiological role of Grm1 in melanoma development was demonstrated in a second transgenic mouse line with Grm1 expression under the regulation of a melanocyte-specific dopachrome tautomerase (DCT) promoter [40].

Ectopic expression of GRM1 was also detected in a subset of human melanoma cell lines and biopsies, suggesting that aberrant expression of GRM1 in melanocytes may contribute to the development of human melanoma. GRM1, a seven-transmembrane domain G-protein coupled receptor, is normally expressed and functional in neuronal cells and its ligand, glutamate, is the major excitatory neurotransmitter. MAPK has been 
shown by many investigators to be one of the key signaling pathways in human melanoma cell proliferation [45, 55]; We also showed that MAPK signaling cascade being the downstream target of activated GRM1 [47]. Furthermore, treatment of GRM1expressing human melanoma cells with a GRM1-antagonist leads to a suppression of cell proliferation. Human melanoma cells released elevated levels of glutamate, implying a possible autocrine loop. Treatment of human melanoma cells with a GRM1-antagonist or an inhibitor of glutamate release, Riluzole, leads to a decrease in release of glutamate and suppression of cell growth. In vivo xenografts with human melanoma cells, when treated with Riluzole, resulted in an inhibition of tumor growth/progression. Taken together, these data suggest the pivotal role of glutamate signaling in human melanoma and imply the potential of a new target for melanoma therapy. 


\section{ACKNOWLEDGEMENT}

I would like to express my gratitude to my advisor, Dr. Suzie Chen, for being a great mentor. She taught me more than just science. I am a better scientist and person because of her teaching and patience.

I would like to thank Dr. David Axelrod, Dr. James Goydos, and Dr. Renping Zhou for helpful discussions and being my committee members.

I would like to also thank members of Chen Laboratory, colleagues and friends at Susan Lehman Cullman Laboratory for Cancer Research and the K.M. members. They helped me with my research and made my day enjoyable. 


\section{DEDICATION}

This dissertation is dedicated to my family and my friends, whose love and support carried me this far. 


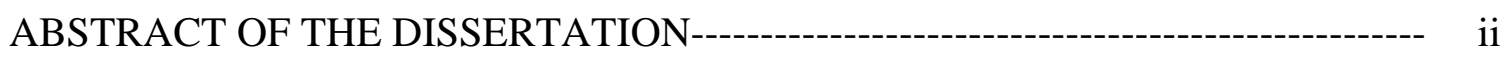

ACKNOWLEDGEMENT---

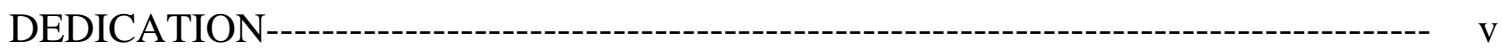

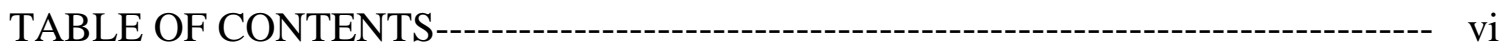

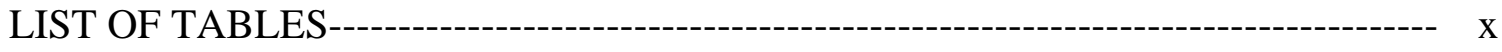

LIST OF FIGURES--

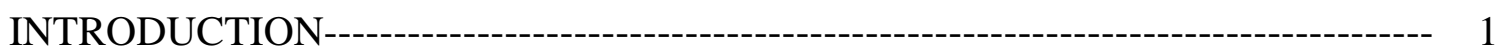

PART I: SKIN CANCER---_-_-_-_-_-- 1

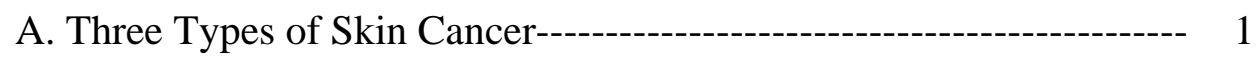

B. Melanoma------------------------------------------------------------------- 3

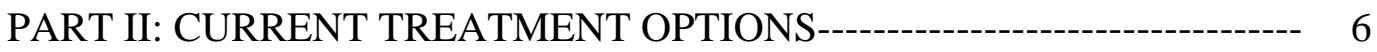

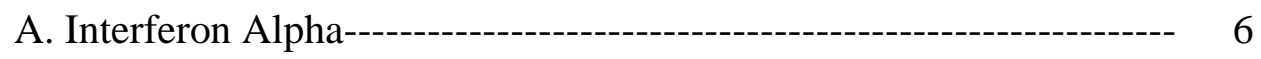

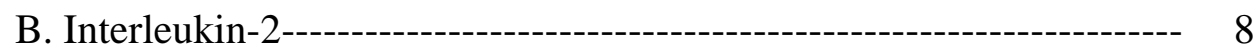

C. Others----_- 9

PART III: METABOTROPIC GLUTAMATE RECEPTOR 1 (GRM1)-------- 12

A. Mouse Model for Melanoma---------------------------------------------- 12

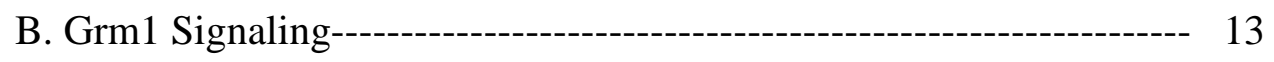

PART IV: MELANOMA CELL PROLIFERATION PATHWAY AND ITS

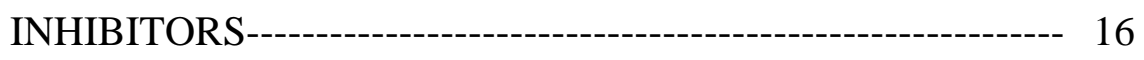

A. RAS--------_--- 16 


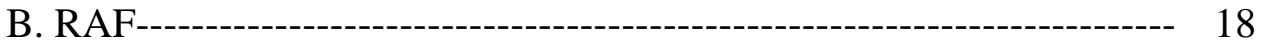

C. MEK/ERK------ 22

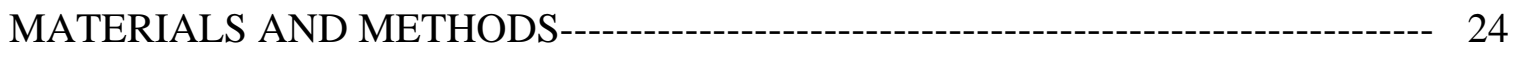

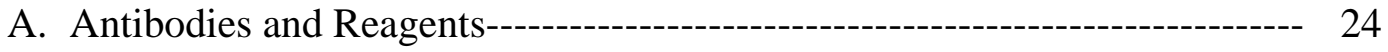

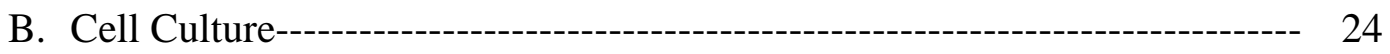

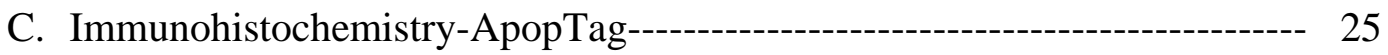

D. Immunocytochemistry--------------------------------------------------------- 26

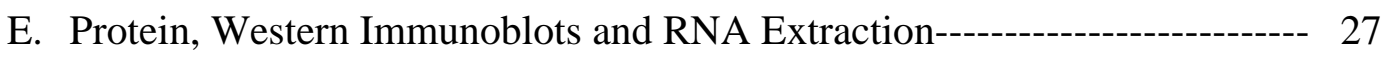

F. Inositol-1,4,5-triphosphate Measurements--------------------------------------- 28

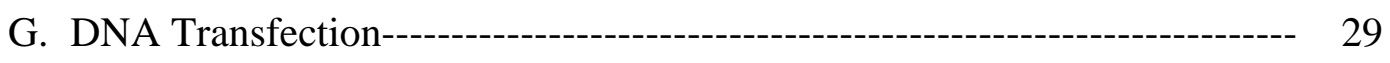

H. MTT Cell Proliferation Assays----------------------------------------------------- 30

I. Measurement of Extracellular Glutamate----------------------------------------- 30

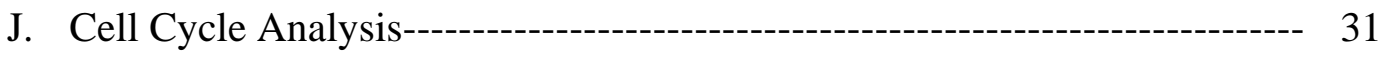

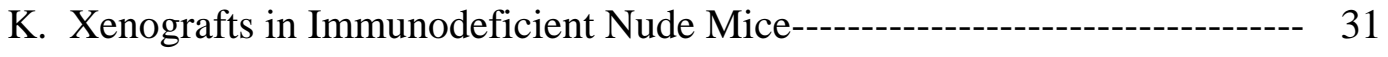

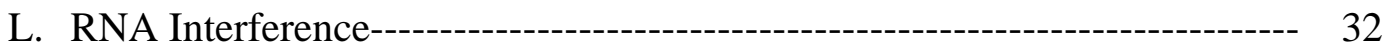

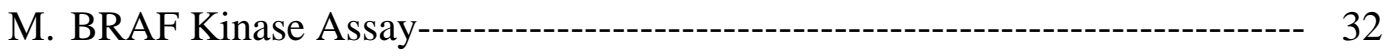

RESULTS---

PART I: HUMAN MELANOMA AND GRM1----------------------------------- 34

A. GRM1 Expression in Human Melanoma Biopsy Samples------------ 34

B. GRM1 Expression in Human Melanoma Cell Lines------------------- 36

C. Functional GRM1 in Human Melanoma Cells-------------------------- 37

PART II: SUPPRESSION OF GRM1 SIGNALING------------------------------- 41

A. Induction of Apoptosis by Dominant Negative Mutants of GRM1-- 41 
B. Inhibition of Human Melanoma Cell Proliferation by GRM1

Antagonists

1. LY367385, the Competitive Antagonist of GRM1----------- 42

2. BAY 36-7520, the Non-Competitive Antagonist of GRM1-- 43

C. Suppression of GRM1 Expression by RNA Interference (RNAi)---- 45

PART III: GLUTAMATE SIGNALING IN MELANOMA--------------------- 47

A. Glutamate Release in Melanoma------------------------------------- 47

B. Inhibition of Glutamate Release by GRM1 Antagonists in Human

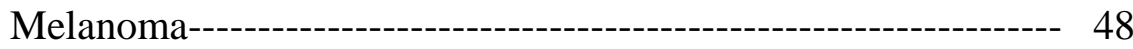

C. Inhibition of Cell Proliferation by the Glutamate Release Inhibitor,

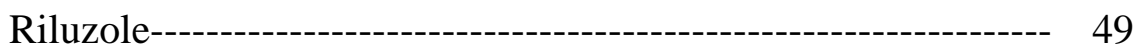

D. Inhibition of Human Melanoma Xenograft Growth by Riluzole----- 50

PART IV: UACC930, A GRM1-NEGATIVE HUMAN MELANOMA CELL

LINE-

PART V: INVOLVEMENT OF BRAF KINASE IN GRM1 SIGNALING IN MOUSE MELANOMA

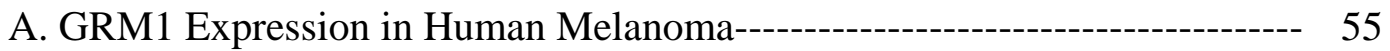

B. GRM1 as a Target for Melanoma Therapy--------------------------------------- 58

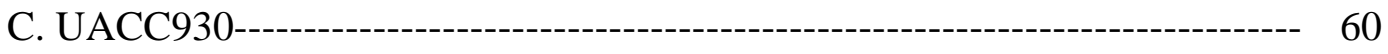

D. Glutamate Signaling as a Target for Melanoma Therapy---------------------- 61

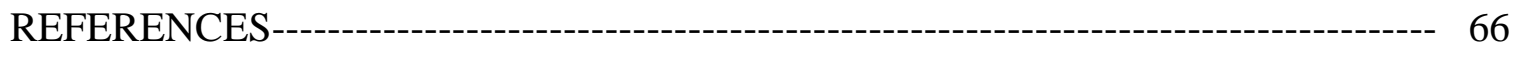

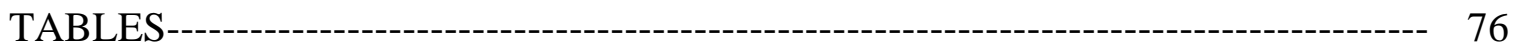


FIGURES------------------------------------------------------------------------------------------ 82

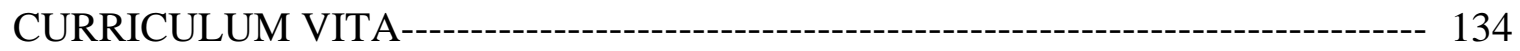




\section{LIST OF TABLES}

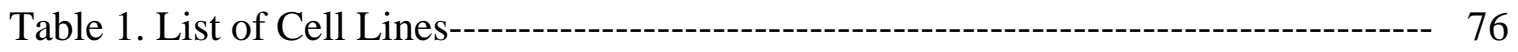

Table 2. Cell Cycle Analysis: 24 Hours After BAY 36-7620 Treatment of C8161----- 77

Table 3. Cell Cycle Analysis: 48 Hours After BAY 36-7620 Treatment of C8161----- 77

Table 4. Cell Cycle Analysis: 24 Hours After Riluzole Treatment of C8161------------ 78

Table 5. Cell Cycle Analysis: 48 Hours After Riluzole Treatment of C8161------------ 78

Table 6. Cell Cycle Analysis: 24 Hours After BAY 36-7620 Treatment of WM239A- 79

Table 7. Cell Cycle Analysis: 24 Hours After Riluzole Treatment of WM239A-------- 79

Table 8. Cell Cycle Analysis: 24 Hours After BAY 36-7620 Treatment of UACC903- 80

Table 9. Cell Cycle Analysis: 48 Hours After BAY 36-7620 Treatment of UACC903- 80

Table 10. Cell Cycle Analysis: 24 Hours After Riluzole Treatment of UACC903------ 81

Table 11. Cell Cycle Analysis: 24 Hours After Riluzole Treatment of UACC903------ 81 


\section{LIST OF FIGURES}

Figure 1. Grm1 Expression in TG-3 Mouse Tumors------------------------------------ 82

Figure 2. Histopathology of Tail Sections of Normal and E-line Mice------------------ 83

Figure 3. Activation/Inhibition of Grm1 by Agonist (Quisqualate) and Antagonist

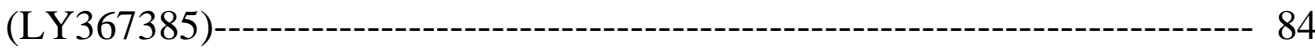

Figure 4. Grm1 Signaling Pathway in Melanoma----------------------------------------- 85

Figure 5. GRM1 Expression in Human Melanoma Biopsy Samples: RT-PCR--------- 86

Figure 6. GRM1 Expression in Human Melanoma Biopsy Samples: Q-PCR---------- 87

Figure 7. GRM1 Expression in Human Melanoma Biopsy Samples: Western Blots-- 88

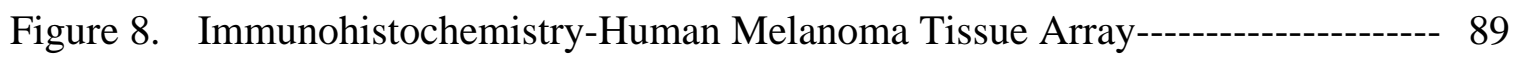

Figure 9. GRM1 Expression in Human Melanoma Cell Lines: RT-PCR---------------- 90

Figure 10. GRM1 Expression in Human Melanoma Cell Lines: Western Blot---------- 91

Figure 11. GRM1 Expression in Human Melanoma Cell Lines: Immunofluorescence- 92

Figure 12. IP3 Accumulation in C8161 Human Melanoma Cell Line-------------------- 93

Figure 13. IP3 Accumulation in WM239A Human Melanoma Cell Line---------------- 94

Figure 14. Activation/Inhibition of GRM1 by Agonist (Quisqualate) and Antagonist

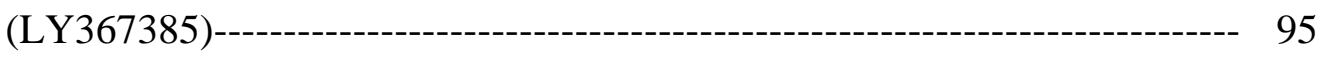

Figure 15. Apoptosis Induction by Dominant Negative Mutants of GRM1 (dnGRM1) in

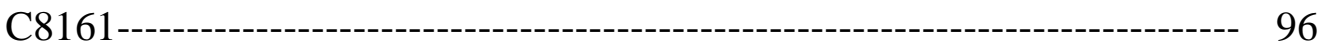

Figure 16. Apoptosis Induction by Dominant Negative Mutants of GRM1 (dnGRM1) in

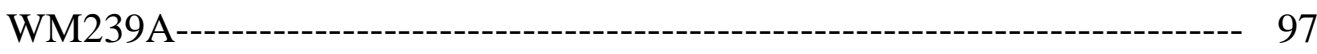


Figure 17. MTT Cell Proliferation Assay of C8161 and HEK293A Cells Treated with a

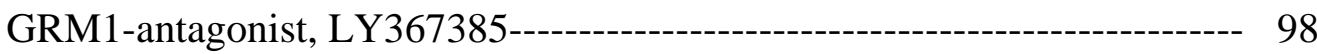

Figure 18. Suppression of ERK Activation by LY367385 Treatment--------------------- 99

Figure 19. MTT Cell Proliferation Assay of WM239A Treated with LY367385------- 100

Figure 20. MTT Cell Proliferation Assay of UACC903 Treated with LY367385------ 101

Figure 21. MTT Cell Proliferation Assay of WM115 Treated with LY367385--------- 102

Figure 22. MTT Cell Proliferation Assay of C8161 and HEM Cells Treated with a

GRM1- antagonist, BAY 36-7620------------------------------------------------ 103

Figure 23. MTT Cell Proliferation Assay of WM239A Treated with BAY 36-7620-- 104

Figure 24. MTT Cell Proliferation Assay of UACC903 Treated with BAY 36-7620-- 105

Figure 25. Cell Cycle Analysis of BAY 36-7620 treated C8161------------------------- 106

Figure 26. Cell Cycle Analysis of BAY 36-7620 treated WM239A---------------------- 107

Figure 27. Cell Cycle Analysis of BAY 36-7620 treated UACC903-------------------- 108

Figure 28. Induction of Apoptosis by BAY 36-7620 Treatments in C8161------------- 109

Figure 29. Grm1 siRNA Stable Clones----------------------------------------------------------- 110

Figure 30. Western Blots of C8161-GRM1 siRNA Stable Clones------------------------- 111

Figure 31. C8161-GRM1 siRNA Stable Clones: MTT Cell Proliferation Assay------- 112

Figure 32. Glutamate Release Measurements of Human Melanoma Cell Lines-------- 113

Figure 33. Glutamate Release Measurements of LY367385 treated C8161------------- 115

Figure 34. Glutamate Release Measurements of LY367385 treated HEK293A-------- 116

Figure 35. Glutamate Release Measurements of BAY 36-7620 treated C8161-------- 117

Figure 36. Glutamate Release Measurements of Riluzole treated C8161--------------- 118 
Figure 37. MTT Cell Proliferation Assay with the Glutamate Release Inhibitor, Riluzole

Figure 38. MTT Cell Proliferation Assay of Riluzole treated WM239A---------------- 120

Figure 39. MTT Cell Proliferation Assay of Riluzole treated UACC903--------------- 121

Figure 40. Cell Cycle Analysis of Riluzole treated C8161--------------------------------- 122

Figure 41. Cell Cycle Analysis of Riluzole treated WM239A----------------------------- 123

Figure 42. Cell Cycle Analysis of Riluzole treated UACC903---------------------------- 124

Figure 43. Induction of Apoptosis by Riluzole Treatments-------------------------------- 125

Figure 44. C8161 Xenograft Model for Riluzole IV Treatment--------------------------- 126

Figure 45. C8161 Xenograft Model for Riluzole Oral Treatment------------------------ 127

Figure 46. Immunohistochemistry: C8161 Xenograft Model for Riluzole Treatment- 128

Figure 47. UACC930-Grm1 Stable Clones: Western---------------------------------------- 129

Figure 48. UACC930-Grm1 Stable Clones: MTT Cell Proliferation Assay------------ 130

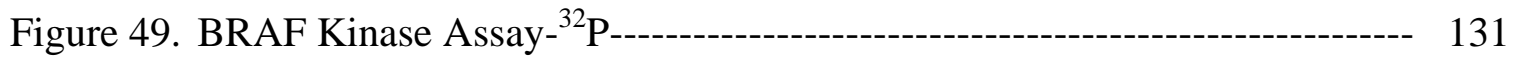

Figure 50. BRAF Kinase Assay-Western Blot----------------------------------------------- 132

Figure 51. Proposed GRM1 Signaling in Human Melanoma ---------------------------- 133 


\section{INTRODUCTION}

\section{PART I: SKIN CANCER}

\section{A. Three Types of Skin Cancer}

Skin cancers are the most common type of cancer. There are three major types of skin cancer: basal cell carcinoma, squamous cell carcinoma and melanoma. Squamous cell carcinoma and basal cell carcinoma together account for over one million cases each year in the United States, making them cancers with the highest incidence. Basal cell carcinoma is the most common type of cancer with over 800,000 people estimated to be diagnosed each year [1]. It originates from basal cells, keratinocytes located at the basal layer of epidermis. The aggressive form of basal cell carcinoma spreads to the surrounding tissues such as bones and cartilage [1]. However, basal cell carcinoma has low rates of metastasis and is not generally life-threatening. One cause of this cancer is sun exposure. Squamous cell carcinoma is the second most common skin cancer with approximately 200,000 incidences per year [2]. Squamous cells are epithelial cells that make up most of the skin. Most squamous cell carcinomas stay localized, however a small percentage of them metastasize leading to fatal conclusion [2]. A risk factor of squamous cell carcinoma is chronic sun exposure. Melanoma is the most malignant form of skin cancers. They originate from melanocytes, the pigment cells in the skin. Although only $5 \%$ of all skin cancers are melanoma, approximately $75 \%$ of deaths among skin cancer patients are attributed to this disease [3]. Melanoma will be discussed in more detail in the next section. 
Both basal cell carcinoma and squamous cell carcinoma are linked to overexposure to the sun. Ultraviolet (UV) rays induce the development of DNA adducts that are the initiation, or the early event in the skin carcinogenesis [4]. The presence of UVB-light-induced signature mutations, resulted from pyrimidine dimer formation, in the p53 tumor suppressor gene support the idea that the UV rays are the cause of these carcinomas [4]. Approximately half of sporadic basal cell carcinomas contain a mutation in the p53 tumor suppressor gene with UVB signature mutations [1]. Treatment options for both basal cell carcinoma and squamous cell carcinoma include surgical, such as curettage, electrodesiccation, cryosurgery, or surgical excision, and non-surgical methods, such as radiation for those locations that are difficult to treat $[1,4]$.

After initial tumor development, the patient relative risk for subsequent basal cell carcinoma increases 10-fold when compared to the general population [1]. Even though recurrence is uncommon, due to an increase in the relative risk of developing cancers, patients are recommended to undergo regular check-ups. In addition to a p53 mutation in $50 \%$ of basal cell carcinoma cases, deregulation of the hedgehog signaling pathway, a critical developmental signaling pathway, also has been linked to the incidence of basal cell carcinoma. Loss of function mutations of the patched homologue 1 (PTCH1), another tumor suppressor gene, has been found in $30-40 \%$ of basal cell carcinoma. PTCH1 interacts with a transmembrane protein similarly structured to a G-protein coupled receptor, smoothened (SMO) $[1,5]$. In the absence of PTCH1 repression, $\mathrm{SMO}$ is constitutively activated leading to the inhibition of the downstream targets of gene 
expression by the GLI family of transcription factors, which play critical roles in development and cancer cell proliferation $[1,5]$.

A different signaling pathway has been identified to be involved in squamous cell carcinoma. Studies identified activator protein-1 (AP1) and cyclooxygenase-2 (COX2) to be involved in carcinogenesis. UVB activates AP1 in keratinocytes, mediated by p38 and extracellular-signal regulated kinases (ERK), both of which are members of mitogenactivated protein kinases (MAPKs) [4]. MAPK activation leads to the activation of cyclic AMP response element binding protein (CREB), which in turn binds to the promoter region of c-FOS; and c-FOS activates AP1. CREB also activates COX2 transcription [4]. Even though squamous cell carcinoma has lower incidence rate, it is more dangerous than basal cell carcinoma with higher metastatic potential. The 5-year metastatic rate of squamous cell carcinoma is $5 \%$ [2].

\section{B. Melanoma}

Melanoma, the most dangerous form of skin cancer, is characterized by uncontrolled growth of melanocytes. Melanocytes are pigment cells that reside at the basal layer of the epidermis and produce melanin to protect the skin from UV damage. In the year 2007, approximately 60,000 new cases of melanoma were expected with over 8,000 deaths in the United States [3]. The overall lifetime risk of developing melanoma is one in 73 for women, and one in 49 for men. Furthermore, melanoma is one of the most common cancers among people under the age of forty [3]. There are four major types of melanoma: superficial spreading, nodular, lentigo maligna, and acral, as well as less usual 
types such as ocular and mucosal [6]. Different types of melanoma are likely due to differences in molecular mechanisms underlying the onset of the disease; the most common type is superficial spreading melanoma, which accounts for $80 \%$ of all melanomas [6]. Studies on the causes of melanoma have led to the identification of several risk factors, including the presence of congenital or dysplastic nevi, genetics (light skin, light hair color, poor tanning ability, etc.), UV radiation exposure, family history, and immunosuppression [3]. UV radiation exposure as a factor in melanoma development remains a center of controversy, as mechanism(s) have not been elucidated [7]. Development of melanoma is most likely due to the combination of several factors, including the tumor microenvironment [7].

Physicians generally are guided by the ABCD rule, which is Asymmetry, Border irregularity, Color variance, and Diameter [8], in examining suspicious moles. In addition, efforts to educate individuals about the dangers of skin cancer, risk factors and warning signs, have been important in assisting physicians to detect, diagnose and treat melanoma early, thereby increasing the overall survival of patients. Patients with localized tumors have a 5-year survival rate of 99\% [3]. However, if distant metastasis occurs, the 5-year survival rate falls to $15.3 \%$ [3]. Histopathology of the tumor determines the prognosis for the patient. Some prognostic markers for poor survival rates include thickness, ulceration, location of melanoma, lymph node involvement, and poor overall health status of the patient [8]. Melanoma thicker than $4 \mathrm{~mm}$ in diameter is associated with nodal and distant metastasis [8]. In the early 1990s, a new surgical technique known as sentinel node biopsy emerged [9]. This method permits the 
identification and removal of the particular lymph nodes to which tumors drain. Currently, this technique remains the most accurate staging indicator for melanoma [10]. Comparison between those patients who received sentinel node biopsy, and those in the "wait and watch" group, showed that the sentinel-node group had a $26 \%$ less chance of recurrence of melanoma [11]. 


\section{PART II: CURRENT TREATMENT OPTIONS}

\section{A. Interferon alpha}

The most effective treatment option for melanoma is surgical resection. Patients with tumors thicker than $4 \mathrm{~mm}$, or with positive sentinel node indicating infiltration of tumor cells, have medium to high risk for recurrent or metastatic melanoma. It is recommended that these patients undergo systemic adjuvant therapy after removal of primary tumors. The only adjuvant therapy approved by the Food and Drug Administration (FDA) for melanoma with high risk of recurrence, is interferon alpha [8]. Interferons, of which there are several types, are naturally produced by cells to protect themselves from viral infections. Once bound to their receptors, interferons activate downstream signaling cascades. The classical pathway involved in interferon signaling is signal transducers and activators of transcription (STAT). STAT proteins are transcription factors that translocate to the nucleus when activated, and regulate expression of gene products including those that are involved in apoptosis and inhibition of cell growth [12]. Several studies pointed to the ability of interferon to inhibit tumor cell proliferation; therefore, it has been used as a therapeutic agent for several cancers including melanoma [12]. Interferon was initially advanced as an option for melanoma treatment due to its ability to inhibit growth of B16 murine melanoma cells implanted in mice [13]. It was observed that, in hematopoietic cells, long-term treatment with interferon alpha induces cell cycle arrest at $\mathrm{G}_{0} / \mathrm{G}_{1}$, and short-term treatment inhibits the activation of cell signaling pathways, including the MAPK signaling pathway [14]. Studies of several human melanoma cell lines showed that both interferons alpha and beta induced apoptosis 
through caspases, Fas/FasL, and TNF-alpha related apoptosis inducing ligand (TRAIL), with interferon beta being more efficient [15]. In addition to induction of apoptosis, interferon also inhibits angiogenesis [16].

Clinical trials of low or intermediate doses of interferon on melanoma patients demonstrated no survival benefits, although meta-analysis indicated a $13 \%$ reduction in the odds of recurrence in some cases with low-dose interferon treatment [17]. Several clinical trials were performed in Europe with maximum tolerated dose of interferon in melanoma patients. One trial by the Eastern Cooperative Oncology Group (ECOG) that used high-dose interferon alpha- $2 \mathrm{~b}$ administered intravenously and subcutaneously (E1684), resulted in prolonged relapse-free and overall survival [18]. This trial was followed by E1690, which included both high-dose and low-dose interferon treatments [19]. Although relapse-free 5 year survival was improved in the high-dose group, the 5 year overall survival was not improved, possibly due to confounding factors such as a high-number of crossover of patients from observation group to high-dose group [20]. Low-dose interferon treatment did not improve relapse-free survival or overall survival [20]. After updating disease-status and survival, several trials were pooled to analyze data. For trial E1684, after follow-up of 12.6 years, statistical significance was observed in relapse-free survival, but not in overall survival [20]. Taken together, the pooled data revealed that there are advantages for high-risk patients to be treated with interferon due to improvement in relapse-free survival, despite lack of significance for overall survival [20]. The significance of interferon treatments remains controversial due to yet another pooled analysis of nine trials that revealed no advantage in disease-free survival or 
overall survival [21]. The response-rate is not substantial enough to warrant high-dose interferon treatments, especially since such options produce extremely unpleasant side effects [22]. Side effects of interferon treatment include flu-like symptoms, fatigue, diarrhea, and depression, thus requiring careful monitoring by administering physicians [17]. Since the effectiveness of interferon adjuvant therapy on patients with intermediate to high-risk melanoma remains unclear, additional therapeutic options for better relapsefree and overall survival would benefit melanoma patients.

\section{B. Interleukin-2}

Interleukin-2 (IL-2) is an immune-modulating glycoprotein. One of the functions of IL-2 is the activation of immune cells such as natural killer cells. This activity has generated interest in the use of IL-2 in cancer immunotherapy [23]. Regression of metastatic tumors has been shown with IL-2 in renal cell carcinoma [24]. Clinical response is measured in terms of complete response, partial response, and no response. In general, complete response represents regression of tumors followed by no growth for 6 months; partial response represents $50 \%$ reduction of tumors; and no response means no change [25]. The first randomized clinical trial for intermediate or high risk melanoma patients was conducted with subcutaneous injection of low-dose IL-2 in combination with interferon alpha-2B [26]. An overall survival benefit was not found for patients who received the combination therapy, when compared to observation group, therefore this clinical trial was unsuccessful. Treatment of patients with high-dose IL-2 alone has been approved by the FDA for metastatic melanoma, although results have been disappointing with only $16 \%$ of patients showing a response [27]. Treatment with IL-2 causes severe side effects, 
including, but not limited to, hypotension, myocarditis, transient renal insufficiency, and capillary-leak syndrome [28]. There have been attempts to alter dose levels and change routes of administration in clinical trials, to diminish these severe side effects and to improve response-rate. Recently, intra-lesional injection of IL-2 was done in patients with skin and soft-tissue metastasis; $62.5 \%$ of patients had complete response and another $21 \%$ showed partial response [25]. With intra-lesionally delivered IL-2, patients tolerated this route of administration well with minimal side effects [25]. For melanoma patients with metastasis who did not respond to current therapeutic regimens, a new immunotherapy was tested. This immunotherapy consisted of chemotherapy, followed by re-introduction of the patient's own activated lymphocytes in combination with high-dose IL-2 adjuvant therapy [29]. About 51\% of the patients showed improvement, from complete responses $(8.6 \%)$ to partial responses $(43 \%)$ with manageable side effects.

\section{Others}

Compounds such as dacarbazine and cisplatin, among others, have been used in clinical trials for treatment of stage IV (metastatic) melanoma, in hopes of obtaining a better response-rate and prolonged survival of patients. Dacarbazine, an alkylating agent, showed the highest responses among selected chemotherapeutic drugs [30]. After combining data gathered through several clinical trials over the years, Eggermont and Kirkwood reported that dacarbazine alone showed similar or better responses, compared to when dacarbazine was used in combination with other drugs [30]. In a phase III clinical trial of stage IV melanoma patients, no differential survival benefit was shown 
between patients treated with dacarbazine alone, and patients treated with the Dartmouth regimen (dacarbazine, cisplatin, carmustine, and tamoxifen) [31].

In addition to the quest for novel and more effective drugs, many groups are actively pursuing improvements in gene therapy for the treatment of melanoma. The ideal way to correct a lost or defective gene would be to replace it, in order to restore proper cellular function. In melanoma, tumor suppressor genes such as PTEN, or cell cycle regulators such as $\mathrm{p} 16^{\mathrm{Ink} 4 \mathrm{~A}}$, have been found to be lost in many cases; therefore they represent good candidates for replacement by gene therapy. However, gene therapy has been proven to be a simple concept with numerous complex problems in targeting, delivery, dosage, toxicity, and immune responses [32]. The first successful gene therapy trial was with children suffering severe combined immunodeficiency (SCID); failing other treatments, such patients are required to stay within a germ-free environment. They develop SCID due to a defective cytokine receptor gamma chain, and replacement of the defective gene by gene therapy was touted as freeing children from their confinement. However, more recent reports indicate that some of the children so treated, later developed leukemia [33]. Another gene therapy trial resulted in the death of a patient with ornithine transcarbamylase deficiency, just a few days after treatment in 1999 [34]. This highly publicized case brought attention to the limitations of gene therapy, including the safety and toxicity of delivery vehicles. Despite such limited successes in treating patients with gene therapy, investigators continue to improve techniques, and to devise safer ways to deliver and replace gene(s) for effective restoration of their normal functions. For melanoma or other skin diseases, one of the major problems of gene therapy is proper 
targeting to the skin. One way to deliver genes to the skin is by electroporation [35]. Electroporation, which opens transient pathways into cells by means of short electric pulses, has been used to deliver exogenous DNA into human skin xenografts [35]. In that study, electroporation greatly enhanced delivery of DNA compared to intradermal injection of DNA alone. In addition, the expression of a given gene was sustained for longer than 15 days, which was the maximum time point measured. This new technical improvement increases the feasibility for the future use of gene therapy for the treatment of melanoma by delivering active reagents to the skin.

Despite numerous uncertainties underlying the mechanisms of action, and the recurrence and persistence of metastases after treatments with interferon alpha and IL-2, these two options continue to be the most promising management techniques for overall survival of melanoma. Taken together, the necessity to discover novel and more relevant therapeutic targets remains a top priority. Based on published literature and results from our laboratory, metabotropic glutamate receptor 1 and glutamate signaling pathway are plausible targets of new melanoma therapy. 


\section{PART III. METABOTROPIC GLUTAMATE RECEPTOR 1 (GRM1)}

\section{A. Mouse Model for Melanoma}

Animal models have evolved as indispensable tools to study the initiation, progression, and treatments of many different types of human disease. Animal model systems also provide means for understanding the molecular mechanisms underlying a particular disorder and provide opportunities to identify putative targets, and discover and develop new drugs and treatments. In the last 15 years, an increasing number of animal models have been developed and used to study a diverse array of cancers including melanoma. Our laboratory has developed a transgenic mouse model (TG-3) that is predisposed to spontaneous melanoma development. Onset of melanoma in TG-3 occurs with high penetrance, short latency and high metastatic potential [36-38]. Spontaneous development of melanoma and tumor progression in TG-3 mimics and recapitulates the evolution of melanoma as seen in humans, from benign lesions to metastasis [36, 37]. In TG-3, we showed that insertional mutagenesis by a transgene resulted in the appearance of pigmented lesions which subsequently were confirmed to be melanoma by histopathology [38, 39]. Extensive molecular studies revealed that the transgene had integrated into intron 3 of metabotropic glutamate receptor 1 (Grm1), resulting in ectopic expression of Grm1 in melanocytes and leading to tumor development [40]. Grm1 expression was examined in tumor ear samples and in normal ear samples by duplex reverse transcriptase polymerase chain reaction (RT-PCR) in Figure 1. Tyrosinase related protein 1 (Tyrp1) and Grm1 primers were used to control for the different number of melanocytes present in normal ear and tumors. Similar level of Tyrp1 expression was 
detected in all ear samples; however Grm1 expression was detected only in tumor ears. In order to distinguish whether the aberrant expression of Grm1 in tumors was a cause or a consequence of melanoma, a new transgenic line (E line) was constructed. It was engineered to express Grm1 in melanocytes by placing cDNA under the regulation of a melanocyte-specific promoter, dopachrome tautomerase (DCT). E line developed melanoma as demonstrated by histopathology in Figure 2. This new E transgenic line displayed similar onset and progression of melanoma as observed for TG-3. Taken together, results from these studies demonstrated that aberrant expression of Grm1 in melanocytes is sufficient to induce melanoma development in vivo [40].

\section{B. Grm1 Signaling}

Grm1 is a seven-transmembrane-domain, G-protein-coupled receptor (GPCR), normally expressed and functional in the central nervous system. The natural ligand for Grm1 is glutamate, a major neurotransmitter. To date, eight metabotropic glutamate receptors have been discovered and they are classified into three groups according to sequence homology and pharmacological profiles. Grm1 and Grm5 belong to group I metabotropic glutamate receptors. Group I receptors, when stimulated, are preferentially coupled to $\mathrm{G}_{\mathrm{q} / 11}$ leading to phospholipase $\mathrm{C}$ (PLC) activation, and give rise to two second messengers: diacylglycerol (DAG) and inositol-1, 4, 5-triphosphate (IP3). DAG, in turn, activates protein kinase $\mathrm{C}$ (PKC) and IP3, leading to $\mathrm{Ca}^{2+}$ release from the endoplasmic reticulum, the intracellular calcium storage. Involvement of GPCR signaling in tumor development, especially in melanoma, was reviewed recently [41]. GPCRs such as endothelin receptor (ETRB) have been implicated to be important in tumor cell 
proliferation [41]. A specific inhibitor to ETRB, BQ788, induced apoptosis in melanoma cells in vitro and growth arrest in xenografts of human melanoma cells in nude mice [42]. Cell signaling mediated by Grm1 has been extensively studied in neuronal cells, as well as in heterologous cells transfected with Grm1. In the heterologous systems, activation of Grm1 by its natural ligand glutamate, or its agonist L-quisqualate, leads to activation of extracellular-signal regulated kinases (ERK) [43, 44]. This activation is inhibited by PD 098059, a MAPK/ERK kinase (MEK) inhibitor, implicating involvement of MEK/ERK in Grm1 signaling [43].

Activation of MAPK pathway has been demonstrated to be an important event in human melanoma. Constitutively activated MAPK has been detected in several human melanoma cell lines and biopsies [45]. In our laboratory, several TG-3 tumor-derived cell lines were established. We showed these cells to be transformed and tumorigenic [46]. We confirmed Grm1 in these cells to be functional as demonstrated by the accumulation of IP3 in the presence of Grm1-agonist. Furthermore, we also showed the activation of ERK by Grm1-agonist as shown in Figure 3 [47]. The specificity of Grm1 signaling in IP3 accumulation and activation of ERK was demonstrated by suppression of both activations by Grm1-specific antagonist, LY367385 [47]. Additionally, dominantnegative mutants of Grm1 [48] were used to further verify Grm1 signaling in these mouse melanoma cells [47]. The Grm1 signaling pathway in melanoma, deduced from our studies, is shown in Figure 4. 
There are several pharmacological agents available to block Grm1 signaling. These antagonists have been shown to be valuable in elucidating the physiological functions of Grm1 in neuronal cells. LY367385 [(+)-2-methyl-4-carboxyphenylglycine] is a specific competitive antagonist of Grm1 [49]; CPCCOEt [(-)-(1aS, 7aS)-(2-hydroxyimino-1a, 2dihydro-1H-7-oxa-cyclopropa[b]naphthalene-7a-carboxylic acid ethyl ester) is a noncompetitive antagonist [50]; and BAY 36-7620 [(3aS, 6aS)-6a-naphtalen-2-ylmethyl5-methyliden-hexahydro-cyclopental[c]furan-1-on] is also a noncompetitive antagonist of Grm1 with inverse agonist activity [51]. Inverse agonists are antagonists that inhibit both agonist-induced activation and constitutive activation of receptors. Overexpression of group I metabotropic glutamate receptors (Grm1 and Grm5) resulted in constitutive activation of receptors in heterologous systems [52]. However, the constitutive activation of Grm1 is inhibited by BAY 36-7620, an inverse agonist [53, 54]. Based on these earlier studies, we hypothesize that constitutive activation of Grm1 may be required for the maintenance of melanocyte transformation and melanoma development. Recent studies showed that a noncompetitive antagonist with inverse agonist activity, such as BAY 367620, can inhibit both agonist-induced and constitutive activity of Grm1 $[53,54]$, thus BAY 36-7620 may be useful for suppression of tumor progression. 


\section{PART IV. MELANOMA CELL PROLIFERATION PATHWAY AND IT'S INHIBITORS}

The MAPK pathway has been shown by others to be one of the most important pathways in melanoma cell proliferation $[45,55]$. Our laboratory has potentially identified MAPK as one of the pathways activated in response to Grm1 stimulation; however molecules that participate in Grm1-to-ERK cascade as well as downstream targets of activated ERK remain to be identified. As stated earlier, results from our studies showed that treatment of TG-3 tumor cells with Grm1-agonist, L-quisqualate, resulted in activation of MEK and ERK [47], which are two of the integral components of the RAS/RAF/MEK/ERK pathway.

\section{A. RAS}

RAS is a membrane-bound G-protein that conveys a wide variety of extracellular signals to specific intracellular signaling pathways. There are three major isoforms of RAS: KRAS, NRAS and HRAS. Upon activation by extracellular signals, RAS-specific guanine-nucleotide-exchange factors (GEFs) promote the exchange of GDP to GTP, activating RAS. RAS has been demonstrated to link receptor tyrosine kinases such as the epidermal growth factor receptor (EGFR) to downstream effectors such as RAF (MEKK) through Grb2, an adaptor protein, and son of sevenless (SOS), a guanine nucleotide exchange factor [56]. Several mutants of RAS, which lack GTP hydrolase activity, have been shown to be most effective in promoting RAS-dependent signaling [56]. Depending on cell type or receptors involved, RAS-dependent signaling pathways may be important 
in mediating cell differentiation or proliferation [57]. MAPK, specifically the RAF/MEK/ERK cascade, is one of the downstream targets of activated RAS that leads to cell proliferation [56].

In human melanoma, about $15 \%$ of tumors and cell lines were reported to carry NRAS mutations [58]. Previous studies showed the involvement of an activating mutant of NRAS in promoting melanoma cell proliferation; therefore, mutated NRAS has been recognized as a prime target for melanoma therapy. RAS is only active when it is bound to the plasma membrane. This is achieved through prenylation, in particular farnesylation, an addition of a 15-carbon group. Prevention of RAS signaling has been accomplished through inhibitors of farnesylation, blocking the membrane localization of RAS and interfering with oncogenic RAS signaling cascade [56]. For example, an inhibitor of farnesyl transferase, SCH66336, inhibited B16 melanoma cell growth by inducing cell cycle arrest at G1 and inactivating the retinoblastoma (RB) protein [55]. Cisplatin is a DNA damaging agent that induces apoptosis in cancer cells [59]; however some melanoma cells have been shown to be resistant to cisplatin. When used in combination with SCH66336, enhanced cisplatin-induced apoptosis was detected in melanoma cells [55]. Since the actions of farnesylation inhibitors are not restricted to RAS, additional studies are needed to address general cytotoxic effects. Farnesyl thiosalicylic acid is a RAS antagonist that inhibits attachment of RAS to membrane, thereby promoting degradation of RAS. It has been shown to have anti-tumor activities by inducing apoptosis of Colo853 and B16 melanoma cells [60, 61]. Farnesyl thiosalicylic acid thus may be a better therapeutic agent than farnesyl transferase 
inhibitors, due to its higher specificity for RAS and lower general cytotoxicity [61]. As an alternative, inhibition of constitutive RAS signaling may also be accomplished by the use of antisense oligonucleotides or RNA interference (RNAi). Suppression of mutant protein expression via either method can be achieved with higher specificity than by pharmacological agents. Mutation of NRAS at codon 61 is the most frequent RAS activating-mutation detected in human melanomas [56]. Small interfering RNA (siRNA), targeted to the Q61R activating mutation in human melanoma cells, resulted in both suppression of downstream activation of ERK and induction of apoptosis [62].

\section{B. RAF}

RAF protein is one of the downstream targets of RAS. In general, activated RAF phosphorylates MEK, and activated MEK, in turn, phosphorylates ERK. There are three RAF isoforms: ARAF, BRAF and CRAF. CRAF, also known as RAF-1, is ubiquitously expressed in all tissues. ARAF is highly expressed in ovary, but also detected in several other tissues at varying levels. However, BRAF expression is restricted mostly to neuronal tissues [63]. Transforming activity of RAS was impaired in a heterologous system (COS-M6) by dominant-negative mutants of CRAF [64]. Although CRAF has been extensively studied, the regulation of RAF proteins, redundancy of their functions, and interactions among different RAF isoforms, generally are not well understood [65]. In melanoma cells, depletion of ARAF and CRAF by siRNA did not inhibit the activation of MEK and ERK, while depletion of BRAF by siRNA inhibited MEK and ERK phosphorylation [66, 67]. On the other hand, in human melanoma cells that harbor activating RAS mutations, or in RAS-transformed mouse melanocytes, activations of 
MEK and ERK were not affected by depletion of either BRAF or CRAF [67]. These observations suggest RAS signaling may be mediated through molecules other than BRAF or CRAF, or of functional redundancy between BRAF and CRAF. A more recent report indicated that in human melanoma cells harboring NRAS (Q61R) mutation as well as deregulated cAMP signaling, reducing CRAF expression by siRNA, suppressed ERK activation while reducing BRAF expression had no effects [68]. Taken together, results from these studies showed that depletion of one of the RAF isoforms may not be sufficient to inhibit downstream targets either in RAS-transformed cells, or in cells harboring mutant RAS, and may require additional disruption(s) in signaling pathways.

The most common mutation of BRAF (V600E) has been detected in $66 \%$ of human melanomas [69]. Subsequent studies by Dong et al. also have found that approximately $70 \%$ of nevi contain BRAF mutations, while only $10 \%$ of radial growth phase (RGP) melanoma, or early stage melanoma, carried this BRAF mutation. These findings imply that perhaps most nevi with BRAF mutations may not progress to the next stage in melanoma development $[70,71]$, and that BRAF may be involved in the initiation rather than the progression of melanoma. However, others have reported higher rates of BRAF mutations in early as well as later stage melanomas. Goydos et al. showed that $55 \%$ of primary melanomas, $67 \%$ of regional metastases, $40 \%$ of nodal metastases, and $75 \%$ of distant metastases carry a detectable V600E mutation [72]. Nonetheless, BRAF is an important potential target for melanoma therapy since it is involved in a high percentage of melanomas, and also plays a pivotal role in the proliferation of melanoma cells. Normal melanocytes require supplementation with the phobol ester, TPA (12-o- 
tetradecanoyl phorbol-13-acetate), for growth; and loss of this requirement is one of the indicators of melanocyte transformation [73]. When normal mouse melanocytes (Melana) were transfected with mutated BRAF (V600E), stable clones selected no longer required the presence of TPA for growth [67]. Inhibition of this BRAF mutant by siRNA led to inhibition of MEK phosphorylation, while inhibition of CRAF did not [74]. Cyclin D1, a key protein in cell cycle progression was also shown to be a downstream target of activated MAPK. When mutant BRAF was transfected into normal human epidermal melanocytes, an increase in cyclin D1 expression was observed; melanoma cells which harbored mutant BRAF displayed constitutive activation of cyclin D1 [75]. Inhibition of the downstream target of BRAF, MEK, by U0126 abolished cyclin D1 expression, and direct targeting of cyclin D1 by siRNA was shown to inhibit DNA synthesis in mutant BRAF melanoma cells [75].

In related efforts to inhibit mutant BRAF using RNAi, conflicting reports have emerged. One group reported that siRNA specific for mutant BRAF induced apoptosis in a human melanoma cell line [74]; whereas another group suggested that apoptosis induced by inhibition of BRAF by siRNA is not a general response, but rather cell line-dependent [76]. In any case, both groups do agree that RNAi, successfully targeted to mutant BRAF, would provide an excellent therapeutic target due to its specificity and its inhibition of growth and invasion of melanoma cells.

BAY 43-9006 (Sorafenib) is a potent kinase inhibitor against both B- and CRAF proteins and vascular endothelial growth factor receptor (VEGFR2) among others. It is effective 
against both wild-type and mutant BRAFs. BAY 43-9006 was shown to inhibit the growth of melanoma cells in vitro and in vivo; however, no apoptosis was detected, implying the possible requirement of combination therapies for optimal use against tumor cells [66]. Clinical trials using BAY 43-9006 went on to assess the effectiveness of this compound in clinical setting [77]. Recently, discontinuation of Phase II randomized clinical trial in advanced melanoma patients was reported due to little or no antitumor activity [78]. Overall after 12-week of receiving BAY 43-9006, only 19\% of patients had stable disease while $62 \%$ of patients had progressive disease. Status of BRAF mutation did not have any relationship on the stability of disease in these patients. Even though BAY 43-9006 was not useful treating advanced melanoma patients, it was approved by FDA to treat advanced renal cell carcinoma due to better progression free survival [79]. Since BAY 43-9006 is a general multikinase inhibitor, more specific inhibitors to mutant BRAF are being actively pursued by several investigators for melanoma therapy.

The antibiotic geldanamycin displays potent anti-tumor activity; its mechanism of action was shown to mediate through inhibition of heat-shock protein 90 (Hsp90) [80]. Consequently, Hsp90 has emerged as a new molecular target for cancer treatment. Hsp90 is a chaperone involved in ensuring proper folding and conformational maintenance of proteins, properties which are critical for the function and stability of a given protein [81]. Client proteins of Hsp90 include wild-type and mutant BRAFs, which has been demonstrated by co-immunoprecipitation in extracts from BRAF transfected cells [82]. A derivative of geldanamycin, 17-(Allylamino)-17-demethoxygeldanamycin (17-AAG), has similar potency with less general toxicity, when compared to geldanamycin. 17-AAG has 
been shown to inhibit tumor growth in a human melanoma xenograft model [83], and it is currently in phase I/II clinical trials for melanoma [11].

\section{MEK/ERK}

U0126 and PD 098059 are specific inhibitors of MEK1 and MEK2, the downstream targets of activated RAF. Urokinase plasminogen activator (uPA), colleagenase-1 (MMP1), and matrix metalloproteinase-9 (MMP-9), are proteins known to be involved in tumor cell invasion and have been demonstrated to be elevated in melanoma cells $[84,85]$. Treatment of melanoma cells with either U0126 or PD 098059 results in a decrease in levels of uPA, MMP-1, and MMP-9 as well as inhibition of cell growth [85-87]. One group showed that pretreatment of cisplatin-resistant melanoma cells with the MEK inhibitor PD 098059, followed by cisplatin treatment, results in apoptosis [88]. These results imply that cisplatin might be more effective in treating melanoma when used in combination with drugs that inhibit cell growth without inducing apoptosis, such as SCH66336, the inhibitor of farnesyl transferase (see above).

MAPK pathway plays a pivotal role in mediating signal transduction cascades regulating cell proliferation, including the induction of transcription factors that promote cell growth. The potential of such transcription factors as targets for melanoma therapy has been the topic of intense study. Activation of BRAF in melanoma cells induces the expression of the transcription factor, Brn-2 [89]. Downregulation of Brn-2 by RNAi in human melanoma cells, results in inhibition of $\left[{ }^{3} \mathrm{H}\right]$-thymidine incorporation indicating a reduction in DNA synthesis [89]. Some other downstream targets of activated MAPK 
pathways have been identified; they include the cAMP response element binding protein (CREB). Overexpression of CREB and a related protein, activating transcription factor 1 (ATF-1), has been observed in melanoma. Based on results from earlier reports, using monoclonal antibodies to disrupt the activity of ATF-1, an anti-ATF-1 single chain antibody fragment was engineered and shown to effectively inhibit ATF-1 activity, leading to the suppression of both tumorigenicity and metastasis of human melanoma cells [90].

Glutamate signaling has been well characterized in neuronal cells where glutamate is the major neurotransmitter. However, its signaling is not well understood in other tissues. The first reported participation of aberrant metabotropic glutamate receptor signaling in cancer, was the involvement of metabotropic glutamate receptor 1 (Grm1) in melanoma $[37,40]$. More recently, another metabotropic receptor, metabotropic glutamate receptor 4, was reported to be overexpressed in colorectal cancer [91]. The anticancer potential of ionotropic glutamate receptor antagonists has been examined by others; in cancers such as breast, colon, astrocytoma and lung, treatment of tumor cells by antagonists of ionotropic glutamate receptors resulted in inhibition of tumor cell proliferation and decreases in tumor cell motility mediated through the MAPK signaling pathway [92]. These observations, as well as data from our mouse models, underscore the importance of glutamate signaling in tumor development and progression. It will be of significant interest to further explore the glutamate signaling as a therapeutic target of melanoma. 


\section{MATERIALS AND METHODS}

\section{A. Antibodies and Reagents}

Anti-phospho-ERK, anti-ERK, anti-phospho-MEK, anti-MEK and anti-PARP were purchased from Cell Signaling (Danvers, MA); Grm1 antibodies were purchased from BD Biosciences (Franklin Lakes, NJ) and ImmunoStar Inc. (Hudson, WI); anti-BRAF antibody was purchased from Upstate (Charlottesville, VA); monoclonal $\alpha$-tubulin antibody, myo-inositol and Riluzole were obtained from Sigma (St. Louis, MO). Dimethyl sulfoxide (DMSO) was purchased from Fisher Scientific (Pittsburgh, PA). Lquisqualate $[(\mathrm{L})-(+)$-a-Amino-3,5-dioxo-1,2,4-oxadiazolidine-2-propanoic acid] and LY367385 [(S)-(+)-a-Amino-4-carboxy-2-methylbenzeneacetic acid] were purchased from Tocris (Ellisville, MO). BAY 36-7620 [(3aS,6aS)-6a-Naphtalen-2-ylmethyl-5methyliden-hexahydro-cyclopental[c]furan-1-on] was obtained from Bayer (West Haven, CT).

\section{B. Cell Culture}

Primary human epidermal melanocytes (HEM) were purchased from Cascade Biologics (Portland, OR) and they were maintained in Medium 254 with human melanocyte growth supplements (HMGS) (Cascade Biologics). UACC930, UACC903 and A2058 were provided by Dr. Jeffrey Trent (The Translational Genomics Research Center, Phoenix, AZ). WM239A, WM115 and WM35 were from Dr. Meenhard Herlyn (the Wistar Institute, Philadelphia, PA). C8161 and C81-61 were from Dr. Mary J. C. Hendrix (Children's Memorial Research Center, Chicago, IL). Melanoma cells were grown in 
RPMI 1640 plus 10\% FBS. For glutamate measurement or induction experiments with GRM1-agonist, in order to minimize glutamate in the media, customized glutamine- and glutamate-free RPMI media (Invitrogen-Gibco, Carlsbad, CA) was used with 10\% dialyzed FBS (Invitrogen-Gibco) and supplemented with 2 mM GlutaMax (InvitrogenGibco). For the measurement of inositol-1,4,5-triphosphate (IP3), customized glutamineand glutamate-free RPMI media were additionally deprived of inositol (InvitrogenGibco).

\section{Immunohistochemistry-ApopTag}

ApopTag In Situ Apoptosis Detection Kit (Upstate-Chemicon, Temecula, CA) was used to detect apoptotic cells in Riluzole-treated, or control tissue sections. This kit utilizes terminal deoxynucleotidyl transferase (TdT), which adds nucleotide triphosphates to free 3'OH end in DNA termini. These nucleotide triphosphates form an oligomer with digoxigenin-conjugated nucleotides. Anti-Digoxigenin peroxidase antibody binds to digoxigenin and color develops from peroxidase substrate, to show apoptotic cells. First, tissue sections were deparaffinized by three washes with xylene, 5 minutes each. Then slides were placed in 100\% ethanol (5 min), 100\% ethanol (5 min), 95\% ethanol (3 min) and $70 \%$ ethanol $(3 \mathrm{~min})$ followed by 5 minutes of PBS wash. Proteinase K $(20 \mu \mathrm{g} / \mathrm{mL})$ was added to slides for 15 minutes at room temperature for antigen retrieval. Slides were washed twice with $\mathrm{dH}_{2} \mathrm{O}$ for 2 minutes each. Endogenous peroxidase was quenched using $3 \% \mathrm{H}_{2} \mathrm{O}_{2}$ in PBS for 5 minutes, followed by two washes using $\mathrm{dH}_{2} \mathrm{O}$ for 5 minutes each. Equilibration buffer supplied by the kit was applied to slides for 2 minutes followed by addition of $30 \%$ TdT enzyme in reaction buffer supplied by the kit. Slides were incubated 
for one hour in a humidified chamber at $37^{\circ} \mathrm{C}$. Working concentration of Stop/Wash buffer was added to slides for 10 minutes, followed by three washes with PBS for 1 minute each. Anti-digoxignenin peroxidase was added and incubated for 30 minutes at room temperature in a humidified chamber, followed by four washes of PBS for 2 minutes each. Diluted peroxidase substrate was added for 3 to 6 minutes at room temperature and washed three times with $\mathrm{dH}_{2} \mathrm{O}$ for 1 minute each followed by incubation in $\mathrm{dH}_{2} \mathrm{O}$ for 5 minutes. Slides were incubated in Mayer's hematoxylin for 15 minutes for counterstain followed by running $\mathrm{dH}_{2} \mathrm{O}$ wash for 20 minutes. Specimen was mounted using VectaMount AQ (Vector Laboratories, Inc., Burlingame, CA) and sealed. Apoptotic cells were viewed using a Zeiss microscope.

\section{Immunocytochemistry}

Cells were plated on glass coverslips in $60 \mathrm{~mm}$ tissue culture plates. When cells reached approximately $70 \%$ confluence, they were fixed in cold methanol/acetone (1:1) for 5 minutes, rinsed twice with Phosphate Buffered Saline (PBS) or they were fixed in 90\% methanol followed by PBS rinse. Cells were first blocked with bovine serum albumin (BSA) for 30 minutes at room temperature. Coverslips were washed twice with PBS. Primary antibody incubation was for 45 minutes at room temperature with dilution factor suggested by the manufacturer. Rabbit anti-GRM1 (Abcam, Cambridge, MA) was diluted to $5 \mu \mathrm{g} / \mathrm{ml}$ and goat anti-Tyrp1 (Santa Cruz Biotechnology, Santa Cruz, CA) was diluted to $5 \mu \mathrm{g} / \mathrm{ml}$. After incubations, coverslips were washed twice in PBS. Cy3-conjugated anti-rabbit antibody ( $5 \mu \mathrm{g} / \mathrm{ml}$, Jackson ImmunoResearch Laboratories, Inc., West Grove, PA) or AlexaFluor 488 dye-conjugated anti-goat antibody $(7.5 \mu \mathrm{g} / \mathrm{ml}$, Molecular Probes, 
Eugene, OR) was used as the secondary antibodies. The secondary antibodies were incubated for 30 minutes at room temperature protected from light. Coverslips were washed with PBS and mounted. Fluorescent cells were viewed using a Zeiss microscope.

\section{E. Protein, Western Immunoblots and RNA Extraction}

Human tissues were obtained from Dr. James S. Goydos at the Cancer Institute of New Jersey (CINJ). Tissues were crushed by a mortar and a pestle in liquid nitrogen. Ground tissues were divided into two $14 \mathrm{~mL}$ snap cap Falcon tubes. For protein extraction, the extraction buffer consisted of 50mM Tris-HCL, 150mM NaCl, 1mM EDTA, 1\% NP40, 5\% Glycerol, 1mM Dithiothreitol, Complete protease inhibitor cocktail (Roche, Basel, Switzerland), and phosphatase inhibitors I and II (Sigma). Extraction buffer was added to tissue samples and homogenized using a Polytron (Brinkmann Instruments, Westbury, NY). Tissue was further lysed by rocking for one hour at $4^{\circ} \mathrm{C}$. For protein extraction from cell culture samples, media was removed and cells were washed once with ice-cold phosphate buffered saline (PBS). After removal of PBS, the extraction buffer was added directly to the plates and cells were collected with a cell scraper. Cells were incubated on ice for 20 minutes. Cell debris was collected from both tissue and cell culture samples by centrifugation at $10,000 \times \mathrm{g}$ at $4^{\circ} \mathrm{C}$ for 20 minutes. Protein lysates were collected into a fresh Eppindorf tube and protein concentration was determined using a DC protein assay kit (Bio-Rad, Hercules, CA). Routinely, $25 \mu \mathrm{g}$ of protein lysates were loaded for western immunoblots. 
For RNA extraction, TRI reagent LS (Molecular Research Center, Cincinnati, OH) was added to ground tissues and homogenized by a Polytron. For cells in culture, media was removed and $0.75 \mathrm{~mL}$ TRI reagent LS was added per $5 \times 10^{6}$ cells. Cells were collected using a cell scraper and suspended by passing through a pipette several times. $0.1 \mathrm{~mL} \mathrm{1-}$ bromo-3-chloropropane (BCP) per $0.75 \mathrm{~mL}$ TRI reagent, was used to separate phases. Samples were centrifuged at $12,000 \mathrm{x}$ g for 3 hours (tissue) or 15 minutes (cells) at $4^{\circ} \mathrm{C}$. The aqueous, colorless upper phase which contained RNA was collected into a fresh tube and $0.5 \mathrm{~mL}$ isopropanol per $0.75 \mathrm{~mL}$ TRI reagent was added to precipitate RNA. Precipitated RNA was cetrifuged at $12000 \mathrm{x} \mathrm{g}$ for 15 minutes (Tissue) or 8 minutes (Cells) at $4^{\circ} \mathrm{C}$ and the RNA pellet was washed with $1 \mathrm{~mL}$ of RNase-free $75 \%$ ethanol. Washed samples were spun down at $7500 \mathrm{x}$ g for 5 minutes at $4^{\circ} \mathrm{C}$ and the pellet was airdried. The pellet was resuspended with RNase-free water and the integrity of the samples was visualized by the presence of $18 \mathrm{~S}$ and $28 \mathrm{~S}$ ribosomal RNA in an agarose gel. $2-5 \mu \mathrm{g}$ RNA was used to synthesize cDNA using SuperScript II (Invitrogen) according to the manufacturer's instructions.

\section{F. Inositol-1,4,5-triphosphate Measurements}

IP3 was measured as described previously with minor modifications [44, 47]. Cells were plated at $10^{4}$ cells per well in RPMI with $10 \%$ FBS in 24 well plates. Media were changed sequentially every 24 hours. The growth media was changed to glutamate-free RPMI with 10\% dialyzed FBS, then glutamate/inositol-free RPMI with 10\% dialyzed FBS and finally to glutamate/inositol/serum-free RPMI in the presence of $3 \mu \mathrm{Ci}$ of myo$\left[{ }^{3} \mathrm{H}\right]$-inositol (3.22 TBq/mmol, GE Healthcare, Piscataway, NJ). After 18 hours, cells 
were incubated in fresh glutamate/inositol/serum-free RPMI with $\mathrm{LiCl}(10 \mathrm{mM})$ for 15 minutes in the presence or absence of LY367385 $(10 \mu \mathrm{M})$ prior to stimulation with Lquisqualate $(10 \mu \mathrm{M})$ for 15 minutes. The reactions were terminated by scraping cells into $500 \mu \mathrm{L}$ of $1 \mathrm{M}$ trichloroacetic acid (Sigma) followed by the addition of $100 \mu \mathrm{L}$ of $10 \mathrm{mM}$ EDTA (pH 7.0). Samples were either washed with the addition of $1 \mathrm{~mL}$ of a 1:1 mixture of 1,1,2-trichlorotrifluoroethane (Sigma) and tri-n-octalamine (Sigma) or washed twice with water-saturated diethyl ether (Sigma). The samples were then transferred to tubes containing $100 \mu \mathrm{L}$ of $62.5 \mathrm{mM} \mathrm{NaHCO}$. Formate columns were prepared by stuffing 3 $\mathrm{mL}$ syringes with glass wool and Dowex AG 1-X8 ion-exchange formate resin (BioRad). $1 \mathrm{~g}$ of Dowex AG 1-X8 ion-exchange formate resin was hydrated with $8 \mathrm{~mL} \mathrm{H}_{2} \mathrm{O}$ for 5 minutes and then $4 \mathrm{~mL} \mathrm{H}_{2} \mathrm{O}$ was removed. $2 \mathrm{~mL}$ of hydrated formate resin was added to each column. Prepared samples were loaded into Dowex AG 1-X8 ion-exchange formate columns. The columns were washed with $5 \mathrm{~mL}$ of $60 \mathrm{mM}$ ammonium formate followed by elution with $2.5 \mathrm{~mL}$ of $0.8 \mathrm{M}$ ammonium formate/0.1 $\mathrm{M}$ formic acid. Levels of incorporated $\left[{ }^{3} \mathrm{H}\right]$-inositol in IP3 were measured by a scintillation counter (Beckman Coulter, Inc., Fullerton, CA).

\section{G. DNA Transfection}

Transfections of DNA were performed with DOTAP liposomal transfection reagent (Roche) according to manufacturer's instructions. Dominant negative Grm1 constructs were provided by Dr. Francesconi [47, 48]. DNA transfections were done with $0.5 \mu \mathrm{g}$ of DNA per $60 \mathrm{~mm}$ plate. 


\section{H. MTT Cell Proliferation Assays}

MTT assays were performed according to manufacturer's protocol (Roche). Briefly, $10^{3}$ cells were plated in quadruplicates in 96-well plate and treated with various compounds as indicated. Absorbance was measured by GENios plate reader (Tecan, Durham, NC) for the time points indicated.

\section{Measurement of Extracellular Glutamate}

Amplex Red Glutamic Acid/Glutamate Oxidase assay kit (Invitrogen-Molecular Probe) was used to measure the amount of glutamate released in the media. Cells were grown in media devoid of glutamate and glutamine, but supplemented with GlutaMax ( $2 \mathrm{mM})$ for 3 days. Cells were plated at $10^{3}$ cells/well with $200 \mu \mathrm{L}$ of media containing specific compounds with concentration as indicated in 96-well plate. After a specified time, 100 $\mu \mathrm{L}$ of media was collected for measurement of the amount of glutamate released, according to manufacturer's protocol. To $50 \mu \mathrm{L}$ of samples, stock or diluted, or standards, $50 \mu \mathrm{L}$ of master mix of Amplex red, peroxidase, glutamate oxidase, glutamate pyruvate transaminase, and alanine was added. During a 30 minutes incubation in a $37^{\circ} \mathrm{C}$ incubator protected from light, glutamate is oxidized by glutamate oxidase to $\alpha-$ ketoglutarate, ammonia, hydrogen peroxide. Alanine and $\alpha-$ ketoglutarate are converted to more glutamate by glutamate pyruvate transaminase. Hydrogen peroxide, produced in these reactions, reacts with Amplex red reagent by peroxidase to form a fluorescent product. Hydrogen peroxide is used as a positive control and glutamate is used as a standard. Fluorescence was measured by a fluorescent microplate reader using excitation at $550 \mathrm{~nm}$ and emission detection at $590 \mathrm{~nm}$. Cells left with approximately $100 \mu \mathrm{L}$ of 
media in the wells were used to confirm the viability of cells by MTT cell proliferation assays.

\section{J. Cell Cycle Analysis}

Cells were plated at $2 \times 10^{6}$ per $100 \mathrm{~mm}$ culture plate and treated as indicated. After 24 and 48 hours, cells were collected and washed twice with ice-cold PBS. Cell pellets were fixed by dropwise addition of ice-cold $70 \%$ ethanol and incubated for 20 minutes at $4^{\circ} \mathrm{C}$. Fixed cells were washed twice with ice-cold PBS and resuspended in $500 \mu \mathrm{L}$ of PBS. Cells were treated with RNaseA solution $(20 \mu \mathrm{g} / \mathrm{mL})$ (Sigma), and labeled with propidium iodide $(50 \mu \mathrm{g} / \mathrm{mL})$ (Sigma) for 30 minutes. Cell cycle analysis was performed by the Flow Cytometry Facility Core at Rutgers University using a Beckman Coulter system (EPICS XL-MCL model).

\section{K. Xenografts in Immunodeficient Nude Mice}

All animal studies were approved by the Institutional Review Board for the Animal Care and Facilities Committee of Rutgers University. Nude mice were purchased from Taconic (Hudson, NY). Human melanoma cells, C8161, were injected into dorsal sites at $10^{6}$ cells per site. Tumor size was measured twice a week with a vernier caliper and calculated as described [93]. Treatment with either vehicle (DMSO) or $7.5 \mathrm{mg} / \mathrm{kg}$ Riluzole was administered daily via oral gavage or intravenously (IV), when tumor volumes reached 6 $\mathrm{mm}^{3}$. After 18 days of treatment, experiments were terminated due to tumor burden as tumor volume had reached $300 \mathrm{~mm}^{3}$ in some animals. 


\section{RNA Interference}

The sequence for Grm1 siRNA was designed according to Ambion, Inc. (Austin, TX) and searched by BLAST to confirm the only sequence homology was to Grm1. Synthesized oligonucleotides by Integrated DNA Technologies (Coralville, IA) were ligated into pSilencer 2.0-U6-hygro expression vectors (Ambion) according to manufacturer's protocol. GFP siRNA oligonucleotides were a negative control purchased from Ambion. Competent E. coli cells were transformed with Grm1 siRNA and GFP siRNA plasmids and siRNA sequences were verified by DNA sequencing (The UMDNJ-RWJMS DNA Sequencing and Synthesis Core Facility, Piscataway, NJ). Transfections of plasmid DNA were performed with DOTAP liposomal transfection reagent (Roche) according to manufacturer's instructions. DNA transfections were done with $2.5 \mu \mathrm{g}$ of DNA per 60 $\mathrm{mm}$ plate. Stable clones were isolated using $125 \mu \mathrm{g} / \mathrm{mL}$ hygromycin in siRNA transfected C8161 cells.

\section{BRAF Kinase Assay}

BRAF kinase cascade assay kit (Upstate, Charlottesville, VA) was used to detect the involvement of BRAF in Grm1 signaling. Assay was carried out according to manufacturer's protocol with some modifications. In short, protein lysates were collected and BRAF was immunoprecipitated using BRAF antibody (Upstate). After immunoprecipitation, the kinase cascade reaction was carried out by adding inactive

unphosphorylated MEK1 and inactive unphosphorylated ERK2 with ${ }^{32} \mathrm{P}-\gamma \mathrm{ATP}$. Active phosphorylated BRAF was used as a positive control. A 10\% SDS-PAGE gel was run and exposed to film overnight. Another type of kinase reaction was carried out only with 
inactive MEK1 without radioactivity. The reaction was terminated using SDS sample buffer. A regular western immunoblot was carried out using phosphor-MEK antibody (Cell signaling technology, Danvers, MA). 


\section{RESULTS}

\section{PART I: HUMAN MELANOMA AND GRM1}

\section{A. GRM1 Expression in Human Melanoma Biopsy Samples}

Following up with the verification that Grm1 is involved in melanoma development in the TG-3 transgenic mouse model, several human melanoma biopsy samples were examined for GRM1 expression. GRM1 expression was demonstrated by four methods: reverse-transcriptase polymerase chain reaction (RT-PCR), quantitative reversetranscriptase polymerase chain reaction (Q-PCR), western immunoblots, and tissue array by immunohistochemistry (IHC). First, RNA was isolated from benign nevi and human melanoma biopsy samples at different stages: primary melanoma, in-transit metastasis, nodal metastasis or recurrent melanoma. After a reverse-transcriptase reaction, duplex PCR was performed to examine GRM1 expression in semi-quantitative manner by including a second set of primers for dopachrome tautomerase (DCT). Only melanoma biopsy samples expressed GRM1, while all samples including benign nevi expressed DCT (Figure 5) [40]. Next, in order to examine GRM1 amount in more quantitative manner, Q-PCR was performed using different sets of RNA isolated from human melanoma biopsy samples, congenital nevi, and human brain. Data were normalized by the expression of tubulin according to a validation worksheet using comparative threshold cycle $\left(\mathrm{C}_{\mathrm{T}}\right)$ analysis (Personal Communication, Dr. Andrew Brooks, Bionomics Research and Technology Center, Environmental and Occupational Health Science Institute, Piscataway, NJ). Congenital nevi are shown as a negative control, which did not 
pass the threshold after 40 cycles suggesting no GRM1 expression (Figure 6). Compared to the human brain sample, GRM1 expression in human melanoma samples varied from $0.04 \%$ (sample 10, passed threshold at 38.6 cycles) to $83 \%$ (sample 4 , passed threshold at 29.5 cycles) of the amount expression in human brain. These RT-PCR data suggest that GRM1 is ectopically expressed in some cases of human melanoma.

In order to verify RT-PCR data, GRM1 protein expression was examined. The same set of human melanoma biopsy tissues from Q-PCR was used for western immunoblots. GRM1 expression was detected in melanoma biopsy samples except in 1 and 5 (Figure 7). Human embryonic kidney cells (HEK293a), normal primary human epidermal melanocytes (HEM) and UACC930 are used as negative controls. HEK293a and HEM are known to be GRM1-negative (unpublished observation) and UACC930 is a human melanoma cell line with a truncation mutation in the coding region of GRM1 (Personal Communication, Dr. Pam Pollock, The Translational Genomics Research Institute, Phoenix, AZ). A transversion ( $\mathrm{C}$ to $\mathrm{T}$ ) at nucleotide 626 resulted in a premature termination at amino acid 131. GRM1 expression is detected in 10 out of 12 human melanoma biopsy samples with varying amounts of GRM1 protein (Samples 2, 3, 4, 6, 7, 8, 9, 10, 11 and 12). Discrepancies between GRM1 RNA expression (Q-PCR) and protein (western) was noted in biopsy samples. By Q-PCR, sample 10 had trace amounts of GRM1 expression (Figure 6), however, by western immunoblots, GRM1 protein was visible (Figure 7). One possible explanation may be the presence of melanin. Some melanomas are highly pigmented and melanin interferes with RT-PCR/Q-PCR; therefore no detectable expression would be appeared. A custom tissue array was made and 
performed by Cybrdi Inc. (Frederick, MD). Representative images are shown (Figure 8). A total of 38 malignant melanoma samples, 15 normal skins and 1 intradermal nevus in duplicates were on the array. Approximately 40\% (15/38) malignant melanomas from various sites expressed GRM1, while none of the normal samples (15 normal skins and 1 nevus) had any detectable GRM1 expression.

\section{B. GRM1 Expression in Human Melanoma Cell Lines}

Human melanoma cell lines were obtained from Dr. Meenhard Herlyn (the Wistar Institute, Philadelphia, PA), Dr. Jeffrey Trent (the Translational Genomics Research Institute, Phoenix, AZ), and Dr. Mary J. C. Hendrix (Children's Memorial Research Center, Chicago, IL). Examination of GRM1 expression was performed at levels of both RNA and protein. RNA was extracted from several human melanoma cell lines as well as normal human melanocytes (NHM102, Dr. Estela E. Medrano, Huffington Center on Aging, Baylor College of Medicine, Houston, TX) and Chinese Hamster Ovary (CHOK1). RT-PCR was first performed with GAPDH primers to examine the integrity of RNA followed by GRM1 primers. The human melanoma cell lines examined had detectable GRM1 expression except UACC930, a GRM1-negative human melanoma cell line. NHM102 and CHOK1 expressed very little or no GRM1 transcripts (Figure 9).

Previously we showed GRM1 expression in several human melanoma cell lines by western immunoblots [40]. An example of immunoblots of several human melanoma cell lines and normal primary human epidermal melanocytes (HEM) is shown (Figure 10). Expression of GRM1 was detected in some human melanoma cell lines, but not in 
UACC930, C81-61, or HEM. We further examined GRM1 expression by immunostaining using antibodies for GRM1 and a melanocyte-specific marker (Tyrp1). The positive control was a normal mouse melanocyte cell line (Melan-A) transfected with Grm1 cDNA. In Figure 11, the left columns represent GRM1 stained cells (red); the middle columns represent Tyrp1 stained cells (green); the right columns represent merged images of two (yellow). GRM1 expression was detected only in human melanoma cell lines and the positive control, while positive Tyrp1 was detected in all melanocytes and melanoma samples (Figure 11). GRM1-positive cell lines were selected for further studies and the list of cell lines used in these studies is shown in Table 1. In most of studies below, HEM is used as a negative control.

\section{Functional GRM1 in Human Melanoma Cells}

A constitutively activated MAPK pathway, specifically extracellular-signal regulated kinase (ERK), has been reported for human melanoma cell lines and biopsies [45, 69, 85]. In these earlier studies, the presence of a BRAF activating mutation (V600E) is the driving force for the activation of ERK. MAPK was shown by many investigators to be an important signaling pathway in melanoma [45, 84]. Our laboratory showed the same pathway to be critical in our system [47]. We demonstrated that stimulation of Grm1 by its agonist, L-quisqualate, leads to the activation of MAPK in TG-3 mouse melanoma derived cell lines [47].

We showed GRM1 expression in several human melanoma cell lines (Figures 9, 10 and 11). Genotypes of BRAF and NRAS were assessed by DNA sequencing in several 
GRM1-positive human melanoma cell lines and those are listed in Table 1. C8161 did not have the most common mutations at either BRAF (codon 600) or NRAS (codons 12, 13 and 61). Most of the other cell lines showed the most common BRAF mutation (V600E), such as UACC903 and WM115. WM239A displayed a different mutation in BRAF (V600D) and a mutation in NRAS (Q61R).

Previously we demonstrated in TG-3 mouse melanoma derived cell lines that Grm1 signals through a Gq protein, followed by phospholipase C (PLC) leading to activation of second messengers, diacylglycerol (DAG) and inositol-1,4,5-triphosphate (IP3) [47]. To examine the functionality of GRM1 in human melanoma cell lines C8161 (Figure 12) and WM239A (Figure 13), the cells were stimulated with GRM1-agonist, L-quisqualate (Q), and the accumulation of IP3 was measured in four groups of cells: no treatment (NT), serum as a positive control (Ser), treatments with Group I mGluR agonist (Q), or preincubation with GRM1 specific antagonist, LY367385, followed by induction with Q (LY/Q). In order to minimize the amount of glutamate, the natural ligand of GRM1, glutamate and glutamine free media were used for the measurement of IP3 supplemented with GlutaMax. We performed a minimum of three independent experiments with each cell line. In the presence of serum, an increase in levels of IP3 accumulation was detected when compared to no treatment (NT). Treatment with GRM1-agonist (Q) for 15 minutes resulted in a statistically significant increase in the accumulation of IP3 for both C8161 (Figure 12) and WM239A (Figure 13). The specificity of Q-induced increase in IP3 accumulation was demonstrated by the absence of IP3 accumulation when these cells were pretreated with GRM1-antagonist (LY367385) followed by induction with Q. 
The functionality of GRM1 in these human melanoma cells was further confirmed by GRM1-agonist (Q) induced ERK phosphorylation (Figure 14). Cells were grown in RPMI1640 without glutamate and glutamine supplemented with GlutaMax and 10\% dialyzed FBS (dFBS) for three days. At 24 hours prior to induction experiments, cells were serum starved. Cells were induced with $\mathrm{Q}$ for up to 10 minutes or pretreated with LY367385 for 30 minutes prior to Q-induction. Western immunoblots were performed to examine the status of ERK phosphorylation. In C8161 or WM239A cells, Q-induced ERK activation was inhibited when these cells were pretreated with LY367385 for 30 minutes prior to induction with Q. However, in UACC903 human melanoma cell line, ERK phosphorylation was not detected after Q induction, while the high basal level of phosphorylated ERK was detected. This observation was likely due to the activating mutation of BRAF (V600E) in UACC903. The V600E BRAF mutation is a strong activating mutation leading to ERK phosphorylation. The GRM1 signal to ERK is likely mediated by RAF (MAPK kinase kinase, MAPKKK); therefore, the activating mutation of BRAF is masking the effects of GRM1 stimulation. As a consequence, stimulation with GRM1-agonist would not be expected to lead to further activation of ERK. This notion was confirmed by a lack of induction by GRM1-agonist in WM115 cells, which also bear the V600E BRAF mutation (data not shown). Another human melanoma cell line UACC930 did not show any induction by GRM1-agonist. This was most likely due to the absence of functional GRM1 receptor. Taken together, we demonstrated that MAPK pathway can be activated in GRM1-positive human melanoma cell lines (C8161 and WM239A) by GRM1-agonist in the absence of the V600E BRAF activating mutation. These results demonstrated that GRM1 receptors in C8161 and WM239A 
human melanoma cell lines were functional and responded to GRM1 agonist and antagonist. 


\section{PART II: SUPPRESSION OF GRM1 SIGNALING}

\section{A. Induction of Apoptosis by Dominant Negative Mutants of GRM1}

To further investigate GRM1 functionality and activity in human melanoma cells, three different but complimentary means were used to suppress GRM1 function. First, we took advantage of the availability of dominant negative mutants of GRM1 [48]. These mutants have a small deletion ( $\triangle \mathrm{CT}$ 694-695, $\Delta \mathrm{CT}$ ) or single base substitutions (P698R, F781S, and F781P) in the intracellular loop 2 or 3, which had been demonstrated to be critical in GRM1 signaling [47, 48]. Human melanoma cell lines, C8161 (Figure 15) and WM239A (Figure 16) were transfected with vector control (Vec) or four different dominant negative GRM1 (dnGRM1) mutants. At 24 hours post-transfection, protein lysates were collected for western immunoblots. The levels of exogenously transfected GRM1 in these cell lines were verified by the use of an anti-Grm1 antibody (Second panels) that only recognizes the rodent forms of Grm1 because these dnGRM1 clones were made from a wild type Grm1 cDNA from rat brain [48]. The same gels were stripped and re-probed with anti-GRM1 antibody that recognizes both human and rodent GRM1 to demonstrate the presence of total GRM1 in these cells (Third panels). Tubulin is used as loading control (Bottom panels). Poly (ADP-ribose) polymerase (PARP) cleavage is a well known apoptotic marker by the appearance of the cleaved form at $89 \mathrm{kDa}$. PARP cleavage was detected only in dnGRM1-transfected samples, but not in vector control (Top panels). Cellular apoptosis is only observed in samples that had been transfected with dominant negative mutants of GRM1. 


\section{B. Inhibition of Human Melanoma Cell Proliferation by GRM1 Antagonists}

Several GRM1 antagonists had been described previously [94]. They are divided into two major groups, competitive and non-competitive. LY367385, a competitive antagonist, binds to the same site as the natural ligand, glutamate [49]. BAY36-7620, one of the noncompetitive antagonists, binds to the transmembrane domain of the receptor resulting in stabilization of the inactive conformation [51]. As a second approach, we used GRM1 antagonists to examine GRM1 functionality and activity in human melanoma cells by the MTT cell proliferation assay.

\section{LY367385, the Competitive Antagonist of GRM1}

Human melanoma cells were grown in the presence of different concentrations of LY367385 in RPMI media devoid of glutamate and glutamine supplemented with GlutaMax to prevent LY367385 from competing with the natural ligand, glutamate, for the binding to GRM1 receptor. The growth of primary human epidermal melanocytes (HEM) requires special media supplemented with several growth factors, which contain approximately $70 \mu \mathrm{M}$ glutamate. In the absence of these factors and glutamate, the growth of HEM was inhibited. Therefore, human embryonic kidney cells (HEK) were used as a normal control instead of HEM. Cell proliferation was measured for four days and the growth of C8161 was inhibited by $70 \%$ in the presence of $500 \mu \mathrm{M}$ LY367385 (Figure 17). There was only a negligible effect on the growth of HEK cells at this concentration. In a parallel set of cells under the same growth conditions at Day 4, levels of phospho-ERK were measured. A dose-dependent decrease in levels of phospho-ERK was detected in cells treated with $100 \mu \mathrm{M}$ or $500 \mu \mathrm{M}$ LY367385, in comparison to no 
treatment (NT) or vehicle treated (Veh) (Figure 18). Quantitation of levels of pERK/ERK relative to NT is shown in the bottom panel (Figure 18). These results demonstrated that treatment of GRM1-positive human melanoma cells with LY367385, a competitive GRM1 antagonist, resulted in suppression in cell proliferation and that this suppression is likely, in part, due to inhibition of MAPK signaling as indicated by a decrease in levels of activated phospho-ERK (Figure 17 and 18). MTT assay on cell proliferation of WM239A cells is shown in Figure 19. While $500 \mu \mathrm{M}$ LY367385 suppressed C8161 cell growth by $70 \%$, there was only a $12 \%$ inhibition of growth in WM239A under such condition. This outcome might be due to the fact that WM239A carries Q61R NRAS mutation and V600D BRAF mutation, prevailing over the effect of GRM1-competitive antagonist or inducing effects that might interfere with the work of LY367385. Two human melanoma cell lines bearing V600E BRAF mutation were also examined. $500 \mu \mathrm{M}$ LY367385 had little or no growth inhibitory effect on the growth of UACC903 (Figure 20) or WM115 (Figure 21). Since BRAF is a downstream target of GRM1-activated MAPK signaling leading to cell proliferation, activating mutation of BRAF (V600E) may be sufficient to maintain constitutive activation of ERK leading to cell proliferation.

\section{BAY 36-7620, the Non-Competitive Antagonist of GRM1}

Next, the growth of human melanoma cells was examined in the presence of a GRM1 non-competitive antagonist, BAY36-7620. As a non-competitive antagonist, BAY367620 does not compete for the binding site with the natural ligand, glutamate; therefore, regular growth media were used for both human melanoma cells and HEM. MTT cell proliferation assays were performed with different concentrations of BAY36-7620 (10 to 
$50 \mu \mathrm{M}$ ) for four days and only data for day 4 is shown (Figure 22). At $30 \mu \mathrm{M}$ BAY367620 , only $30 \%$ of C 8161 cells remain viable, while more than $60 \%$ of HEM cells were viable. Two other human melanoma cell lines were also examined for possible suppression of cell growth. BAY36-7620 was shown to possess $50 \%$ growth inhibitory activity only at concentrations above $50 \mu \mathrm{M}$ in WM239A cells (Figure 23) and

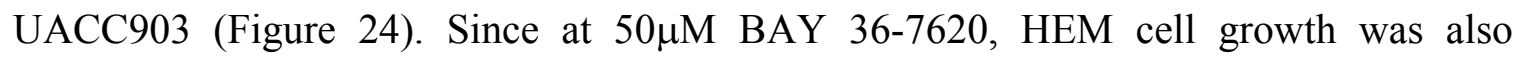
inhibited, the suppression of growth in WM239A and UACC903 by BAY 36-7620 is not likely specific to the suppression of the GRM1 signaling. These MTT assays by GRM1antagonists suggest NRAS and BRAF mutations play a role in the cell proliferation in addition to GRM1.

Cell cycle analysis was done in BAY36-7620-treated C8161 cells (Figure 25). Cell cycle analysis showed close to two-fold increase in the sub-G1 phase from $2.6 \%$ (NT) or 3.3\% (vehicle) to $5.9 \%$ after 24 hours (Table 2) of treatment suggesting an induction of apoptosis by BAY36-7620. After 48 hours (Table 3) of treatment, an induction of apoptosis by BAY36-7620 was further increased from 9.74\% (NT) or 7.62\% (vehicle) to 18.62\%. In BAY36-7620 treated WM239A, cells were accumulated in G2/M phase after 24 hours (Figure 26, Table 6). In UACC903 (Figure 27), BAY36-7620 treatments induced the S phase arrest and increases in the sub-G1 Phase after 24 hours (Table 8) and 48 hours (Table 9). These data suggest GRM1 signaling may play a critical role(s) in human melanoma cells as shown by data from cell cycle analysis induced by GRM1specific antagonist. Apoptosis marker, PARP cleavage was assessed in BAY 36-7620 treated C8161 cells in similar conditions as described for cell cycle analysis. Cleaved 
form of PARP was detected in C8161 cells treated with $50 \mu \mathrm{M}$ of BAY 36-7620 after 48 hours in comparison to controls (Figure 28). Taken together, these data indicated that a non-competitive antagonist of GRM1, BAY36-7620, inhibited human melanoma cell growth and induced apoptosis suggesting that GRM1 could be a target in human melanoma therapy.

\section{Suppression of GRM1 Expression by RNA Interference (RNAi)}

The third way to evaluate the critical role of GRM1 in human melanoma, we used RNAi to inhibit GRM1 function(s) and activities. Normal mouse melanocytes (Melan-A) transfected with Grm1 cDNA (Melan-A-mGluR1-3-1) were used as a positive control. RNAi sequence for Grm1 was designed from the Ambion website (Austin, TX) and cloned into the small interfering RNA (siRNA) expression vector (pSilencer ${ }^{\circledR} 2.0-\mathrm{U} 6$ ) according to the manufacturer's protocol. Stable clones of Grm1 siRNA or GFP siRNA (a negative control) were established in Melan-A-mGluR1-3-1. Western immunoblots were performed to examine the suppression of Grm1 expression; an example is shown in Figure 29. Depending on the clones isolated, there was up to $80 \%$ suppression of Grm1 protein expression in these cells. The same plasmids were used in human cell experiments. Grm1 siRNA or GFP siRNA were transfected into C8161 human melanoma cell line and stable clones were isolated. First, the stable clones were examined for GRM1 expression. Compared to C8161 parent cells, cells transfected with Grm1 siRNA suppressed the GRM1 protein expression (Figure 30). MTT cell proliferation assays were performed to examine the effects of suppression of GRM1 expression in human melanoma cell lines. C8161 cells transfected with Grm1 siRNA had reduced rates of cell 
growth compared to cells transfected with GFP siRNA (Figure 31). Compared to the parent cells, even GFP siRNA transfected cells seem to have slightly reduced cell growth rates, however in Grm1 siRNA clones, the growth rates were lower. Taken together, these results confirmed the importance of GRM1 signaling in human melanoma, especially in cells without mutations in BRAF and NRAS genes. 


\section{PART III: GLUTAMATE SIGNALING IN MELANOMA}

\section{A. Glutamate Release in Melanoma}

In recent years, glutamate signaling in cancer has been a focus of investigation by several laboratories. The antiproliferation potential of iGluR antagonists has been examined in several types of cancers including breast, colon, astrocytoma and lung [92]. Treatment of tumor cells with iGluR antagonists results in inhibition of tumor cell proliferation and motility [92]. Dizocilpine, the iGluR antagonist, can suppress the growth of metastatic lung adenocarcinoma through inhibition of the MAPK signaling pathway [93]. In addition to iGluR and Grm1, metabotropic glutamate receptor 4, has been reported to be overexpressed in colorectal cancer [91]. These studies implicate the involvement of glutamate signaling in tumor development through metabotropic and/or ionotropic glutamate receptors.

It was puzzling to see only $15 \%$ inhibition of melanoma cell growth by $100 \mu \mathrm{M}$ LY367385 in C8161 cells (Figure 17), while $10 \mu \mathrm{M}$ LY367385 was sufficient in suppression of Q-induced ERK activation (Figure 14). Studies on mouse melanoma cells and mouse melanocytic clones stably expressing Grm1 showed higher levels of released glutamate than normal mouse melanocytes or vector controls (S. Shin, manuscript in preparation). In light of these results, levels of released glutamate by several human melanoma cell lines were examined. Each day, half of the media were collected and the amount of released glutamate was determined. MTT assays were performed to ensure that these cells were viable. Again, since HEM, normal human melanocytes, required 
growth factors as well as glutamate to grow, HEK cells were used as a control. All human melanoma cells examined released more glutamate than HEK (Figure 32, Top and Middle Graphs). UACC930, a human melanoma cell line which does not express GRM1, had the lowest amount of glutamate released into the media among human melanoma cell lines examined. In fact, substantial amount of glutamate was released into the media, especially by C8161 cells. After four days, C8161 released close to $200 \mu \mathrm{M}$ glutamate into the media, which was approximately ten times the amount released by HEK. MTT assay on cells demonstrate that cell lines were viable. Although very little glutamate was released by HEK cells, MTT assay demonstrated their vigorous growth (Figure 32, Bottom Graph).

\section{B. Inhibition of Glutamate Release by GRM1 Antagonists in Human Melanoma}

Next, we were interested to know if GRM1-antagonsists could inhibit the release of glutamate by these cells. Treatment of C8161 cells with LY367385 resulted in a dose and time-dependent suppression in the levels of released glutamate (Figure 33). When C8161 cells were treated with $500 \mu \mathrm{M}$ LY367385, less than $30 \%$ of glutamate was released compared to controls, which correlated with MTT assays. Under the same conditions, very little influence on the glutamate released was detected in LY367385 treated HEK cells (Figure 34). These results suggested that a higher concentration of LY367385 was required to inhibit GRM1-positive melanoma cell growth due to the constant release of glutamate by these cells. Suppression of glutamate release was also detected in cells treated with BAY36-7620, the non-competitive GRM1-antagonist (Figure 35). BAY367620 appeared to be more potent in the suppression of glutamate release than LY367385. 


\section{Inhibition of Cell Proliferation by the Glutamate Release Inhibitor, Riluzole}

Riluzole is a Federal Drug Administration (FDA) approved drug for amyotrophic lateral sclerosis (ALS, also known as Lou Gehrig's disease). ALS is a degenerative motor neuron disease that gets progressively worse with time. The actual cause of ALS is not known, however excessive glutamate, a major neurotransmitter, has been proposed to be one of the factors that promotes neuronal excitotoxicity leading to ALS [95]. In clinical trials, the inhibitor of glutamate release, Riluzole, is demonstrated to slow down the progression of ALS [96, 97].

Our previous experiment showed a correlation between levels of released glutamate and cell proliferation (see above). Based on these results, human melanoma cells were treated with Riluzole. Riluzole-treated C8161 cells released reduced levels of glutamate (Figure 36). Suppression of glutamate release by Riluzole also reduced the growth of C8161 human melanoma cells (Figure 37). At $40 \mu \mathrm{M}$ Riluzole, less than $25 \%$ of C8161 cells were viable, while more than $70 \%$ of HEM cells were viable, suggesting that HEM cells were less sensitive under similar conditions. In WM239A (Figure 38) and UACC903 (Figure 39), Riluzole treatment also suppressed melanoma cell proliferation by $50-60 \%$. These data suggest that glutamate release inhibition might be a good therapeutic target.

Cell cycle analysis on Riluzole-treated C8161 cells showed the substantial accumulation of cells in the G2/M phase at 24 hours (Figure 40, Top). By 48 hours, there was a substantial increase in cells accumulated in the sub-G1 phase of the cell cycle suggesting cellular apoptosis (Figure 40, Bottom). The percent of cells in each phase is shown in 
Table 4 (24 hours) and Table 5 (48 hours). Treatment or WM239A cells with Riluzole also showed accumulation of cells in the sub-G1 and G2/M phases of cell cycle (Figure 41, Table 7). In addition, UACC903 also demonstrated that treatments with Riluzole arrested melanoma cells in G2/M phase leading to apoptosis as shown in Figure 42. The percentage of cells in each phase is shown in Table 10 (24 hours) and Table 11 (48 hours). To confirm these observations, PARP cleavage was examined by western immunoblots with C8161 cell lysates prepared at 24 hours and 48 hours post-Riluzole treatment. After 24 and 48 hours treatment with 10,25 and $50 \mu \mathrm{M}$ Riluzole, cleaved forms of PARP were detected in C8161 and WM293A (Figure 43). These results indicated that treatments with Riluzole inhibited growth of human melanoma cells and induced cell cycle arrest leading to apoptosis. These results prompted us to validate the anti-proliferative, pro-apoptotic action of Riluzole in human melanoma cells in vivo.

\section{Inhibition of Human Melanoma Cell Xenograft Growth by Riluzole}

C8161 cells were inoculated subcutaneously into nude mice at $10^{6}$ cells per site. Based on the experiments done previously by others, the maximum tolerated dose of Riluzole [98] was $20 \mathrm{mg} / \mathrm{kg}$. Mice treated with this dose for 2 years had no carcinogenic effects. Based on this information and our pilot experiments, mice were treated with $7.5 \mathrm{mg} / \mathrm{kg}$ Riluzole either by oral gavage or intravenously when tumor volume had reached $6 \mathrm{~mm}^{3}$. Mice were treated every day for 18 days, and tumor sizes were measured twice a week with a vernier caliper. A significant reduction in tumor volume was observed only in mice treated with $7.5 \mathrm{mg} / \mathrm{kg}$ Riluzole by either IV (Figure 44) or oral gavage (Figure 45), compared to untreated (NT) or vehicle treated (Veh) controls. 
In order to examine whether Riluzole treatment also induced apoptosis in melanoma tissue samples, tissue sections were cut and stained for apoptosis. ApopTag In Situ Apoptosis Detection Kit was used to detect apoptotic cells in Riluzole treated samples. In C8161 xenograft models treated with Riluzole, apoptotic cells were detected regardless of mode of administration in these mice (Figure 46). Taken together, these results suggest the possibility of using Riluzole to treat human melanoma. 


\section{PART IV: UACC930, A GRM1-NEGATIVE HUMAN MELANOMA CELL LINE}

UACC930 is a human melanoma cell line that carries a truncation mutation in the coding region of GRM1. In order to examine whether re-introduction of GRM1 has any growth advantage in these cells, Grm1 cDNA was transfected into UACC930. Stable clones were isolated and western immunoblots were performed to examine the GRM1 expression. A representative western is shown in Figure 47. Some stable clones expressed Grm1, while none of the vector controls clones showed Grm1. MTT cell proliferation assay was done on these stable Grm1 clones to examine whether Grm1 expression give these cells a growth advantage (Figure 48). Even though GRM1 plays a critical role in other human melanoma cell lines, re-introduction of Grm1 in UACC930 had little or no effect on growth. Grm1 stable clones were at best growing at the same rate, if not lower, as in the parent cells. Since Grm1 cDNA used was cloned from mouse cDNA library, we could not rule out rodent Grm1 not being optimal in interacting with G proteins or other interacting proteins in the human cells. Currently, cloning of GRM1 cDNA from human origin is ongoing 


\section{PART V: INVOLVEMENT OF BRAF KINASE IN GRM1 SIGNALING IN MOUSE MELANOMA}

MAPK is one of the key signaling pathways in human melanoma [84]. Constitutively activated MAPK pathway, specifically extracellular-signal regulated kinase (ERK), has been reported for human melanoma cell lines and biopsies $[45,69,85]$. In these earlier studies, the presence of BRAF activating mutation (V600E) is the driving force for the activation of ERK. We were interested to know if TG3 derived mouse melanoma cell lines bear BRAF activating mutation similar to human melanoma. RT-PCR followed by sequencing revealed that none of mouse melanoma cell lines in our laboratory bear BRAF activating mutation (unpublished observation).

Previously, our laboratory showed MAPK to be an important signaling pathway in melanoma cell lines derived from our transgenic lines [47]. We also demonstrated that stimulation of Grm1 by its agonist, L-quisqualate (Q), leads to the activation of mitogen activated protein kinase (MAPK) in mouse melanoma cell lines [47]. We hypothesized that cascading signals to MAPK is likely through BRAF; therefore, BRAF kinase assays were performed to examine the involvement of BRAF in Grm1 signaling pathway. First, protein extracts were collected from $\mathrm{Nu} 2$ mouse melanoma cell lines induced with $\mathrm{Q}$ for 5 or 15 minutes or pre-treated with LY367385 for 30 minutes prior to Q induction. BRAF

protein was immunoprecipitated and kinase assays were performed using ${ }^{32} \mathrm{P}-\gamma$-ATP and inactive MEK and inactive ERK. Protein gels were run and exposed to film overnight to show kinase activity of BRAF in these samples (Figure 49). Because the induction 
samples did not show significant differences between them, concerns were raised over high kinase activity in negative control. These high non-specific bands in negative controls were due to inactive ERK, which contained up to $10 \%$ active proteins. To reduce the non-specific bands, similar kinase assays were performed without adding inactive ERK, which contains enough active ERK to mask any real kinase activity. In addition, instead of using radioactivity, western immunoblots were performed for phospho-MEK in these samples (Figure 50). In the western immunoblots, the phospho-MEK level in the negative control was kept low. When $\mathrm{Nu} 2$ mouse melanoma cells were induced with $\mathrm{Q}$ for 5 minutes, the phospho-MEK level was increase in BRAF kinase assay suggesting that BRAF plays a role in the Grm1 signaling cascade. 


\section{DISCUSSION}

\section{A. GRM1 Expression in Human Melanoma}

Several GPCRs have been reported to be involved in carcinogenesis. For example, a GPCR, smoothened (SMO) has been reported to be constitutively activated in $30-40 \%$ of basal cell carcinoma [1]. A tumor suppressor patched homologue 1 (PTCH1) has mutations which inhibits the interactions between PTCH1 and SMO leading to the constitutive activation of SMO. Constitutively activated SMO leads to cancer cell proliferation. Another GPCR, endothelin receptors, has been implicated in melanoma cell proliferation. Overexpression of endothelin receptor B (ETRB) has been detected in human melanoma [99]. An antagonist to ETRB, BQ788, reduces viable cells in vitro by inducing apoptosis in these cells $[42,100]$. Further examination by xenograft models suggested that intratumor injections of BQ788 suppressed melanoma growth in vivo [42]. In addition to previously mentioned GPCRs, we identified metabotropic glutamate receptor 1 (Grm1) to be involved in melanoma development [40]. These growing numbers of evidence suggest the involvement of GPCRs in regulation of cell proliferation and GPCRs act as oncogenes in some cases; therefore, G-protein coupled receptors are now a new class of therapeutic targets in cancer.

Grm1 is normally expressed and functional in the central nervous system. Grm1 is associated with excitatory responses through strong presynaptic stimulation. Grm1 modulates synaptic transmission at the post-synaptic end and modulate neurotransmitter release from pre-synaptic terminals [101]. Unlike voltage-gated ion channels, which are 
involved in the fast response, metabotropic receptors are involved in the slow response by regulating gene expressions through G-proteins. In studies done in our laboratory on TG3 mouse melanoma cell lines, ectopically expressing Grm1 signals through PKCe to the MAPK pathway [47]. MAPK, in turn, could activate several transcription factors including Elk-1 and CREB, leading to cell proliferation. Investigation into human melanoma biopsy samples and cell lines suggested that GRM1 might also play a role in melanoma development in human. Approximately $40 \%$ of human melanoma expressed GRM1, signifying the importance of this GPCR in human melanoma.

Unlike in mouse models, however, the deregulation of GRM1 expression in human melanoma is not known. One possibility is the promoter is in the de-methylated state, an epigenetic alteration that is associated with regulation of gene expressions. For example, RAS association domain family protein-1 (RASSF1) is a RAS binding protein that is regulated by methylation [102]. RASSF1 expression is reduced in $22 \%$ of melanoma biopsy samples and 50\% of melanoma cell lines [102]. When cells are demethylation by treatments with 5-aza-2'-deoxycytidine and trichostatin A, RASSF1 is re-expressed [102]. It would have been more interesting to demonstrate whether re-expressed RASSF1 or general demethylation actually suppressed tumor growth in vivo; nevertheless, RASSF1 is a key example of a gene that whose expression is regulated by methylation. In addition, histone methylation of chromatin surrounding GRM1 in mature human cerebellar cortex suggests the regulation of GRM1 expression by methylation [103]. High levels of dimehtyl-H3-lysine 4 (H3K4me2) is associated with open chromatin [103]. More transcripts of GRM1 are correlated with high levels of H3K4me2 at the GRM1 
promoter region [103], suggesting methylation at the promoter of GRM1 is involved in some cell types. The promoter region of GRM1 is currently being examined for the CpG islands and the possible regulation through methylation in our laboratory.

Another possible explanation for aberrant expression of GRM1 in human melanoma is RE-1 silencing transcription factor (REST, also known as NRSF, neuron-restrictive silencer factor). REST/NRSF functions as a transcriptional repressor of neuronal genes by binding to the neuron-restrictive silencer element (NRSE, also known as RE-1) in non-neuronal cells [104]. NRSE has been detected in the promoter region of GRM1. The binding of REST/NRSF to NRSE suggests the repression of gene expression in nonneuronal cells such as in melanocytes; however, the repression by REST/NRSF is not simple due to the complex nature of co-factors and binding affinities involved with the repression [104]. In addition, a dominant negative form of REST (REST4) has been identified [105]; this truncated version of REST has been implicated in the regulation of differential expression of neuronal genes [105]. Involvement of REST/NRSF in cancers has been recently investigated. Glycine receptor belongs to ligand gated chloride channels involved in the inhibitory neurotransmission in central nervous system [106]. In non-neuronal cells, the alpha subunit of glycine receptor is repressed by REST/NRSF; however, in some small cell lung cancer cells, ectopic expression of this subunit is associated with loss of REST/NRSF [106]. Additionally, deletions in the REST locus of chromosome 4 have been detected in $34 \%$ of colon cancer biopsies and cell lines [107], suggesting deregulation of neuronal gene expression might be involved in colon cancer. 
The involvement of REST/NRSF in aberrant GRM1 expression in human melanoma is currently being investigated.

\section{B. GRM1 as a Target for Melanoma Therapy}

In this thesis, we demonstrated the importance of GRM1 signaling in melanoma cell proliferation by suppressing GRM1 signaling pathway in three different ways: dnGRM1, GRM1 antagonists and Grm1 siRNA. Stable clones of human melanoma cells transfected with Grm1 siRNA reduced melanoma cell proliferation rates when compared to parent control or GFP siRNA stable clones. Treatment of human melanoma cells with LY367385, a GRM1 specific competitive antagonist, exerted dose-dependent inhibition of cell proliferation by suppressing the activation of ERK. These results imply that activated GRM1 in human melanoma cells mediates its signals in part through the MAPK cascade, one of the major cell proliferation pathways. In addition, a non-competitive GRM1 antagonist, BAY36-7620, inhibited cell growth and promoted apoptosis. Furthermore, transient transfection of dnGRM1 into human melanoma cells induced apoptosis as shown by PARP cleavage. These results suggest GRM1 is a potential therapeutic target in human melanoma.

Unlike most other available human melanoma cell lines, C8161 carries no known mutation in NRAS or BRAF genes; however, C8161 expresses GRM1. It is interesting that $\mathrm{C} 8161$ releases the most amounts of glutamate possibly to activate the receptor for growth advantage. WM239A carries a Q61R NRAS mutation and V600D BRAF mutation. Unlike UACC903, which carries V600E BRAF mutation and has constitutive 
activated ERK in the absence of glutamate, WM239A does not have constitutively activated ERK in the absence of glutamate. In addition, ERK activation could be stimulated by inducing GRM1 with its agonist in WM239A, while ERK could not be further stimulated by the GRM1 agonist in UACC903. These results suggest that V600E BRAF activating mutation has the high kinase activity leading to ERK activation; Q61R NRAS mutation is likely does not signal directly into ERK activation; and V600D does not behave like V600E mutation. The involvement of BRAF in Grm1 signaling pathway has been investigated using a TG3 derived mouse melanoma cell line. BRAF kinase assay suggests that Grm1 signal is mediate through BRAF; however, previous induction experiments suggested CRAF might also mediate Grm1 signals (unpublished observation). It is possible that $\mathrm{BRAF}$ and $\mathrm{CRAF}$ are compensating each other for the redundancy in the signaling. Participation of particular RAF isoforms as well as the contribution of NRAS in GRM1 signaling is currently being investigated using RNAi in human melanoma cell lines mentioned.

Two GRM1 antagonists utilized in these studies are very different in their characteristics. LY367385 is a GRM1 specific competitive antagonist; BAY 36-7620 is a GRM1 specific non-competitive antagonist. In order to demonstrate the activities of LY367385, cells could not be grown in the presence of glutamate and glutamine, since LY367385 would compete with glutamate for the binding to the receptor. In case of C8161, cells released more than enough glutamate to activate GRM1 after 24 hours; therefore, high concentrations of LY367385 were required to compensate for suppression of melanoma cell growth. When C8161 melanoma cells were treated with BAY 36-7620, unlike in 
UACC903 or WM239A which only $30 \%$ or $50 \%$ cell growth were inhibited, C8161 cell growth was inhibited by $80 \%$. This implies that without NRAS or BRAF mutations, inhibition of GRM1 signaling is enough to inhibit melanoma cell proliferation. In contrast, in the presence of NRAS or BRAF mutation, suppression of GRM1 signaling is only partial to inhibit melanoma cell proliferation. These suggest that the GRM1 signals may diverge to more than one signaling pathway. If GRM1 antagonists are used for melanoma therapy, it might require additional therapeutic agents in combination with GRM1 antagonists to treat melanoma due to partial suppression of melanoma cell proliferation in the presence of BRAF or NRAS mutation.

\section{UACC930}

UACC930 is a human melanoma cell line with a truncation mutation in the coding region of GRM1, therefore UACC930 does not express GRM1. Additionally, UACC930 grew slower than other melanoma cells. For example, doubling time for UACC930 is approximately 37 hours, while doubling times for UACC903 and C8161 are around 24 hours. Re-introduction of Grm1 in UACC930 was a logical choice to investigate the critical role of GRM1 in melanoma cell proliferation. Even though GRM1 plays a critical role in other human melanoma cell lines, re-introduction of the rodent form of Grm1 in UACC930 had little or no effect on growth, suggesting UACC930 has acquired several genetic alterations to become cancer cells. For example, it is interesting to see UACC930 released the least amount glutamate among all melanoma cell lines examined. This suggests that UACC930 does not require glutamate for cell proliferation; therefore, introduction of Grm1 in UACC930 did not have any effect in cell growth. Another 
possible explanation for the negative results is that the Grm1 introduced to the cells were originated from mouse brain cDNA library. Due to differences in the species, G-protein may not interact properly with Grm1; therefore, GRM1 cDNA from human is currently being cloned for the future use in our laboratory. Once new stable clones are isolated, it would be interesting to examine whether there is an increase in the glutamate being released.

\section{Glutamate Signaling as a target for Melanoma Therapy}

The release of glutamate by several human melanoma cell lines has been demonstrated. Treatment of these melanoma cells with GRM1-antagonists or an inhibitor of glutamate release, Riluzole, suppresses glutamate release and cell growth (Figure 51). In addition, Riluzole diminishes tumor growth in a xenograft model of human melanoma cells. Taken together, these results suggest GRM1 and glutamate signaling may be used as novel targets for melanoma therapy.

Glutamate signaling involves a large group of proteins such as metabotropic glutamate receptors, ionotropic glutamate receptors and glutamate transporters with their effectors. Involvement of glutamate signaling in the brain tumor, glioma, has been under investigation for many years $[108,109]$. Recently, the association of glutamate receptors with cancer of non-neuronal origin has been the topic of research by several groups. Several antagonists of iGluR were shown to inhibit tumor growth and decrease in tumor cell motility in cancers including breast, colon, astrocytoma and lung [92]. Dizocilpine, 
iGluR antagonist, has also been shown to suppress the growth of metastatic lung adenocarcinoma through the inhibition of the MAPK signaling pathway [93].

We demonstrated that human melanoma cells released elevated levels of glutamate, the natural ligand of GRM1, into the media. Suppression of release of glutamate by pharmacological agents (LY367385, BAY36-7620 and Riluzole) correlated with a reduction in human melanoma cell growth. The direct involvement of GRM1 in glutamate release has not yet been investigated extensively. However, it has been reported that two systems work together to regulate extracellular glutamate levels: glutamate uptake transporters and cystine-glutamate exchangers [110]. In studies of the rat prefrontal cortex, suppression of glutamate uptake as well as stimulation of Group I mGluRs (Grm1 and Grm5) by their agonist, DHPG $(R, S)$-3,5-dihydroxy-phenylglycine, leads to an increase in the accumulation of extracellular levels of glutamate [110]. This accumulation is inhibited by LY367385, a GRM1-antagonist. The investigators speculated that LY367385 is also an effective antagonist of cystine-glutamate exchangers, however the possibility of GRM1 mediated signaling to cystine-glutamate exchangers could not be ruled out. Together these results suggest that GRM1 or its signaling may be associated with glutamate release.

Glutamate is one of the major excitatory neurotransmitter. There are several regulatory mechanisms involved in regulation of release of glutamate, considering excessive glutamate leads to neuronal cell death, excitotoxicity. Even as low as 2 to $5 \mu \mathrm{M}$ extracellular glutamate is sufficient to cause excitotoxicity in the mammalian central 
nervous system [95]. Regulation of glutamate release in neurons involves ionotropic glutamate receptors, glutamate transporters, as well as G-protein signaling. In glioma, the skull restricts tumor growth due to spatial restriction [109]. It has been hypothesized that in order for glioma cells to overcome the spatial restriction, excessive glutamate is released to kill nearby neurons, therefore creating enough space for tumor cells to grow. Excitotoxicity of neurons by glioma cells has been demonstrated by co-culture of glioma cells with neurons [109]. In addition, when glioma cell clones were implanted to rat brain to examine tumor growth, the sub-clone that release more glutamate developed more aggressive forms of tumors than the original parental glioma cells [108]. Interestingly, brain is one of the common metastatic sites for melanoma [111]. It is possible when melanoma metastasizes to brain, excess glutamate promotes tumor growth and induces excitotoxicity in a very similar mode of action as described for glioma.

Riluzole is the first FDA approved drug for ALS, however there are only slight benefits to overall survival [95]. The mechanisms of action of Riluzole include inhibition of glutamate release, inactivation of voltage-dependent $\mathrm{Na}^{+}$channels, and interference with G-protein dependent signaling [112]. Riluzole protects neurons by suppression of glutamate release, as well as suppression of ion channels. These neuroprotective properties by Riluzole have been used to target several neurological conditions including basal ganglion disease [112]. We showed that treatment of GRM1-positive human melanoma cells with Riluzole results in the reduced levels of released glutamate, the suppression of human melanoma cell growth, as well as induction of apoptosis. Furthermore, Riluzole is shown to be effective in suppression of tumor growth in a 
xenograft model. Taken together these results suggest the involvement of glutamate signaling in human melanoma, and that Riluzole maybe a good candidate for human melanoma therapy.

Glutamate signaling involves metabotropic glutamate receptors, ionotropic glutamate receptors and transporter. We demonstrated that excess amounts of glutamate were released from human melanoma cells. The consequence, other than the activation of GRM1, is unknown. Excessive glutamate induce neuronal excitotoxicity [95]. Several members of glutamate signaling pathway have been detected in keratinocytes [113]. In normal skin, keratinocytes control the growth of melanocytes keeping the 5:1 ratio of keratinocytes to melanocytes [114]. Since melanocytes normally express GRM5, glutamate signaling or release from keratinocytes might have been a mechanism to control melanocyte proliferation. If keratinocytes release excess glutamate, activated GRM5 could lead to apoptosis similar to neurons. However, when melanocytes were transformed to melanoma cells by ectopically expressing GRM1, released glutamate became a mitogen for melanoma cells. These hypothesis also suggest vastly different signals and consequences between GRM1 and GRM5, even though they belong to the same group and are coupled to Gq proteins. This hypothesis could be supported by our mouse models for melanoma. When Grm5 knock-out mice were crossed with TG-3 mice, new transgenic mice developed melanoma [46]. This implies the importance of GRM1 in cell transformation, while GRM5 does not influence melanocytic transformation. It would be interesting to examine whether glutamate signaling is one way of communicating between melanocytes and keratinocytes. 
Several iGluR antagonists were shown to decrease in tumor cell motility and inhibit xenograft tumor growth in cancers including breast, colon, astrocytoma and lung [92]. In addition to ionotropic glutamate receptors, two metabotropic glutamate receptors are linked to cancers: the overexpression of metabotropic glutamate receptor 4 (GRM4) in colorectal cancer [91] and the aberrant expression of GRM1 in human melanoma. As shown in Figure 51, the suppression of GRM1 in human melanoma leads to the inhibition of cell proliferation; in addition, glutamate release is prevented to suppress further stimulation of the receptor. We identified a glutamate release inhibitor, Riluzole, to be effective in treating melanoma in the C8161 xenograft model. These studies implicate the involvement of glutamate signaling in tumor development and progression through metabotropic glutamate receptors, ionotropic glutamate receptors and/or glutamate transporters. Regulation of glutamate signaling could be a key to finding new therapeutic regimens to treat various types of cancers. 


\section{REFERENCES}

1. Rubin AI, Chen EH, Ratner D: Basal-cell carcinoma. $N$ Engl J Med 2005, 353(21):2262-2269.

2. Alam M, Ratner D: Cutaneous squamous-cell carcinoma. $N$ Engl J Med 2001, 344(13):975-983.

3. American Cancer Society: Cancer Facts and Figures 2007. Atlanta: American Cancer Society; 2007.

4. Bowden GT: Prevention of non-melanoma skin cancer by targeting ultraviolet-Blight signalling. Nat Rev Cancer 2004, 4(1):23-35.

5. Huangfu D, Anderson KV: Signaling from Smo to Ci/Gli: conservation and divergence of Hedgehog pathways from Drosophila to vertebrates. Development 2006, 133(1):3-14.

6. MacKie RM: Malignant melanoma: clinical variants and prognostic indicators. Clin Exp Dermatol 2000, 25(6):471-475.

7. Liu Z-J, Herlyn M: Molecular biology of cutaneous melanoma. In: Cancer: Principles and Practice of Oncology. Edited by DeVita VTJ, Hellman S, Rosenberg SA. Philadelphia, PA: Lippincott Williams \& Wilkins; 2005: 17451753.

8. Tsao H, Atkins MB, Sober AJ: Management of cutaneous melanoma. $N$ Engl $J$ Med 2004, 351(10):998-1012.

9. Morton DL, Wen DR, Wong JH, Economou JS, Cagle LA, Storm FK, Foshag LJ, Cochran AJ: Technical details of intraoperative lymphatic mapping for early stage melanoma. Arch Surg 1992, 127(4):392-399.

10. Macripo G, Quaglino P, Caliendo V, Ronco AM, Soltani S, Giacone E, Pau S, Fierro MT, Bernengo MG: Sentinel lymph node dissection in stage I/II melanoma patients: surgical management and clinical follow-up study. Melanoma Res 2004, 14(2):S9-12.

11. National Cancer Institute: Melanoma: Clinical Trial, vol. 2005; 2005.

12. Caraglia M, Vitale G, Marra M, Budillon A, Tagliaferri P, Abbruzzese A: Alphainterferon and its effects on signalling pathways within cells. Curr Protein Pept Sci 2004, 5(6):475-485. 
13. Bart RS, Porzio NR, Kopf AW, Vilcek JT, Cheng EH, Farcet Y: Inhibition of growth of B16 murine malignant melanoma by exogenous interferon. Cancer Res 1980, 40(3):614-619.

14. Romerio F, Zella D: MEK and ERK inhibitors enhance the anti-proliferative effect of interferon-alpha2b. Faseb J 2002, 16(12):1680-1682.

15. Chawla-Sarkar M, Leaman DW, Borden EC: Preferential induction of apoptosis by interferon (IFN)-beta compared with IFN-alpha2: correlation with TRAIL/Apo2L induction in melanoma cell lines. Clin Cancer Res 2001, 7(6):1821-1831.

16. Slaton JW, Perrotte P, Inoue K, Dinney CP, Fidler IJ: Interferon-alpha-mediated down-regulation of angiogenesis-related genes and therapy of bladder cancer are dependent on optimization of biological dose and schedule. Clin Cancer Res 1999, 5(10):2726-2734.

17. Kefford RF: Adjuvant therapy of cutaneous melanoma: the interferon debate. Ann Oncol 2003, 14(3):358-365.

18. Kirkwood JM, Strawderman MH, Ernstoff MS, Smith TJ, Borden EC, Blum RH: Interferon alfa-2b adjuvant therapy of high-risk resected cutaneous melanoma: the Eastern Cooperative Oncology Group Trial EST 1684. J Clin Oncol 1996, 14(1):7-17.

19. Kirkwood JM, Ibrahim JG, Sondak VK, Richards J, Flaherty LE, Ernstoff MS, Smith TJ, Rao U, Steele M, Blum RH: High- and low-dose interferon alfa-2b in high-risk melanoma: first analysis of intergroup trial E1690/S9111/C9190. J Clin Oncol 2000, 18(12):2444-2458.

20. Kirkwood JM, Manola J, Ibrahim J, Sondak V, Ernstoff MS, Rao U: A pooled analysis of eastern cooperative oncology group and intergroup trials of adjuvant high-dose interferon for melanoma. Clin Cancer Res 2004, 10(5):1670-1677.

21. Lens MB, Dawes M: Interferon alfa therapy for malignant melanoma: a systematic review of randomized controlled trials. J Clin Oncol 2002, 20(7):18181825 .

22. Eggermont AM: The role interferon-alpha in malignant melanoma remains to be defined. Eur J Cancer 2001, 37(17):2147-2153.

23. Eklund JW, Kuzel TM: A review of recent findings involving interleukin-2-based cancer therapy. Curr Opin Oncol 2004, 16(6):542-546.

24. Atkins MB, Sparano J, Fisher RI, Weiss GR, Margolin KA, Fink KI, Rubinstein L, Louie A, Mier JW, Gucalp R et al: Randomized phase II trial of high-dose interleukin-2 either alone or in combination with interferon alfa- $2 \mathrm{~b}$ in advanced renal cell carcinoma. J Clin Oncol 1993, 11(4):661-670. 
25. Radny P, Caroli UM, Bauer J, Paul T, Schlegel C, Eigentler TK, Weide B, Schwarz M, Garbe C: Phase II trial of intralesional therapy with interleukin-2 in soft-tissue melanoma metastases. Br J Cancer 2003, 89(9):1620-1626.

26. Hauschild A, Weichenthal M, Balda BR, Becker JC, Wolff HH, Tilgen W, Schulte KW, Ring J, Schadendorf D, Lischner S et al: Prospective randomized trial of interferon alfa- $2 \mathrm{~b}$ and interleukin- 2 as adjuvant treatment for resected intermediate- and high-risk primary melanoma without clinically detectable node metastasis. J Clin Oncol 2003, 21(15):2883-2888.

27. Atkins MB, Lotze MT, Dutcher JP, Fisher RI, Weiss G, Margolin K, Abrams J, Sznol M, Parkinson D, Hawkins M et al: High-dose recombinant interleukin 2 therapy for patients with metastatic melanoma: analysis of 270 patients treated between 1985 and 1993. J Clin Oncol 1999, 17(7):2105-2116.

28. Thompson JF, Scolyer RA, Kefford RF: Cutaneous melanoma. Lancet 2005, 365(9460):687-701.

29. Dudley ME, Wunderlich JR, Yang JC, Sherry RM, Topalian SL, Restifo NP, Royal RE, Kammula U, White DE, Mavroukakis SA et al: Adoptive cell transfer therapy following non-myeloablative but lymphodepleting chemotherapy for the treatment of patients with refractory metastatic melanoma. J Clin Oncol 2005, 23(10):2346-2357.

30. Eggermont AM, Kirkwood JM: Re-evaluating the role of dacarbazine in metastatic melanoma: what have we learned in 30 years? Eur J Cancer 2004, 40(12):1825-1836.

31. Chapman PB, Einhorn LH, Meyers ML, Saxman S, Destro AN, Panageas KS, Begg CB, Agarwala SS, Schuchter LM, Ernstoff MS et al: Phase III multicenter randomized trial of the Dartmouth regimen versus dacarbazine in patients with metastatic melanoma. J Clin Oncol 1999, 17(9):2745-2751.

32. Greco O, Scott SD, Marples B, Dachs GU: Cancer gene therapy: 'delivery, delivery, delivery'. Front Biosci 2002, 7:d1516-1524.

33. Kaiser J: Gene therapy. Panel urges limits on X-SCID trials. Science 2005, 307(5715):1544-1545.

34. Marshall E: Gene therapy death prompts review of adenovirus vector. Science 1999, 286(5448):2244-2245.

35. Zhang L, Nolan E, Kreitschitz S, Rabussay DP: Enhanced delivery of naked DNA to the skin by non-invasive in vivo electroporation. Biochim Biophys Acta 2002, 1572(1):1-9. 
36. Zhu H, Reuhl K, Botha R, Ryan K, Wei J, Chen S: Development of early melanocytic lesions in transgenic mice predisposed to melanoma. Pigm Cell Res 2000, 13:158-164.

37. Zhu H, Reuhl K, Zhang X, Botha R, Ryan K, Wei J, Chen S: Development of heritable melanoma in transgenic mice. J Invest Dermatol 1998, 110:247-252.

38. Chen S, Zhu H, Wetzel WJ, Philbert MA: Spontaneous melanocytosis in transgenic mice. J Invest Dermatol 1996, 106:1145-1150.

39. Chen S, Tiecher L, Kazim D, Pollack R, Wise L: Commitment of mouse fibroblasts to adipocyte differentiation by DNA transfection. Science 1989, 244:582-585.

40. Pollock PM, Cohen-Solal K, Sood R, Namkoong J, Martino JJ, Koganti A, Zhu H, Robbins C, Makalowska I, Shin SS et al: Melanoma mouse model implicates metabotropic glutamate signaling in melanocytic neoplasia. Nat Genet 2003, 34(1):108-112.

41. Marin YE, Chen S: Involvement of metabotropic glutamate receptor 1, a G protein coupled receptor, in melanoma development. J Mol Med 2004, 82(11):735-749.

42. Lahav R, Heffner G, Patterson PH: An endothelin receptor B antagonist inhibits growth and induces cell death in human melanoma cells in vitro and in vivo. Proc Natl Acad Sci U S A 1999, 96(20):11496-11500.

43. Ferraguti F, Baldani-Guerra B, Corsi M, Nakanishi S, Corti C: Activation of the extracellular signal-regulated kinase 2 by metabotropic glutamate receptors. Eur $J$ Neurosci 1999, 11(6):2073-2082.

44. Thandi S, Blank JL, Challiss RA: Group-I metabotropic glutamate receptors, mGlu1a and mGlu5a, couple to extracellular signal-regulated kinase (ERK) activation via distinct, but overlapping, signalling pathways. J Neurochem 2002, 83(5):1139-1153.

45. Satyamoorthy K, Li G, Gerrero MR, Brose MS, Volpe P, Weber BL, Van Belle P, Elder DE, Herlyn M: Constitutive mitogen-activated protein kinase activation in melanoma is mediated by both BRAF mutations and autocrine growth factor stimulation. Cancer Res 2003, 63(4):756-759.

46. Marin YE, Namkoong J, Shin SS, Raines J, Degenhardt K, White E, Chen S: Grm5 expression is not required for the oncogenic role of Grm1 in melanocytes. Neuropharmacology 2005, 49 Suppl:70-79.

47. Marin YE, Namkoong J, Cohen-Solal K, Shin SS, Martino JJ, Oka M, Chen S: Stimulation of oncogenic metabotropic glutamate receptor 1 in melanoma cells activates ERK1/2 via PKCepsilon. Cell Signal 2006, 18(8):1279-1286. 
48. Francesconi A, Duvoisin RM: Role of the second and third intracellular loops of metabotropic glutamate receptors in mediating dual signal transduction activation. J Biol Chem 1998, 273(10):5615-5624.

49. Clark BP, Baker, S. Richard, Goldsworthy, John, Harris, John R., Kingston, Ann E.: (+)-2-Methyl-4-Carboxylphenylglycine (LY367385) Selectively Antagonises Metabotropic Glutamate mGLuR1 Receptors. Bioorganic \& Medicinal Chemistry Letters 1997, 7(21):2777-2780.

50. Gasparini F, Floersheim P, Flor PJ, Heinrich M, Inderbitzin W, Ott D, Pagano A, Stierlin C, Stoehr N, Vranesic I et al: Discovery and characterization of noncompetitive antagonists of group I metabotropic glutamate receptors. Farmaco 2001, 56(1-2):95-99.

51. Carroll FY, Stolle A, Beart PM, Voerste A, Brabet I, Mauler F, Joly C, Antonicek $\mathrm{H}$, Bockaert J, Muller T et al: BAY36-7620: a potent non-competitive mGlu1 receptor antagonist with inverse agonist activity. Mol Pharmacol 2001, 59(5):965-973.

52. Prezeau L, Gomeza J, Ahern S, Mary S, Galvez T, Bockaert J, Pin JP: Changes in the carboxyl-terminal domain of metabotropic glutamate receptor 1 by alternative splicing generate receptors with differing agonist-independent activity. Mol Pharmacol 1996, 49(3):422-429.

53. Ango F, Pin JP, Tu JC, Xiao B, Worley PF, Bockaert J, Fagni L: Dendritic and axonal targeting of type 5 metabotropic glutamate receptor is regulated by homer1 proteins and neuronal excitation. J Neurosci 2000, 20(23):8710-8716.

54. Ango F, Prezeau L, Muller T, Tu JC, Xiao B, Worley PF, Pin JP, Bockaert J, Fagni L: Agonist-independent activation of metabotropic glutamate receptors by the intracellular protein Homer. Nature 2001, 411(6840):962-965.

55. Smalley KS, Eisen TG: Farnesyl transferase inhibitor SCH66336 is cytostatic, pro-apoptotic and enhances chemosensitivity to cisplatin in melanoma cells. Int $J$ Cancer 2003, 105(2):165-175.

56. Adjei AA: Blocking oncogenic Ras signaling for cancer therapy. J Natl Cancer Inst 2001, 93(14):1062-1074.

57. Marshall CJ: Specificity of receptor tyrosine kinase signaling: transient versus sustained extracellular signal-regulated kinase activation. Cell 1995, 80(2):179185.

58. van Elsas A, Zerp SF, van der Flier S, Kruse KM, Aarnoudse C, Hayward NK, Ruiter DJ, Schrier PI: Relevance of ultraviolet-induced N-ras oncogene point mutations in development of primary human cutaneous melanoma. Am J Pathol 1996, 149(3):883-893. 
59. Eastman A: Activation of programmed cell death by anticancer agents: cisplatin as a model system. Cancer Cells 1990, 2(8-9):275-280.

60. Halaschek-Wiener J, Kloog Y, Wacheck V, Jansen B: Farnesyl thiosalicylic acid chemosensitizes human melanoma in vivo. J Invest Dermatol 2003, 120(1):109115 .

61. Smalley KS, Eisen TG: Farnesyl thiosalicylic acid inhibits the growth of melanoma cells through a combination of cytostatic and pro-apoptotic effects. Int J Cancer 2002, 98(4):514-522.

62. Eskandarpour M, Kiaii S, Zhu C, Castro J, Sakko AJ, Hansson J: Suppression of oncogenic NRAS by RNA interference induces apoptosis of human melanoma cells. Int J Cancer 2005.

63. Storm SM, Cleveland JL, Rapp UR: Expression of raf family proto-oncogenes in normal mouse tissues. Oncogene 1990, 5(3):345-351.

64. Schaap D, van der Wal J, Howe LR, Marshall CJ, van Blitterswijk WJ: A dominant-negative mutant of raf blocks mitogen-activated protein kinase activation by growth factors and oncogenic p21ras. J Biol Chem 1993, 268(27):20232-20236.

65. Wellbrock C, Karasarides M, Marais R: The RAF proteins take centre stage. Nat Rev Mol Cell Biol 2004, 5(11):875-885.

66. Karasarides M, Chiloeches A, Hayward R, Niculescu-Duvaz D, Scanlon I, Friedlos F, Ogilvie L, Hedley D, Martin J, Marshall CJ et al: B-RAF is a therapeutic target in melanoma. Oncogene 2004, 23(37):6292-6298.

67. Wellbrock C, Ogilvie L, Hedley D, Karasarides M, Martin J, Niculescu-Duvaz D, Springer CJ, Marais R: V599EB-RAF is an oncogene in melanocytes. Cancer Res 2004, 64(7):2338-2342.

68. Dumaz N, Hayward R, Martin J, Ogilvie L, Hedley D, Curtin JA, Bastian BC, Springer C, Marais R: In Melanoma, RAS Mutations Are Accompanied by Switching Signaling from BRAF to CRAF and Disrupted Cyclic AMP Signaling. Cancer Res 2006, 66(19):9483-9491.

69. Davies H, Bignell GR, Cox C, Stephens P, Edkins S, Clegg S, Teague J, Woffendin H, Garnett MJ, Bottomley W et al: Mutations of the BRAF gene in human cancer. Nature 2002, 417(6892):949-954.

70. Pollock PM, Harper UL, Hansen KS, Yudt LM, Stark M, Robbins CM, Moses TY, Hostetter G, Wagner U, Kakareka J et al: High frequency of BRAF mutations in nevi. Nat Genet 2003, 33(1):19-20. 
71. Dong J, Phelps RG, Qiao R, Yao S, Benard O, Ronai Z, Aaronson SA: BRAF oncogenic mutations correlate with progression rather than initiation of human melanoma. Cancer Res 2003, 63(14):3883-3885.

72. Goydos JS, Mann B, Kim HJ, Gabriel EM, Alsina J, Germino FJ, Shih W, Gorski DH: Detection of B-RAF and N-RAS mutations in human melanoma. J Am Coll Surg 2005, 200(3):362-370.

73. Halaban R, Ghosh S, Duray P, Kirkwood JM, Lerner AB: Human melanocytes cultured from nevi and melanomas. J Invest Dermatol 1986, 87(1):95-101.

74. Hingorani SR, Jacobetz MA, Robertson GP, Herlyn M, Tuveson DA: Suppression of BRAF(V599E) in human melanoma abrogates transformation. Cancer Res 2003, 63(17):5198-5202.

75. Bhatt KV, Spofford LS, Aram G, McMullen M, Pumiglia K, Aplin AE: Adhesion control of cyclin D1 and p27Kip1 levels is deregulated in melanoma cells through BRAF-MEK-ERK signaling. Oncogene 2005, 24(21):3459-3471.

76. Sumimoto H, Miyagishi M, Miyoshi H, Yamagata S, Shimizu A, Taira K, Kawakami Y: Inhibition of growth and invasive ability of melanoma by inactivation of mutated BRAF with lentivirus-mediated RNA interference. Oncogene 2004, 23(36):6031-6039.

77. Danson S, Lorigan P: Improving outcomes in advanced malignant melanoma: update on systemic therapy. Drugs 2005, 65(6):733-743.

78. Eisen T, Ahmad T, Flaherty KT, Gore M, Kaye S, Marais R, Gibbens I, Hackett $\mathrm{S}$, James M, Schuchter LM et al: Sorafenib in advanced melanoma: a Phase II randomised discontinuation trial analysis. Br J Cancer 2006, 95(5):581-586.

79. Kane RC, Farrell AT, Saber H, Tang S, Williams G, Jee JM, Liang C, Booth B, Chidambaram N, Morse D et al: Sorafenib for the treatment of advanced renal cell carcinoma. Clin Cancer Res 2006, 12(24):7271-7278.

80. Stebbins CE, Russo AA, Schneider C, Rosen N, Hartl FU, Pavletich NP: Crystal structure of an Hsp90-geldanamycin complex: targeting of a protein chaperone by an antitumor agent. Cell 1997, 89(2):239-250.

81. Workman P: Overview: translating Hsp90 biology into Hsp90 drugs. Curr Cancer Drug Targets 2003, 3(5):297-300.

82. Ikenoue T, Hikiba Y, Kanai F, Tanaka Y, Imamura J, Imamura T, Ohta M, Ijichi $\mathrm{H}$, Tateishi K, Kawakami $\mathrm{T}$ et al: Functional analysis of mutations within the kinase activation segment of B-Raf in human colorectal tumors. Cancer Res 2003, 63(23):8132-8137. 
83. Burger AM, Fiebig HH, Stinson SF, Sausville EA: 17-(Allylamino)-17demethoxygeldanamycin activity in human melanoma models. Anticancer Drugs 2004, 15(4):377-387.

84. Ge X, Fu YM, Meadows GG: U0126, a mitogen-activated protein kinase kinase inhibitor, inhibits the invasion of human A375 melanoma cells. Cancer Lett 2002, 179(2):133-140.

85. Huntington JT, Shields JM, Der CJ, Wyatt CA, Benbow U, Slingluff CL, Jr., Brinckerhoff CE: Overexpression of collagenase 1 (MMP-1) is mediated by the ERK pathway in invasive melanoma cells: role of BRAF mutation and fibroblast growth factor signaling. J Biol Chem 2004, 279(32):33168-33176.

86. Favata MF, Horiuchi KY, Manos EJ, Daulerio AJ, Stradley DA, Feeser WS, Van Dyk DE, Pitts WJ, Earl RA, Hobbs F et al: Identification of a novel inhibitor of mitogen-activated protein kinase kinase. J Biol Chem 1998, 273(29):1862318632.

87. Dudley DT, Pang L, Decker SJ, Bridges AJ, Saltiel AR: A synthetic inhibitor of the mitogen-activated protein kinase cascade. Proc Natl Acad Sci U S A 1995, 92(17):7686-7689.

88. Mandic A, Viktorsson K, Heiden T, Hansson J, Shoshan MC: The MEK1 inhibitor PD98059 sensitizes C8161 melanoma cells to cisplatin-induced apoptosis. Melanoma Res 2001, 11(1):11-19.

89. Goodall J, Wellbrock C, Dexter TJ, Roberts K, Marais R, Goding CR: The Brn-2 transcription factor links activated BRAF to melanoma proliferation. Mol Cell Biol 2004, 24(7):2923-2931.

90. Jean D, Tellez C, Huang S, Davis DW, Bruns CJ, McConkey DJ, Hinrichs SH, Bar-Eli M: Inhibition of tumor growth and metastasis of human melanoma by intracellular anti-ATF-1 single chain Fv fragment. Oncogene 2000, 19(22):27212730 .

91. Chang HJ, Yoo BC, Lim SB, Jeong SY, Kim WH, Park JG: Metabotropic glutamate receptor 4 expression in colorectal carcinoma and its prognostic significance. Clin Cancer Res 2005, 11(9):3288-3295.

92. Rzeski W, Turski L, Ikonomidou C: Glutamate antagonists limit tumor growth. Proc Natl Acad Sci USA 2001, 98(11):6372-6377.

93. Stepulak A, Sifringer M, Rzeski W, Endesfelder S, Gratopp A, Pohl EE, Bittigau P, Felderhoff-Mueser U, Kaindl AM, Buhrer C et al: NMDA antagonist inhibits the extracellular signal-regulated kinase pathway and suppresses cancer growth. Proc Natl Acad Sci U S A 2005, 102(43):15605-15610. 
94. Hermans E, Challiss RA: Structural, signalling and regulatory properties of the group I metabotropic glutamate receptors: prototypic family $\mathrm{C} \mathrm{G-protein-coupled}$ receptors. Biochem J 2001, 359(Pt 3):465-484.

95. Van Den Bosch L, Van Damme P, Bogaert E, Robberecht W: The role of excitotoxicity in the pathogenesis of amyotrophic lateral sclerosis. Biochim Biophys Acta 2006.

96. Bensimon G, Lacomblez L, Meininger V: A controlled trial of riluzole in amyotrophic lateral sclerosis. ALS/Riluzole Study Group. N Engl J Med 1994, 330(9):585-591.

97. Bryson HM, Fulton B, Benfield P: Riluzole. A review of its pharmacodynamic and pharmacokinetic properties and therapeutic potential in amyotrophic lateral sclerosis. Drugs 1996, 52(4):549-563.

98. Prescribing Information [http://products.sanofi-aventis.us]

99. Loftus SK, Chen Y, Gooden G, Ryan JF, Birznieks G, Hilliard M, Baxevanis AD, Bittner M, Meltzer P, Trent J et al: Informatic selection of a neural crestmelanocyte cDNA set for microarray analysis. Proc Natl Acad Sci U S A 1999, 96(16):9277-9280.

100. Lahav R, Suva ML, Rimoldi D, Patterson PH, Stamenkovic I: Endothelin receptor $\mathrm{B}$ inhibition triggers apoptosis and enhances angiogenesis in melanomas. Cancer Res 2004, 64(24):8945-8953.

101. Bordi F, Ugolini A: Group 1 metabotropic glutamate receptors: Implications for brain diseases. Progress in Neurobiology 1999, 59:55-79.

102. Reifenberger J, Knobbe CB, Sterzinger AA, Blaschke B, Schulte KW, Ruzicka T, Reifenberger G: Frequent alterations of Ras signaling pathway genes in sporadic malignant melanomas. Int J Cancer 2004, 109(3):377-384.

103. Stadler F, Kolb G, Rubusch L, Baker SP, Jones EG, Akbarian S: Histone methylation at gene promoters is associated with developmental regulation and region-specific expression of ionotropic and metabotropic glutamate receptors in human brain. J Neurochem 2005, 94(2):324-336.

104. Coulson JM: Transcriptional regulation: cancer, neurons and the REST. Curr Biol 2005, 15(17):R665-668.

105. Spencer EM, Chandler KE, Haddley K, Howard MR, Hughes D, Belyaev ND, Coulson JM, Stewart JP, Buckley NJ, Kipar A et al: Regulation and role of REST and REST4 variants in modulation of gene expression in in vivo and in vitro in epilepsy models. Neurobiol Dis 2006, 24(1):41-52. 
106. Neumann SB, Seitz R, Gorzella A, Heister A, Doeberitz MK, Becker CM: Relaxation of glycine receptor and onconeural gene transcription control in NRSF deficient small cell lung cancer cell lines. Brain Res Mol Brain Res 2004, 120(2):173-181.

107. Westbrook TF, Martin ES, Schlabach MR, Leng Y, Liang AC, Feng B, Zhao JJ, Roberts TM, Mandel G, Hannon GJ et al: A genetic screen for candidate tumor suppressors identifies REST. Cell 2005, 121(6):837-848.

108. Takano T, Lin JH, Arcuino G, Gao Q, Yang J, Nedergaard M: Glutamate release promotes growth of malignant gliomas. Nat Med 2001, 7(9):1010-1015.

109. Ye ZC, Sontheimer H: Glioma cells release excitotoxic concentrations of glutamate. Cancer Res 1999, 59(17):4383-4391.

110. Melendez RI, Vuthiganon J, Kalivas PW: Regulation of extracellular glutamate in the prefrontal cortex: focus on the cystine glutamate exchanger and group I metabotropic glutamate receptors. J Pharmacol Exp Ther 2005, 314(1):139-147.

111. Xie TX, Huang FJ, Aldape KD, Kang SH, Liu M, Gershenwald JE, Xie K, Sawaya R, Huang S: Activation of stat3 in human melanoma promotes brain metastasis. Cancer Res 2006, 66(6):3188-3196.

112. Doble A: The pharmacology and mechanism of action of riluzole. Neurology 1996, 47(6 Suppl 4):S233-241.

113. Genever PG, Maxfield SJ, Kennovin GD, Maltman J, Bowgen CJ, Raxworthy MJ, Skerry TM: Evidence for a novel glutamate-mediated signaling pathway in keratinocytes. J Invest Dermatol 1999, 112(3):337-342.

114. Haass NK, Smalley KS, Li L, Herlyn M: Adhesion, migration and communication in melanocytes and melanoma. Pigment Cell Res 2005, 18(3):150-159. 


\begin{tabular}{|l|c|c|c|c|}
\hline Cell Line & Cell Type & GRM1 & NRAS & BRAF \\
\hline CHOK1 & ovary & - & & \\
\hline HEK293A & kidney & - & & \\
\hline HEM & melanocyte & - & & \\
\hline & & & & \\
\hline UACC930 & melanoma & Truncation & & \\
\hline A2058 & melanoma & + & & \\
\hline C8161 & melanoma & + & & \\
\hline UACC903 & melanoma & + & & V600E \\
\hline WM239A & melanoma & + & Q61R & V600D \\
\hline WM115 & melanoma & + & & V600E \\
\hline C81-61 & melanoma & + & & \\
\hline
\end{tabular}

Table 1

List of Cell Lines

NRAS and BRAF mutations are activating mutations, while GRM1 mutation is nonsense mutation. 


\begin{tabular}{|l|c|c|c|c|}
\hline Conditions & Sub-G1 & G0/G1 & S & G2/M \\
\hline No Treatment & 2.60 & 50.36 & 25.44 & 21.60 \\
\hline Vehicle & 3.30 & 48.22 & 25.42 & 23.06 \\
\hline $\begin{array}{l}\text { BAY 36-7620 } \\
\mathbf{1 0 0} \boldsymbol{\mu M}\end{array}$ & $\mathbf{5 . 9 0}$ & 49.60 & 23.82 & 20.68 \\
\hline
\end{tabular}

Table 2

Cell Cycle Analysis: 24 Hours After BAY 36-7620 Treatment of C8161

\begin{tabular}{|l|c|c|c|c|}
\hline Conditions & Sub-G1 & G0/G1 & S & G2/M \\
\hline No Treatment & 9.74 & 55.44 & 17.60 & 17.22 \\
\hline Vehicle & 7.62 & 57.86 & 17.18 & 17.34 \\
\hline $\begin{array}{l}\text { BAY 36-7620 } \\
\mathbf{1 0 0} \mu \mathrm{M}\end{array}$ & $\mathbf{1 8 . 6 2}$ & 32.64 & $\mathbf{2 8 . 2 8}$ & 20.46 \\
\hline
\end{tabular}

Table 3

Cell Cycle Analysis: 48 Hours After BAY 36-7620 Treatment of C8161 


\begin{tabular}{|l|c|c|c|c|}
\hline Conditions & Sub-G1 & G0/G1 & S & G2/M \\
\hline No Treatment & 2.60 & 50.36 & 25.44 & 21.60 \\
\hline Vehicle & 3.30 & 48.22 & 25.42 & 23.06 \\
\hline Riluzole $\mathbf{1 0 0} \mu \mathbf{M}$ & 2.94 & 5.58 & 16.38 & $\mathbf{7 5 . 1 0}$ \\
\hline
\end{tabular}

Table 4

Cell Cycle Analysis: 24 Hours After Riluzole Treatment of C8161

\begin{tabular}{|l|c|c|c|c|}
\hline Conditions & Sub-G1 & G0/G1 & S & G2/M \\
\hline No Treatment & 9.74 & 55.44 & 17.60 & 17.22 \\
\hline Vehicle & 7.62 & 57.86 & 17.18 & 17.34 \\
\hline Riluzole $\mathbf{1 0 0} \mu \mathbf{M}$ & $\mathbf{2 2 . 7 2}$ & 10.32 & $\mathbf{3 0 . 4 4}$ & $\mathbf{3 6 . 5 2}$ \\
\hline
\end{tabular}

Table 5

Cell Cycle Analysis: 48 Hours After Riluzole Treatment of C8161 


\begin{tabular}{|l|r|r|r|r|}
\hline Conditions & Sub-G1 & G0/G1 & \multicolumn{1}{c|}{ S } & G2/M \\
\hline No Treatment & 1.80 & 39.28 & 30.96 & 27.96 \\
\hline Vehicle & 2.94 & 39.66 & 30.30 & 27.10 \\
\hline $\begin{array}{l}\text { BAY36-7620 } \\
\mathbf{1 0 0} \mu \mathrm{M}\end{array}$ & 2.44 & 38.66 & 24.86 & $\mathbf{3 4 . 0 4}$ \\
\hline
\end{tabular}

Table 6

Cell Cycle Analysis: 24 Hours After BAY36-7620 Treatment of WM239A

\begin{tabular}{|l|r|r|c|c|}
\hline Conditions & Sub-G1 & G0/G1 & \multicolumn{1}{c|}{ S } & G2/M \\
\hline No Treatment & 1.80 & 39.28 & 30.96 & 27.96 \\
\hline Vehicle & 2.94 & 39.66 & 30.30 & 27.10 \\
\hline Riluzole $\mathbf{1 0 0} \mu \mathrm{M}$ & $\mathbf{7 . 9 5}$ & 22.50 & 28.88 & $\mathbf{4 0 . 6 6}$ \\
\hline
\end{tabular}

Table 7

Cell Cycle Analysis: 24 Hours After Riluzole Treatment of WM239A 


\begin{tabular}{|l|c|c|c|c|}
\hline Conditions & Sub-G1 & G1 & S & G2/M \\
\hline No Treatment & 1.06 & 52.22 & 25.04 & 23.40 \\
\hline Vehicle & 1.06 & 54.40 & 23.56 & 23.16 \\
\hline $\begin{array}{l}\text { BAY 36-7620 } \\
\mathbf{1 0 0} \mu \mathrm{M}\end{array}$ & $\mathbf{1 1 . 9 2}$ & 46.96 & $\mathbf{2 7 . 1 2}$ & 16.34 \\
\hline
\end{tabular}

Table 8

Cell Cycle Analysis: 24 Hours After BAY 36-7620 Treatment of UACC903

\begin{tabular}{|l|c|c|c|c|}
\hline Conditions & Sub-G1 & G1 & S & G2/M \\
\hline No Treatment & 0.60 & 71.40 & 16.30 & 13.34 \\
\hline Vehicle & 0.82 & 70.21 & 14.55 & 15.77 \\
\hline $\begin{array}{l}\text { BAY 36-7620 } \\
\mathbf{1 0 0} \mu \mathrm{M}\end{array}$ & $\mathbf{2 6 . 5 0}$ & 40.02 & $\mathbf{2 1 . 3 4}$ & 13.68 \\
\hline
\end{tabular}

Table 9

Cell Cycle Analysis: 48 Hours After BAY 36-7620 Treatment of UACC903 


\begin{tabular}{|l|c|c|c|c|}
\hline Conditions & Sub-G1 & G1 & S & G2/M \\
\hline No Treatment & 1.06 & 52.22 & 25.04 & 23.40 \\
\hline Vehicle & 1.06 & 54.40 & 23.56 & 23.16 \\
\hline Riluzole $100 \mu \mathrm{M}$ & $\mathbf{4 . 2 1}$ & 28.78 & 13.61 & $\mathbf{5 4 . 6 9}$ \\
\hline
\end{tabular}

Table 10

Cell Cycle Analysis: 24 Hours After Riluzole Treatment of UACC903

\begin{tabular}{|l|c|c|c|c|}
\hline Conditions & Sub-G1 & G1 & S & G2/M \\
\hline No Treatment & 0.60 & 71.40 & 16.30 & 13.34 \\
\hline Vehicle & 0.82 & 70.21 & 14.55 & 15.77 \\
\hline Riluzole $\mathbf{1 0 0} \mu \mathbf{M}$ & $\mathbf{1 6 . 5 0}$ & 31.98 & $\mathbf{2 7 . 1 2}$ & $\mathbf{2 6 . 1 0}$ \\
\hline
\end{tabular}

Table 11

Cell Cycle Analysis: 48 Hours After Riluzole Treatment of UACC903 


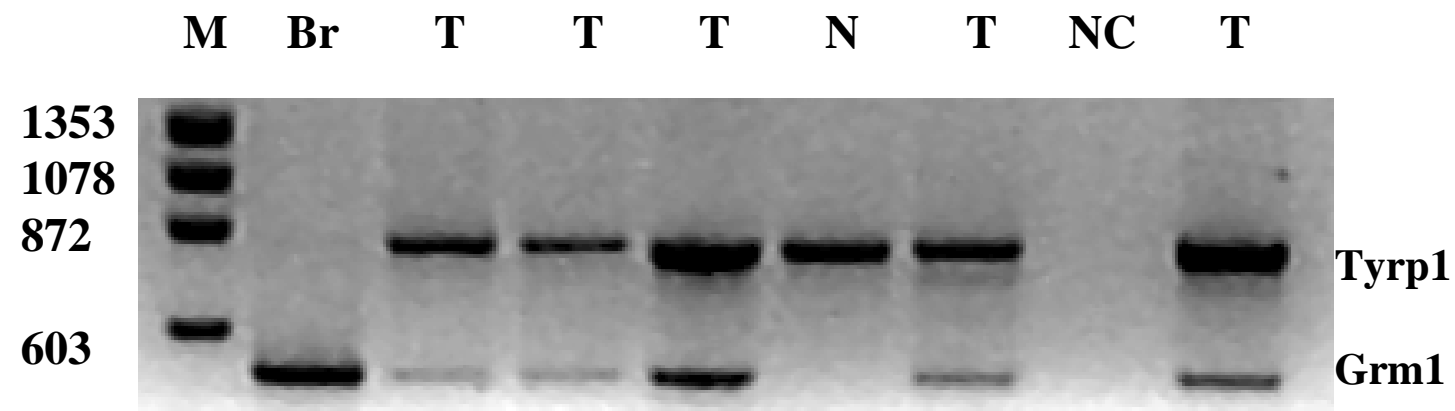

Figure 1

Grm1 Expression in TG-3 Mouse Tumors

Reverse transcriptase-polymerase chain reaction (RT-PCR) was performed from RNA isolated from TG-3 tumor ears, normal C57BL/6 ears and C57BL/6 mouse brain control [41]. The top band represents Tyrp1, melanocyte-specific gene, at 784 bp and the bottom band represents Grm1 at 500 bp. M stands for DNA markers with size (bp) represented on the left side. Br stands for mouse brain control, a positive control for Grm1 expression, T stands for tumor ears isolated from 5 independent TG-3 mice and $\mathrm{N}$ stands for normal ears. NC is a negative control. Expression of Grm1 is detected only in TG-3 tumor ears. The Grm1 bands were normalized to Tyrp1. 


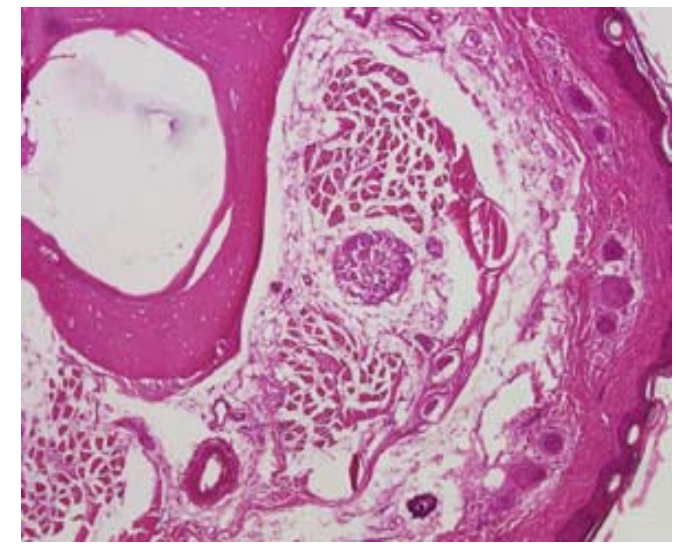

Control Tail

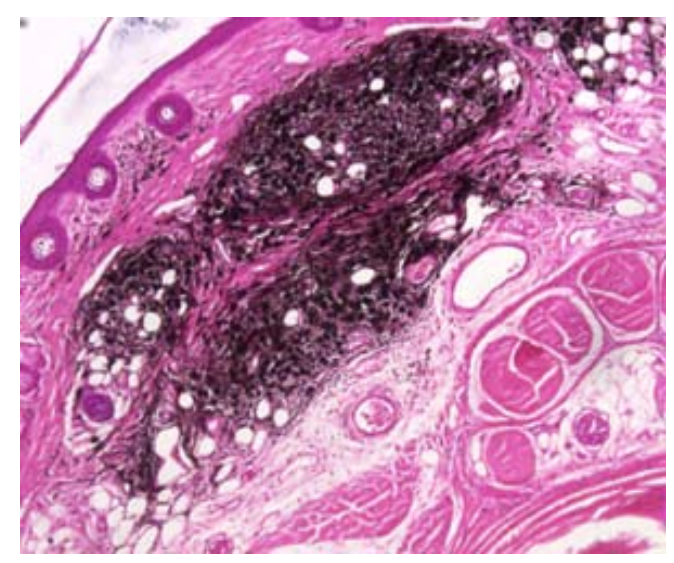

Tumor Tail

Figure 2

Histopathology of Tail Sections of Normal and E-line Mice

Hematoxylin and Eosin (H\&E) staining was performed on normal control tail section and tumor tail section of E-line. Tail section of E-line showed black pigmented lesions, which were melanocytic tumors. 


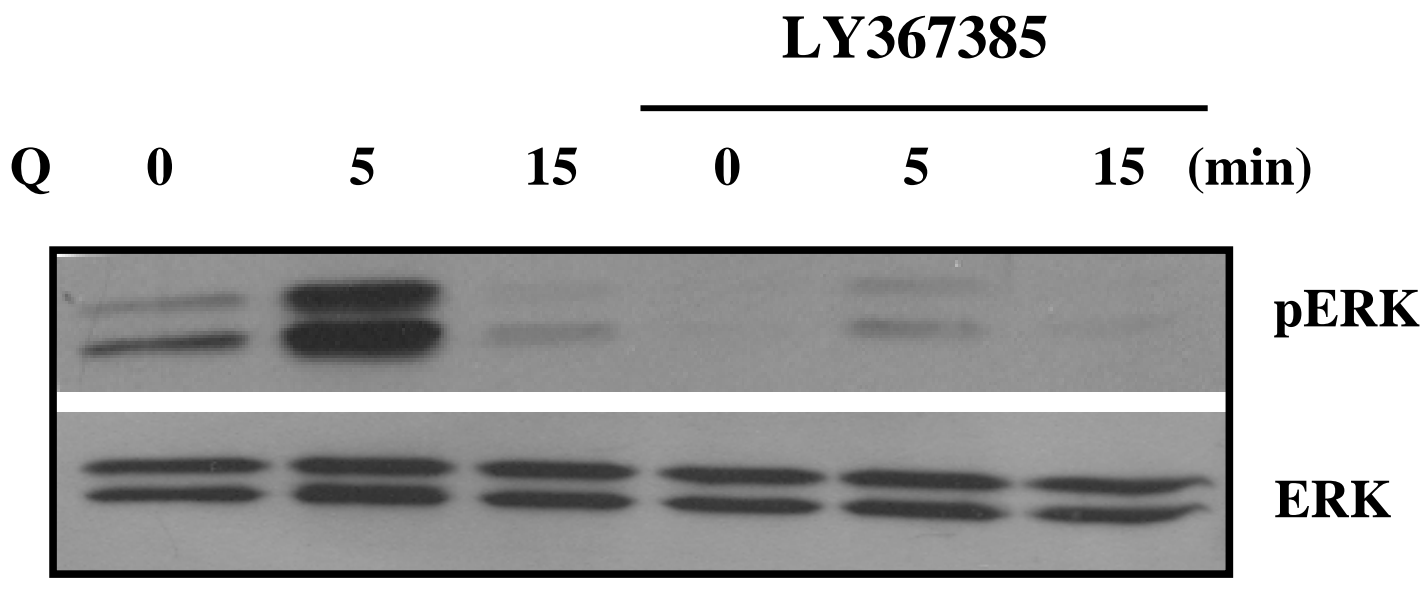

Figure 3

Activation/Inhibition of Grm1 by Agonist (L-quisqualate) and Antagonist (LY367385)

To demonstrate the functionality of Grm1 in Nu2 (TG-3 melanoma cell line), cells were stimulated with L-quisqualate (Q) for 5 and 15 minutes [47]. Preincubation of cells with Grm1-specific antagonist, LY367385, for 30 minutes prior to Q induction, led to suppression of ERK phosphorylation. The same gel was stripped and probed with total ERK for normalization. 


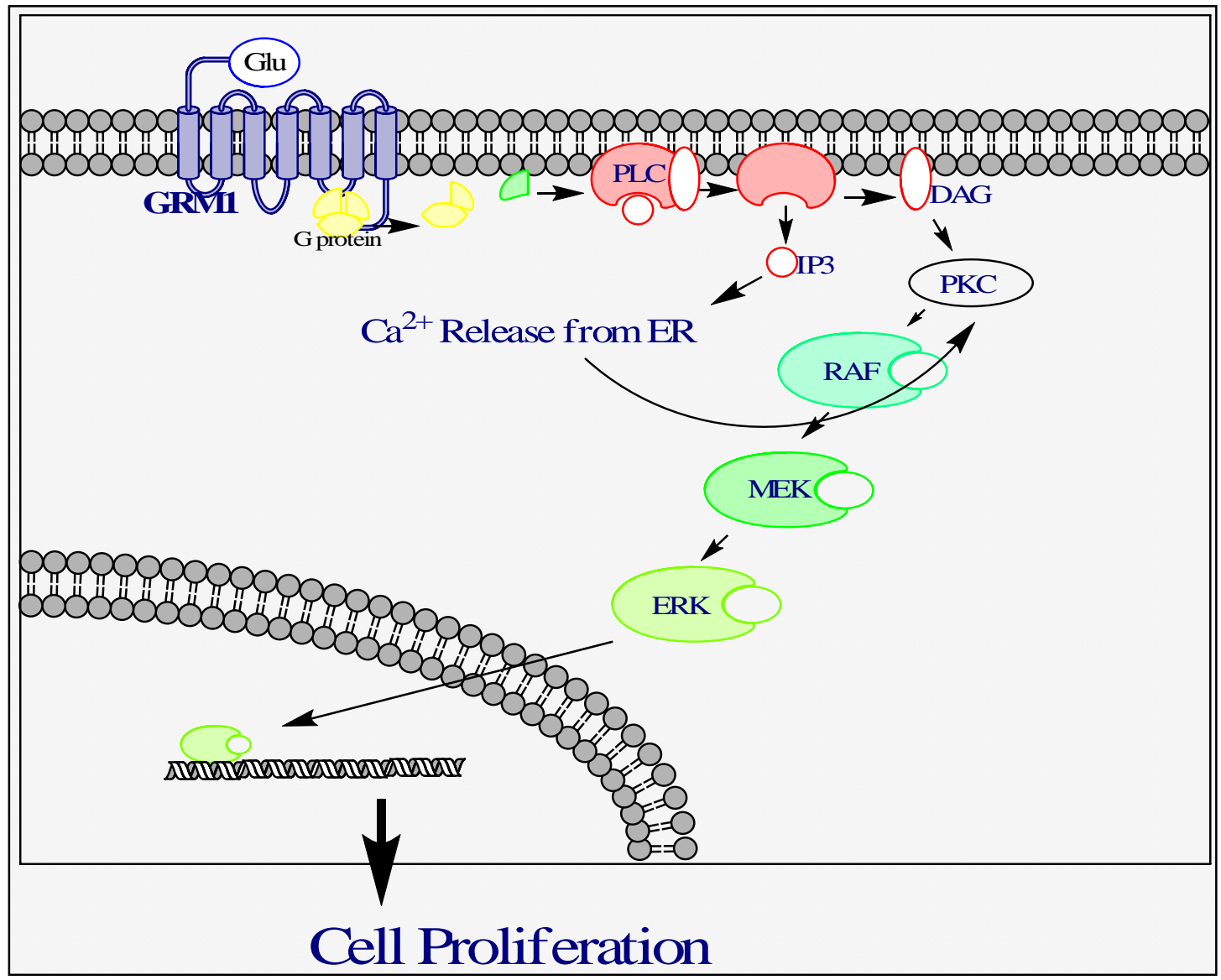

Figure 4

Grm1 Signaling Pathway in Melanoma

From our mouse melanoma cell line signaling studies, we derived the Grm1 signaling pathway. Upon stimulation by its natural ligand, glutamate, Grm1 signals through Gq, leading to the production of diacylglycerol (DAG) and inositol triphosphate (IP3). Accumulations of these second messengers lead to the activation of the RAF/MEK/ERK pathway, mediated through PKC epsilon. ERK translocates to the nucleus activating transcription factors required for melanoma cell proliferation. 


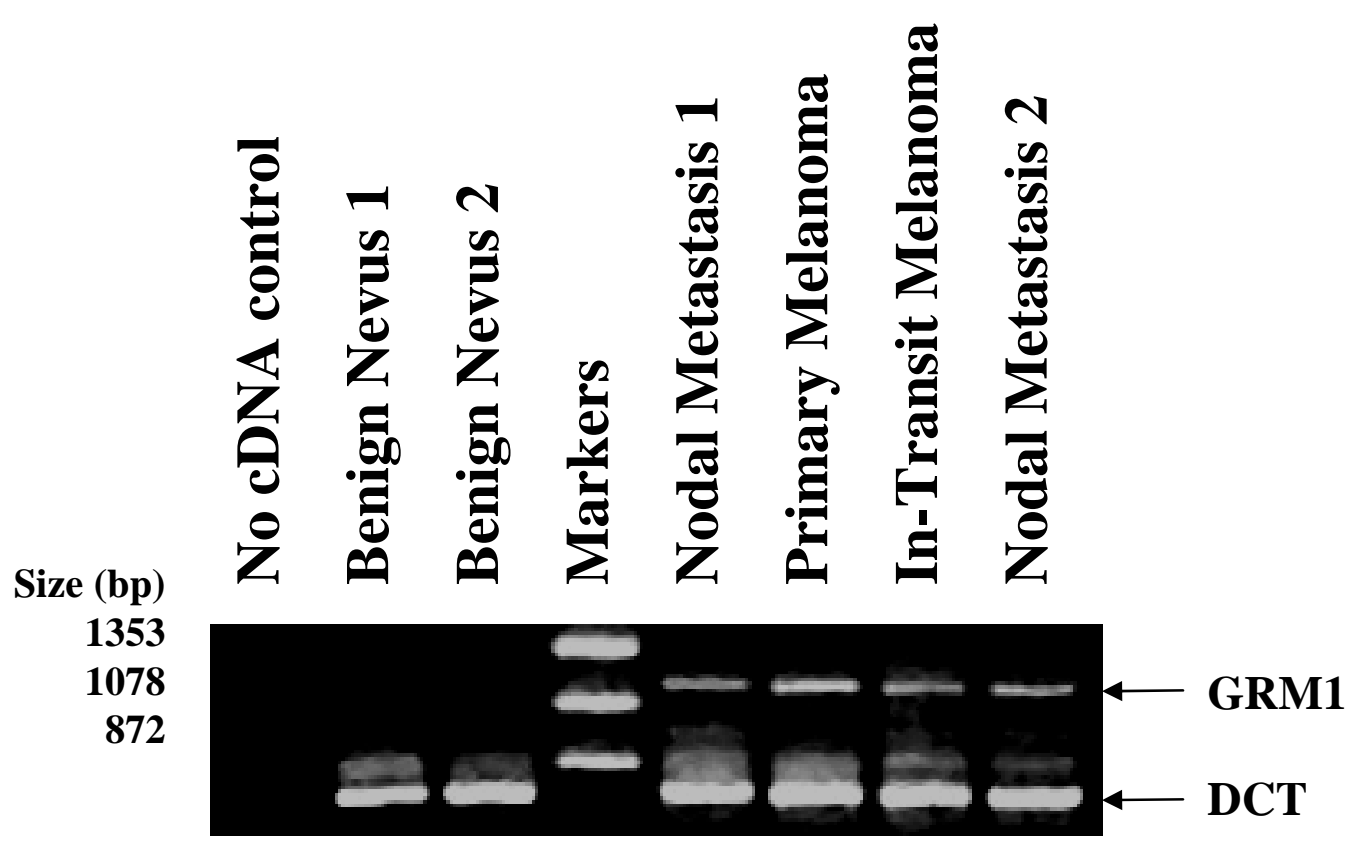

Figure 5

GRM1 Expression in Human Melanoma Biopsy Samples: RT-PCR

RT-PCR was performed from RNA isolated from human melanoma biopsy samples and benign nevi [40]. The top band represents GRM1 at $1.1 \mathrm{~kb}$ and the bottom band represents DCT, dopachrome tautomerase-melanocyte specific gene, at $600 \mathrm{bp}$. Expression of GRM1 was detected only in melanoma biopsies, when compared to DCT that was used for normalization. 


\section{GRM1 Expression}

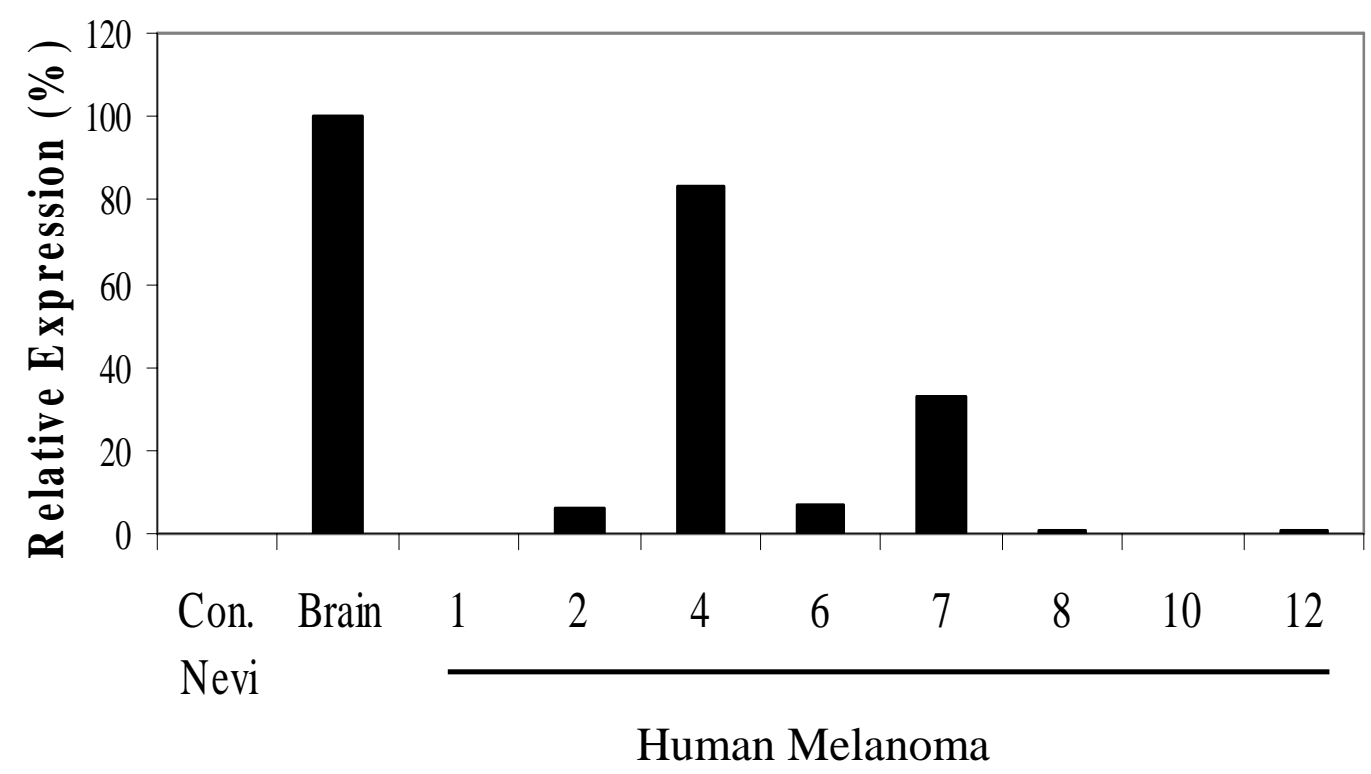

Figure 6

GRM1 Expression in Human Melanoma Biopsy Samples: Q-PCR

Quantitative PCR (Q-PCR) was performed from RNA isolated from human melanoma biopsy samples, congenital nevi, and human brain. Isolated RNA was given to Bionomics Research and Technology Center, a part of core facilities at the Environmental and Occupational Health Science Institute (EOHSI, Piscataway, NJ). Data was normalized to tubulin expression levels. Brain was used as the positive control. GRM1 expression was detected in 4/8 melanoma biopsy samples shown, but not in nevi. 

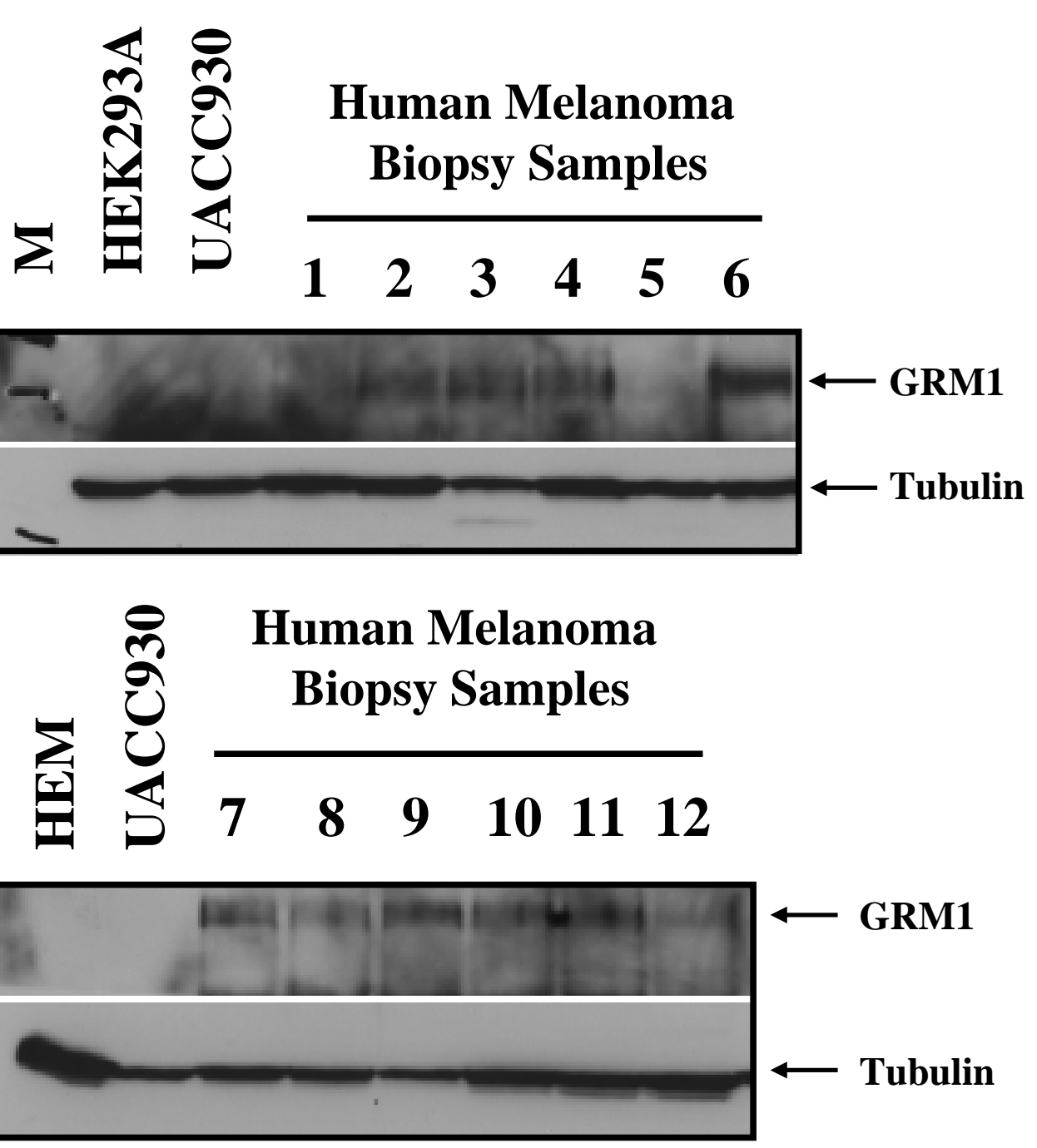

Figure 7

GRM1 Expression in Human Melanoma Biopsy Samples: Western Blots

Protein lysates were extracted from human melanoma biopsy samples. HEK239A are human embryonic kidney cells, known not to express GRM1 protein; UACC930 is a human melanoma cell line with a truncation mutation in GRM1 coding region; and HEM is normal human epidermal melanocyte. GRM1 expression was only apparent in some biopsy samples (samples 2, 3, 4, 6, 7, 8, 9, 10, 11, and 12), but not in HEK293A, UACC930 and HEM. The bottom of the membrane was probed with tubulin. 

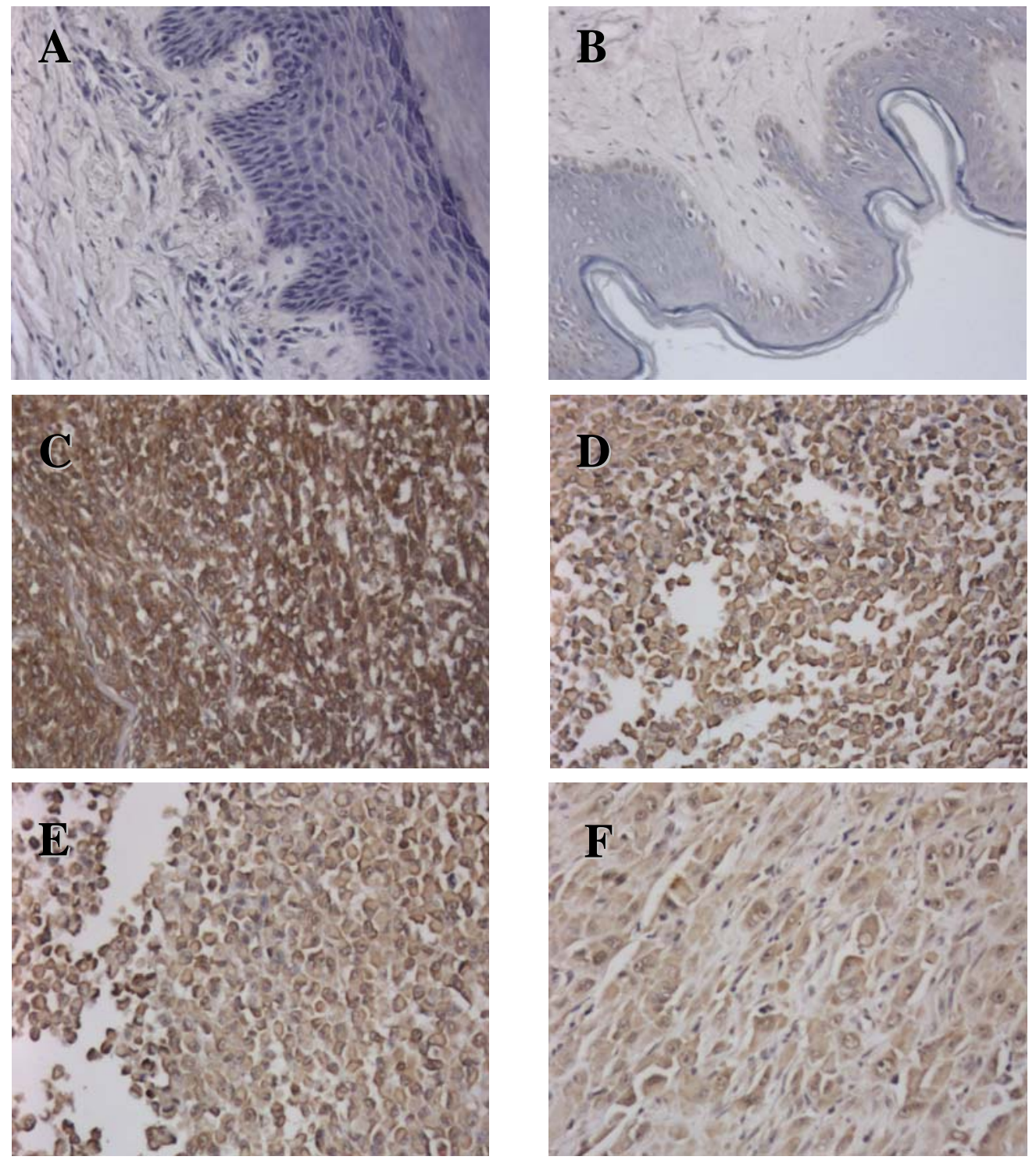

Figure 8

Immunohistochemistry-Human Melanoma Tissue Array

GRM1 expression was examined by immunohistochemistry in the custom tissue array. Human normal skin (A and B) was compared to human melanoma tissues (C-F). When stained with GRM1 antibody, approximately 40\% (15/38) of human melanoma tissues were positively stained whereas none of normal skin samples were stained. 


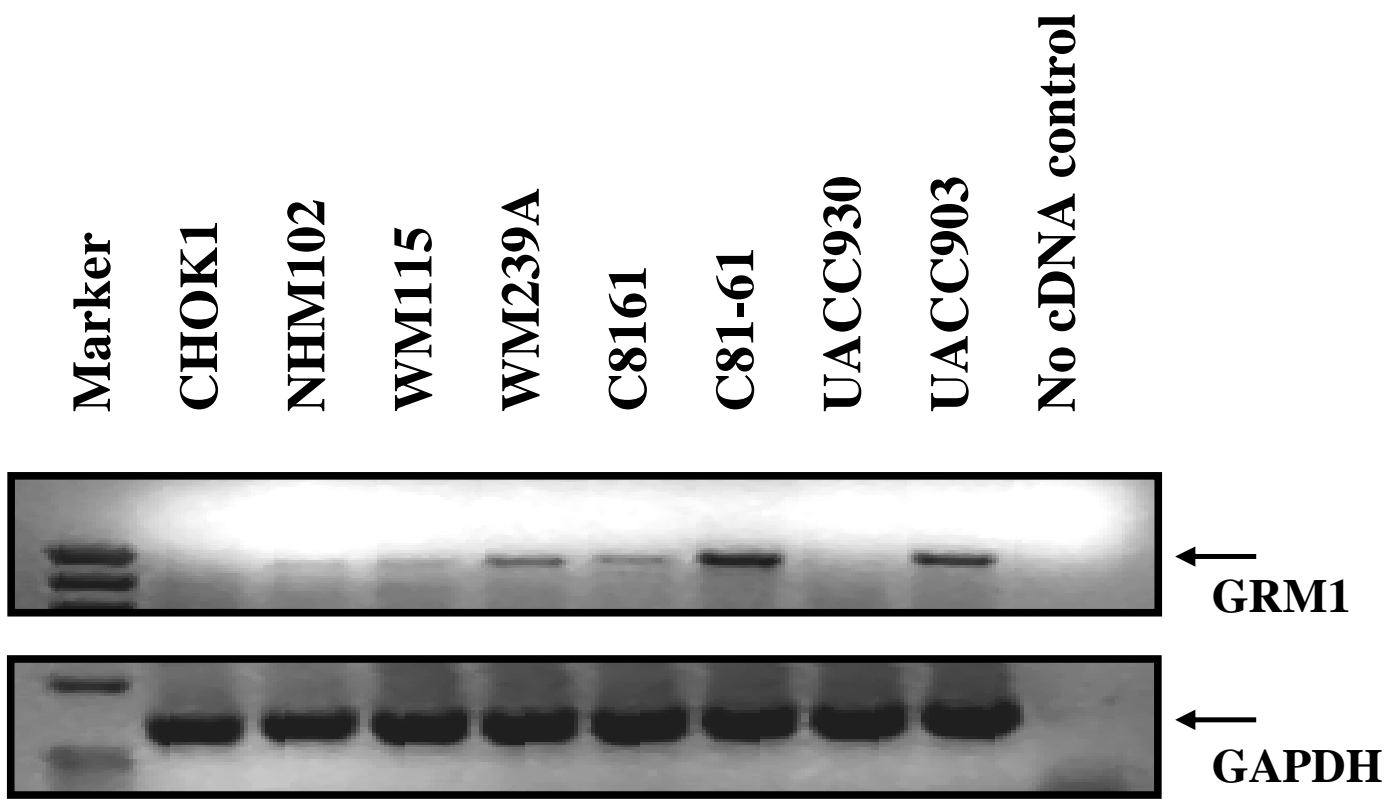

Figure 9

GRM1 Expression in Human Melanoma Cell Lines: RT-PCR

GRM1 expression of human melanoma cell lines was examined by RT-PCR. RNA was extracted from cell lines and RT-PCR was performed with GAPDH primers and GRM1 primers. The top gel showed the expression of GRM1 in these cell lines and the bottom gel showed the GAPDH expression for the integrity of RNA. CHOK1 is Chinese hamster ovary cells; NHM102 is normal human melanocytes; WM115, WM239A, C8161, C81-61, UACC903 and UACC930 are human melanoma cell lines. UACC930 has a truncation mutation in GRM1 coding region, and used as a negative control. Five out of six human melanoma cell lines shown here had detectable GRM1 RNA expression. 


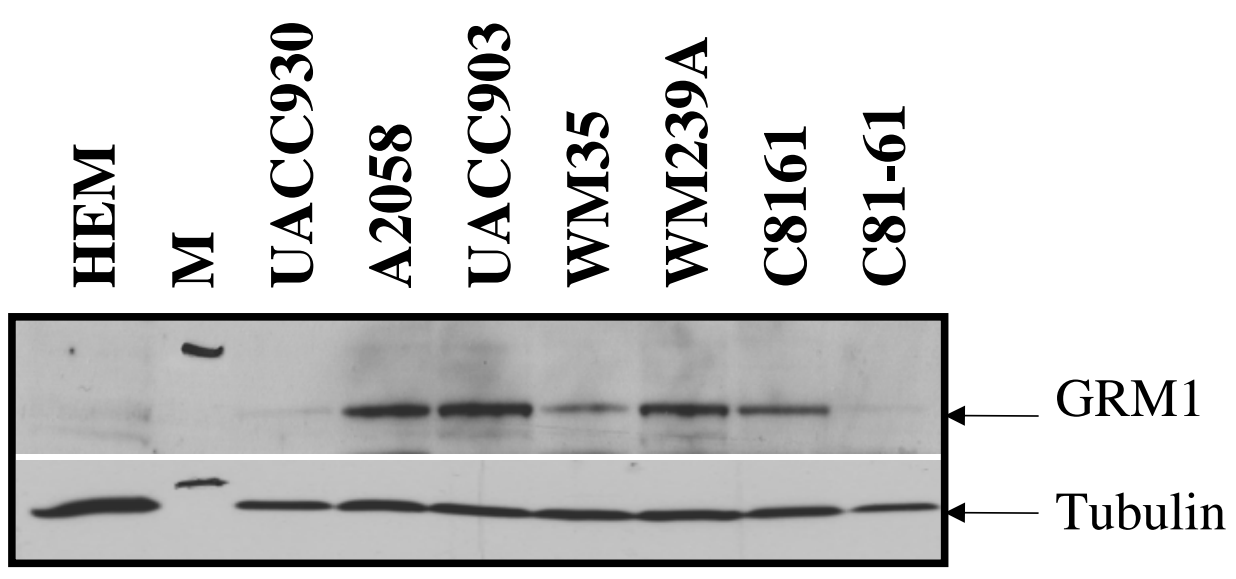

Figure 10

GRM1 Expression in Human Melanoma Cell Lines: Western Blot

GRM1 expression of human melanoma cell lines was examined by western immunoblots. Protein lysates were extracted from cell lines. The top gel showed the expression of GRM1 in these cell lines and the bottom gel showed tubulin expression for the loading control. HEM is normal human epidermal melanocytes; UACC930, A2058, UACC903, WM35, WM239A, C8161 and C81-61 are human melanoma cell lines. UACC930 and HEM were used as negative controls. $\mathrm{M}$ is a protein size marker. Five out of seven human melanoma cell lines shown here had detectable GRM1 protein expression. 


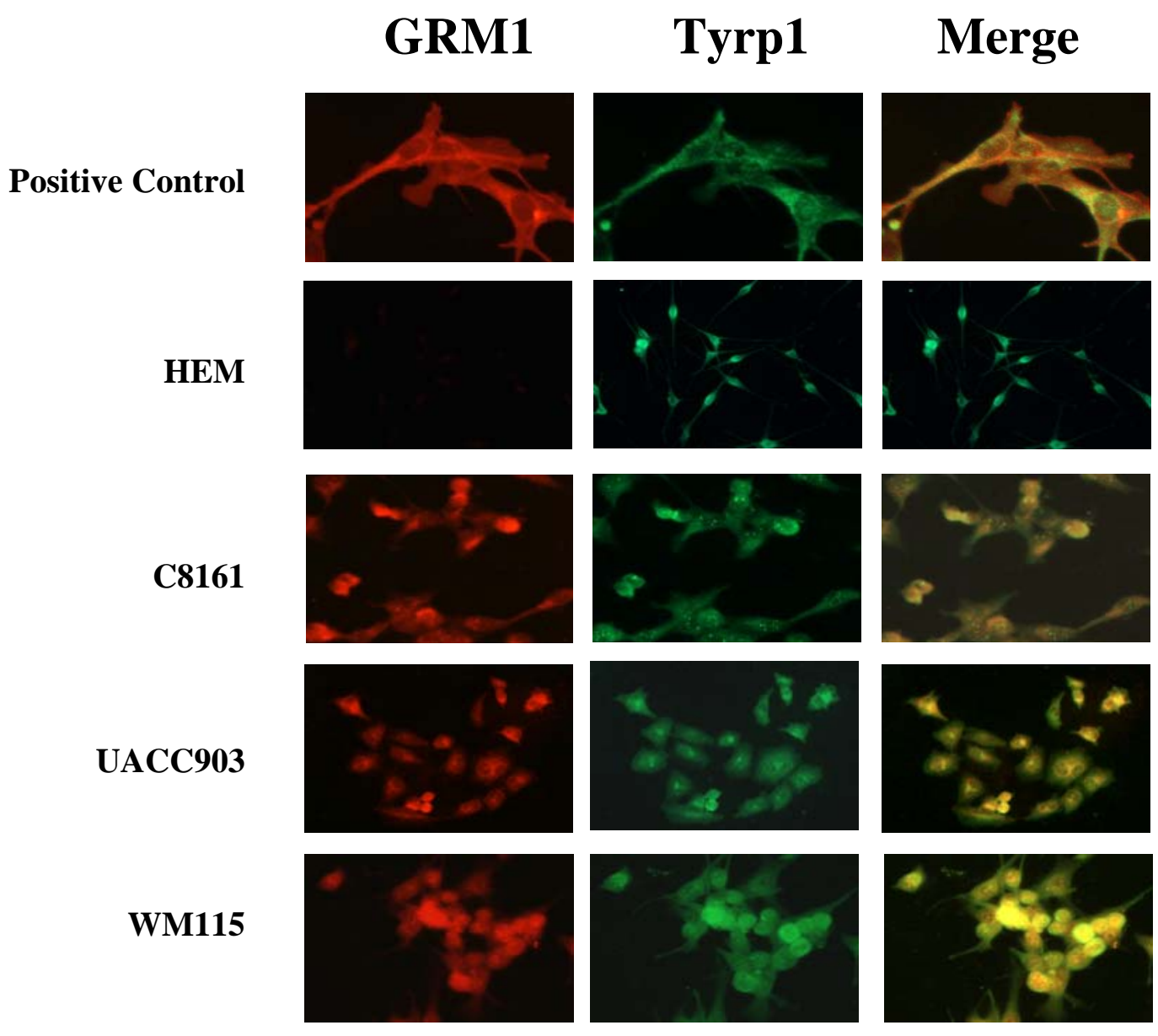

Figure 11

GRM1 Expression in Human Melanoma Cell Lines: Immunofluorescence

GRM1 expression of human melanoma cell lines was examined by coimmunofluorescence. Left column is GRM1 stained cells, middle column is Tyrp1 stained cells and the right column is the merged image of the two. The positive control is a stable clone of normal mouse melanocytes (Melan-A) transfected with Grm1 cDNA. HEM (Human epidermal melanocytes) is used as a normal cell control. C8161, UACC903 and WM115 are human melanoma cell lines. 


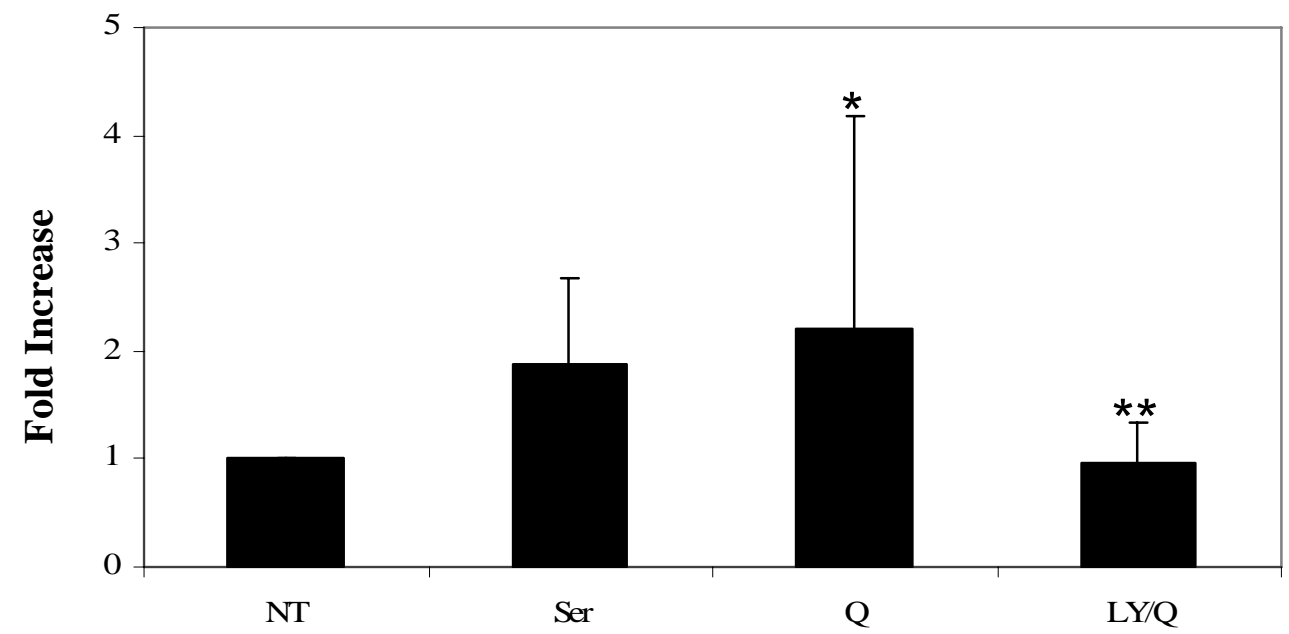

Figure 12

IP3 Accumulation in C8161 Human Melanoma Cell Line

The functionality of GRM1 was examined by GRM1-agonist induced IP3 accumulation in C8161, a human melanoma cell line. Cells were stimulated with Lquisqualate alone $(\mathrm{Q}, 10 \mu \mathrm{M})$ for 15 minutes, or pretreated with LY367385 $(10 \mu \mathrm{M})$ for 15 minutes followed by stimulation with L-quisqualate (LY/Q) for 15 minutes. Data were expressed relative to no treatment (NT). Fetal bovine serum (10\%) was used as a positive control (Ser). Statistically significant elevated levels of accumulated IP3 are detected in Q treated cells. Pretreatment of these cells with specific antagonist of GRM1 LY367385 abolished Q induced IP3 accumulation. An average of three independent experiments is shown; bars, SD; *, $\mathrm{P}<0.05$ compared to NT (t-test); **, $\mathrm{P}<0.05$ compared to $\mathrm{Q}$ (t-test). 


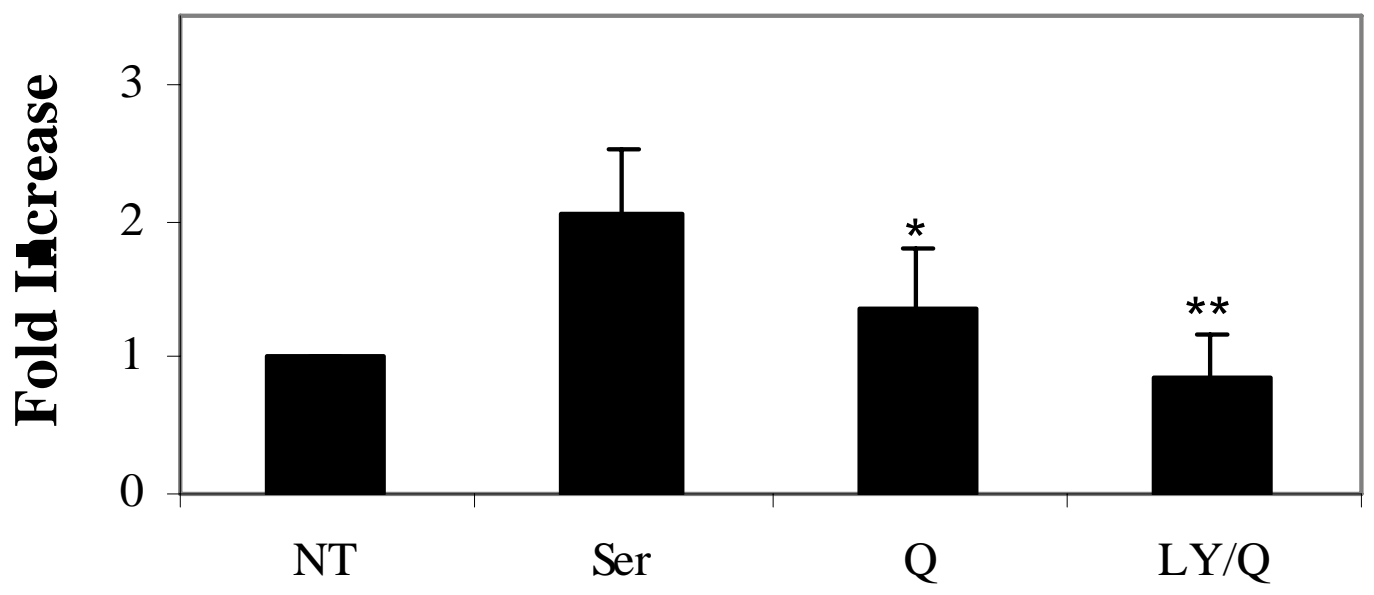

Figure 13

IP3 Accumulation in WM239A Human Melanoma Cell Line

The functionality of GRM1 was examined by GRM1-agonist induced IP3 accumulation in WM239A, a human melanoma cell line. Cells were stimulated with L-quisqualate alone $(\mathrm{Q}, 10 \mu \mathrm{M})$ for 15 minutes, or pretreated with LY367385 $(10 \mu \mathrm{M})$ for 15 minutes followed by stimulation with L-quisqualate (LY/Q) for 15 minutes. Data were expressed relative to no treatment (NT). Fetal bovine serum (10\%) was used as a positive control (Ser). Statistically significant elevated levels of accumulated IP3 are detected in Q treated cells. Pretreatment of these cells with specific antagonist of GRM1 LY367385 abolished Q induced IP3 accumulation. An average of three independent experiments is shown; bars, SD; *, $\mathrm{P}<0.05$ compared to NT (t-test); **, $\mathrm{P}<0.05$ compared to $\mathrm{Q}$ (t-test). 


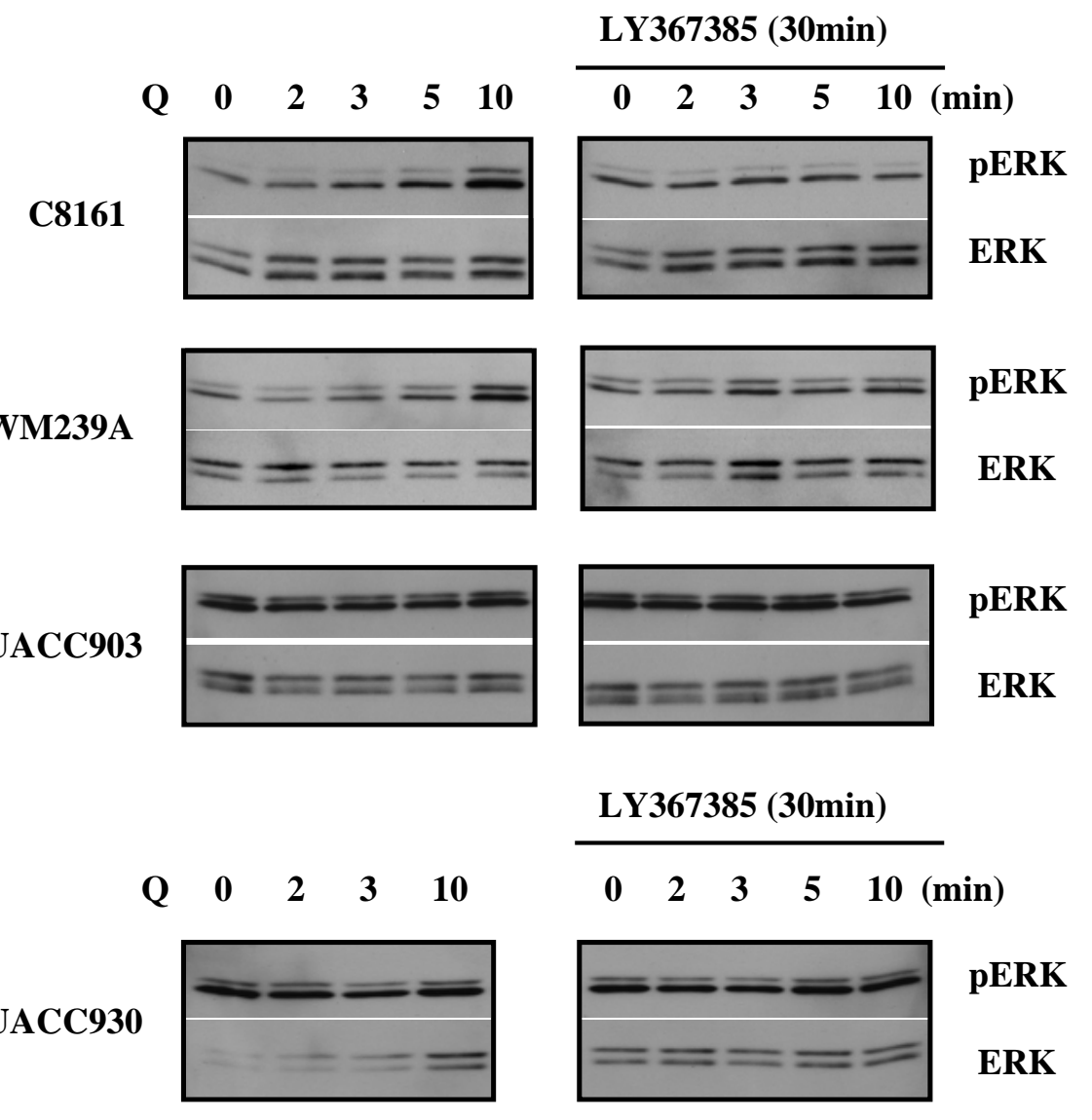

Figure 14

Activation/Inhibition of GRM1 by Agonist (L-Quisqualate) and Antagonist (LY367385)

To demonstrate the functionality of GRM1 in human melanoma cell lines, cells were stimulated with L-quisqualate (Q) for up to 10 minutes. Preincubation of cells with GRM1-specific antagonist, LY367385, for 30 minutes prior to Q induction, led to suppression of ERK phosphorylation in C8161 and WM239A. However, in a human melanoma cell line with the V600E BRAF activating mutation (UACC903), Q induction led to no modulation of ERK phosphorylation with high basal levels of phosphorylated ERK (0 min with Q). In addition, UACC930, a human melanoma cell line with a truncation mutation of GRM1, Q treatment led to no modulation of phosphorylated ERK. The membrane was stripped and re-probed with total ERK for the loading control. 


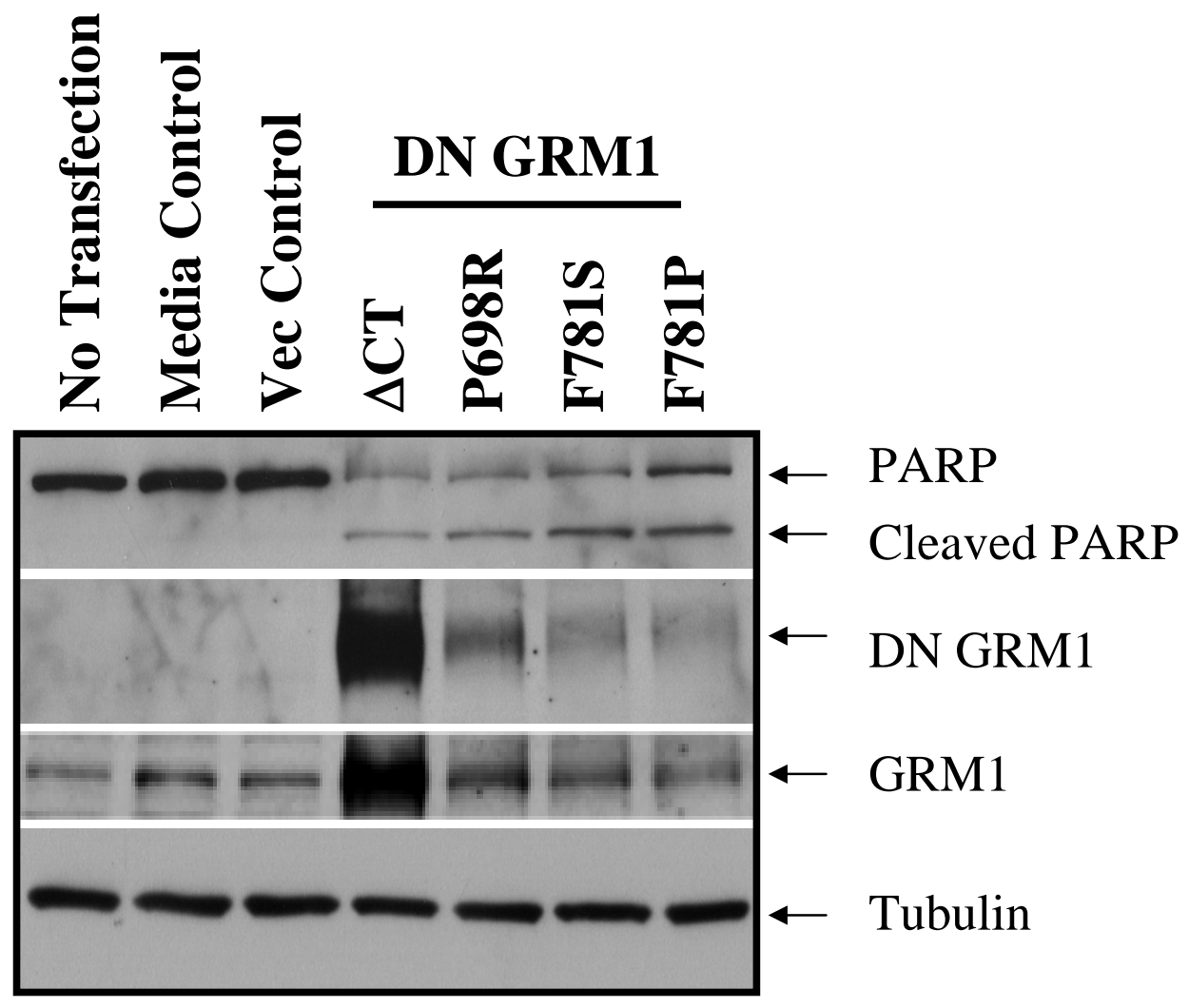

Figure 15

Apoptosis Induction by Dominant Negative Mutants of GRM1 (dnGRM1) in C8161

Dominant negative mutants of GRM1 (dnGRM1) induced apoptosis in C8161 human melanoma cell line. C8161 human melanoma cells were transfected with vector control or dnGRM1s. Apoptosis was assessed by the presence of the cleaved form of PARP at $89 \mathrm{kDa}$ at 24 hours post-transfection. Three independent experiments were done and a representative blot is shown here. Grm1 antibody that only recognizes the rodent form of Grm1 was used to show the levels of transfected GRM1 (dnGRM1). The membrane was stripped and reprobed with GRM1 antibody that recognized both endogenous (human) and transfected GRM1 (GRM1). Negative controls included no transfection and media control, where growth media were changed to OptiMEM for 6 hours during the transfection. $\alpha$-Tubulin was used as a loading control. Apoptosis was only observed in cell lines transfected with dominant negative mutants of GRM1. 


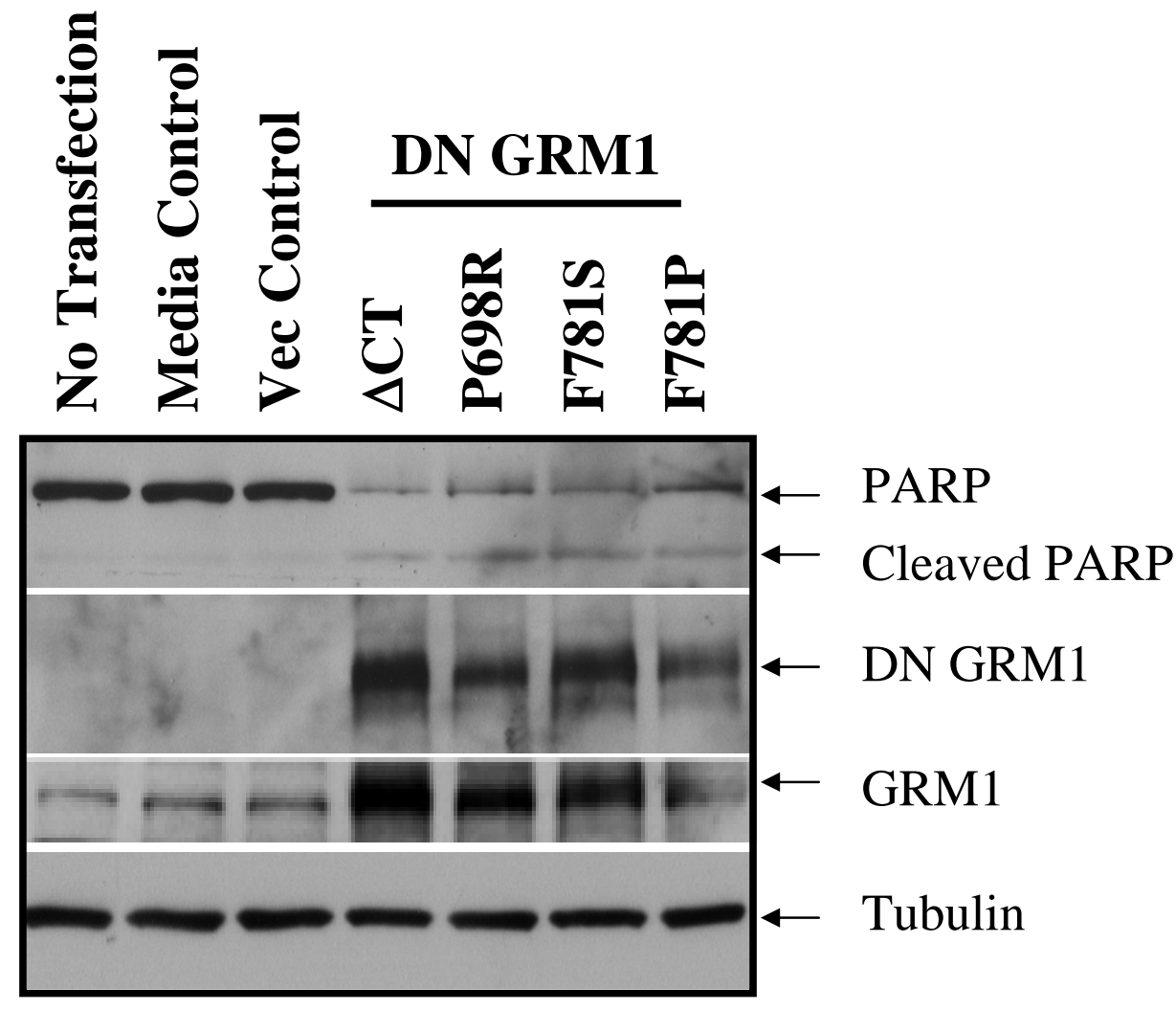

Figure 16

Apoptosis Induction by Dominant Negative Mutants of GRM1 (dnGRM1) in WM239A

Dominant negative mutants of GRM1 (dnGRM1) induced apoptosis in WM239A human melanoma cell line. Human melanoma cells were transfected with vector control or dnGRM1s. Apoptosis was assessed by the presence of the cleaved form of PARP at $89 \mathrm{kDa}$ at 24 hours post-transfection. Three independent experiments were done and a representative blot is shown here. Grm1 antibody that only recognizes the rodent form of Grm1 was used to show the levels of transfected GRM1 (dnGRM1). The membrane was stripped and reprobed with GRM1 antibody that recognized both endogenous (human) and transfected GRM1 (GRM1). Negative controls included no transfection and media control, where growth media were changed to OptiMEM for 6 hours during the transfection. $\alpha$-Tubulin was used as a loading control. Apoptosis was only observed in cell lines transfected with dominant negative mutants of GRM1. 


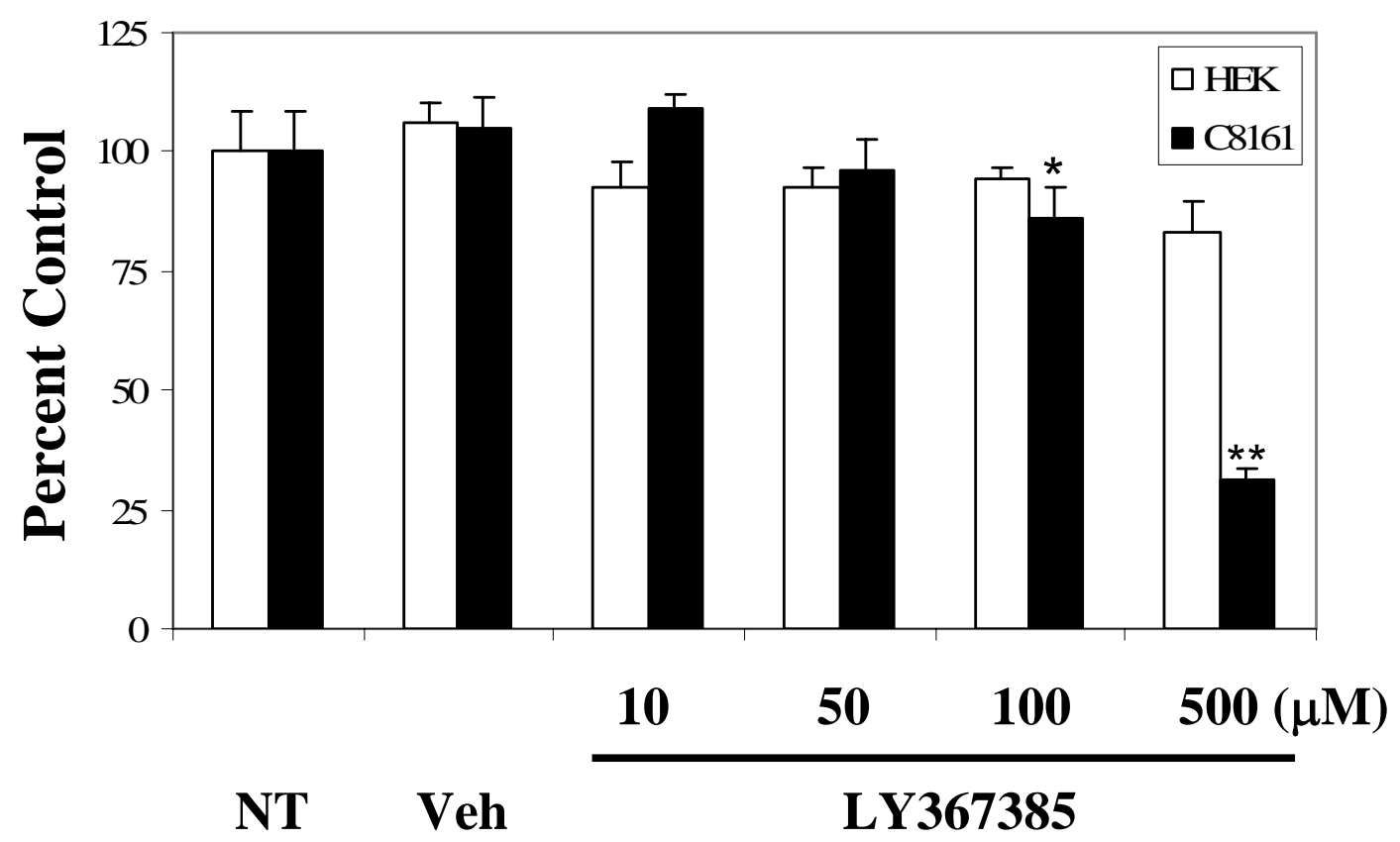

Figure 17

MTT Cell Proliferation Assay pf C8161 and HEK293A Cells Treated with a GRM1antagonist, LY367385

Biological responses of human melanoma cells to LY367385 were measured. Cells were treated with LY367385 at concentrations of 10, 50, 100 and $500 \mu \mathrm{M}$ respectively. No treatment (NT) and $500 \mu \mathrm{M} \mathrm{NaOH}$ (Veh) were used as controls. Measurement of cell viability/growth was carried out by MTT assays for four days. Each point represents the average of quadruplicates. Minimum of three independent experiments were done and a representative graph is shown here. Only measurements on day 4 are shown here. LY367385 suppressed growth of C8161 human melanoma cells (black bar) in a dose-dependent manner, but had no significant effect on HEK cells (white bar). Bar, SD; *, P $<0.05$ compared to HEK (t-test); **, $\mathrm{P}<0.001$ compared to HEK (t-test). 
NT Veh LY367385
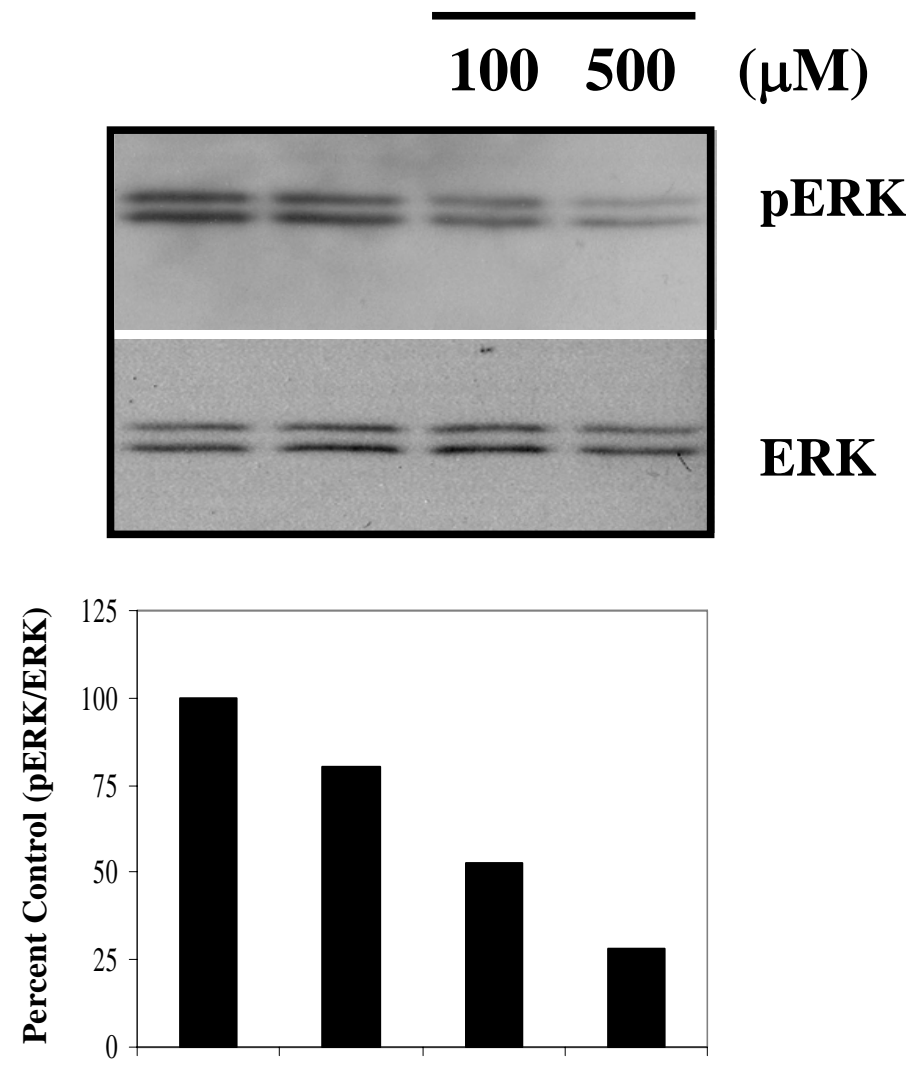

Figure 18

Suppression of ERK Activation by LY367385 Treatment

The status of ERK phosphorylation after treatment with LY367385 was examined by immunoblots at day 4. C8161 cells were not treated (NT), or treated with 100 or 500 $\mu \mathrm{M}$ LY367385 or with $500 \mu \mathrm{M} \mathrm{NaOH}$ (Veh). Protein lysates were prepared for western immunoblots and probed with phospho-ERK. The same membranes were stripped and reprobed with total ERK. Quantitation is shown at the bottom as a graph using NT as the control (100\%). 


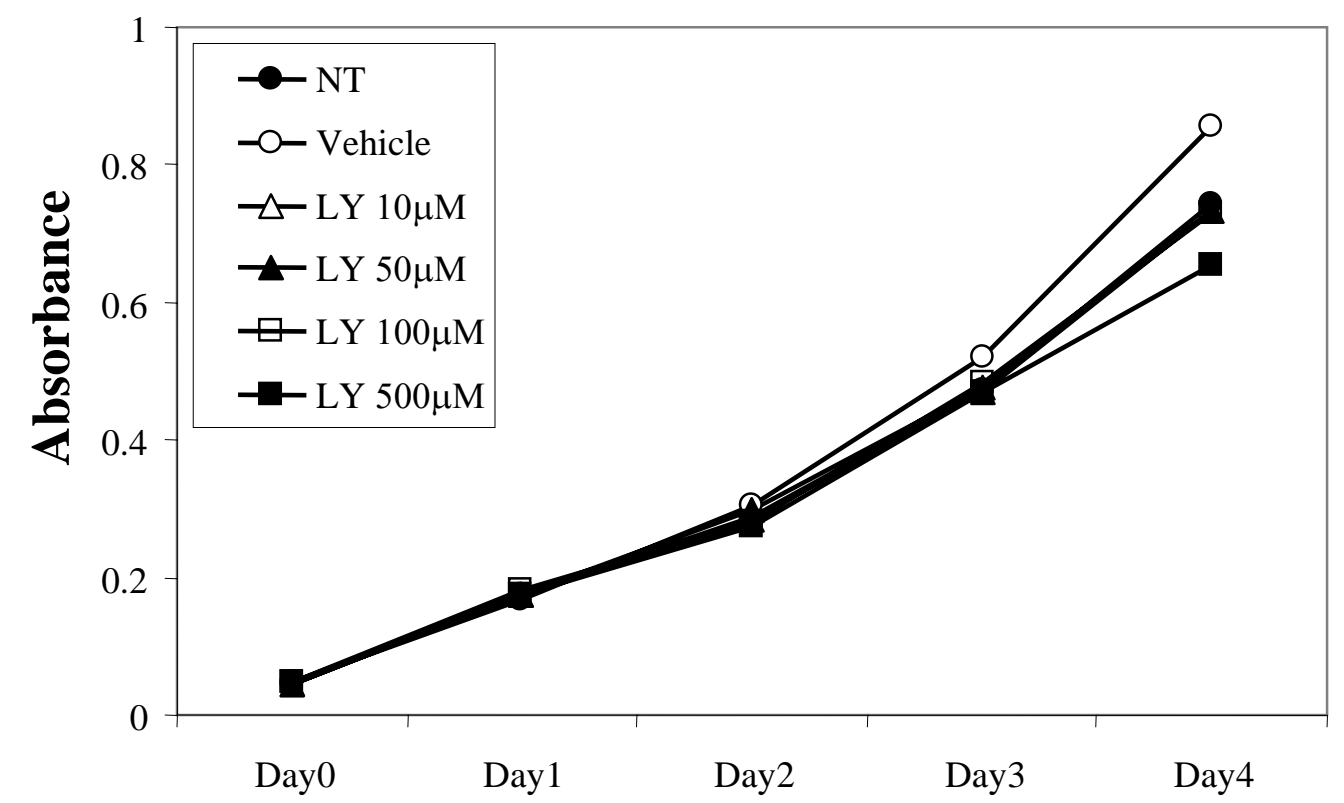

Figure 19

MTT Cell Proliferation Assay of WM239A Treated with LY367385

The biological response of WM239A human melanoma cells to LY367385 was measured. Cells were treated with LY367385 at concentrations of 10, 50, 100 and 500 $\mu \mathrm{M}$. No treatment (NT) and $500 \mu \mathrm{M} \mathrm{NaOH}$ (Vehicle) were used as controls. Each point represents the average of quadruplicates. Minimum of three independent experiments were done and a representative graph is shown here. Measurement of cell viability/growth was carried out by MTT assays for four days. $500 \mu \mathrm{M}$ LY367395 had only $12 \%$ growth inhibitory effect on WM239A cells. 


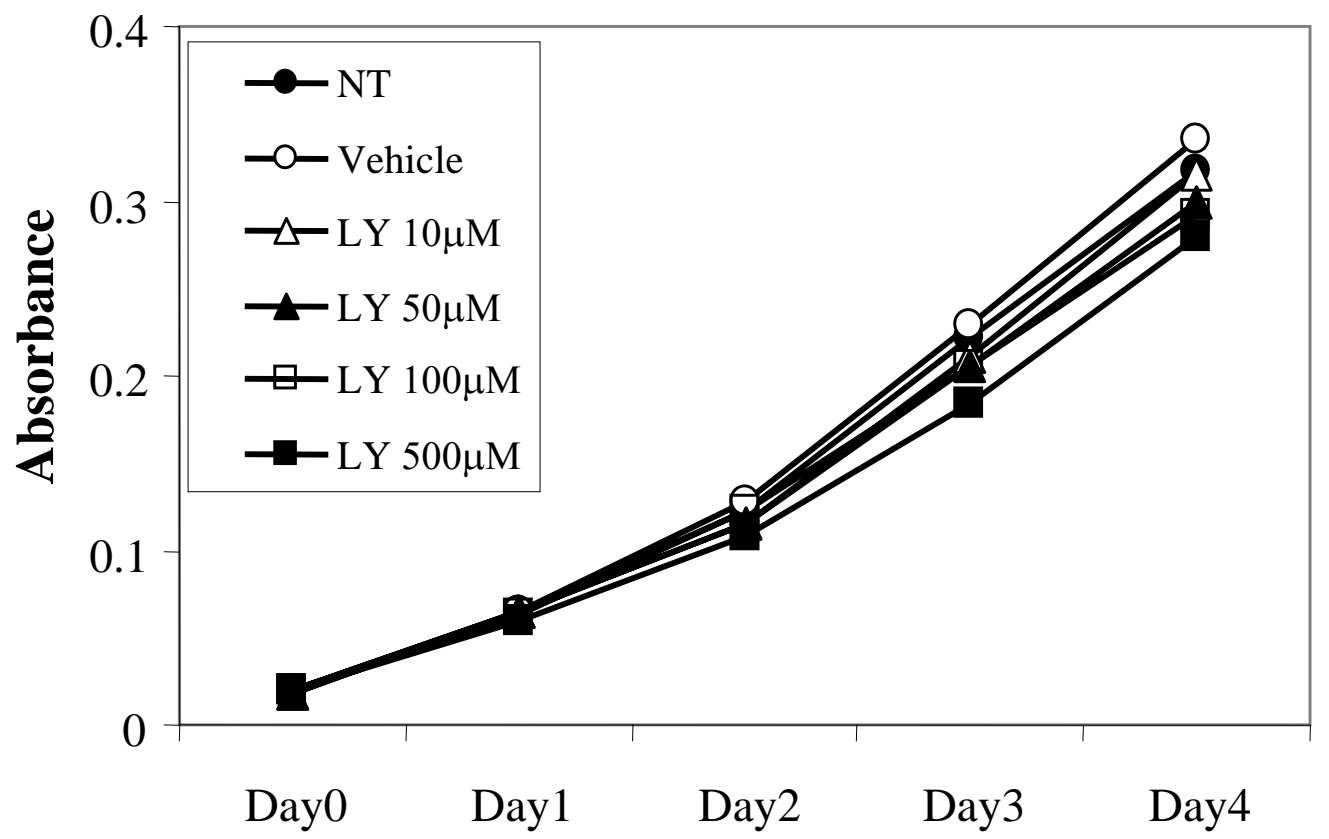

Figure 20

MTT Cell Proliferation Assay of UACC903 Treated with LY367385

The biological response of UACC903 human melanoma cells to LY367385 was measured. UACC903 bears BRAF activating mutation. Cells were treated with LY367385 at concentrations of 10, 50, 100 and $500 \mu \mathrm{M}$. No treatment (NT) and 500 $\mu \mathrm{M} \mathrm{NaOH}$ (Vehicle) were used as controls. Each point represents the average of quadruplicates. Minimum of three independent experiments were done and a representative graph is shown here. Measurement of cell viability/growth was carried out by MTT assays for four days. $500 \mu \mathrm{M}$ LY367395 had only 12\% growth inhibitory effect on UACC903 cells. 


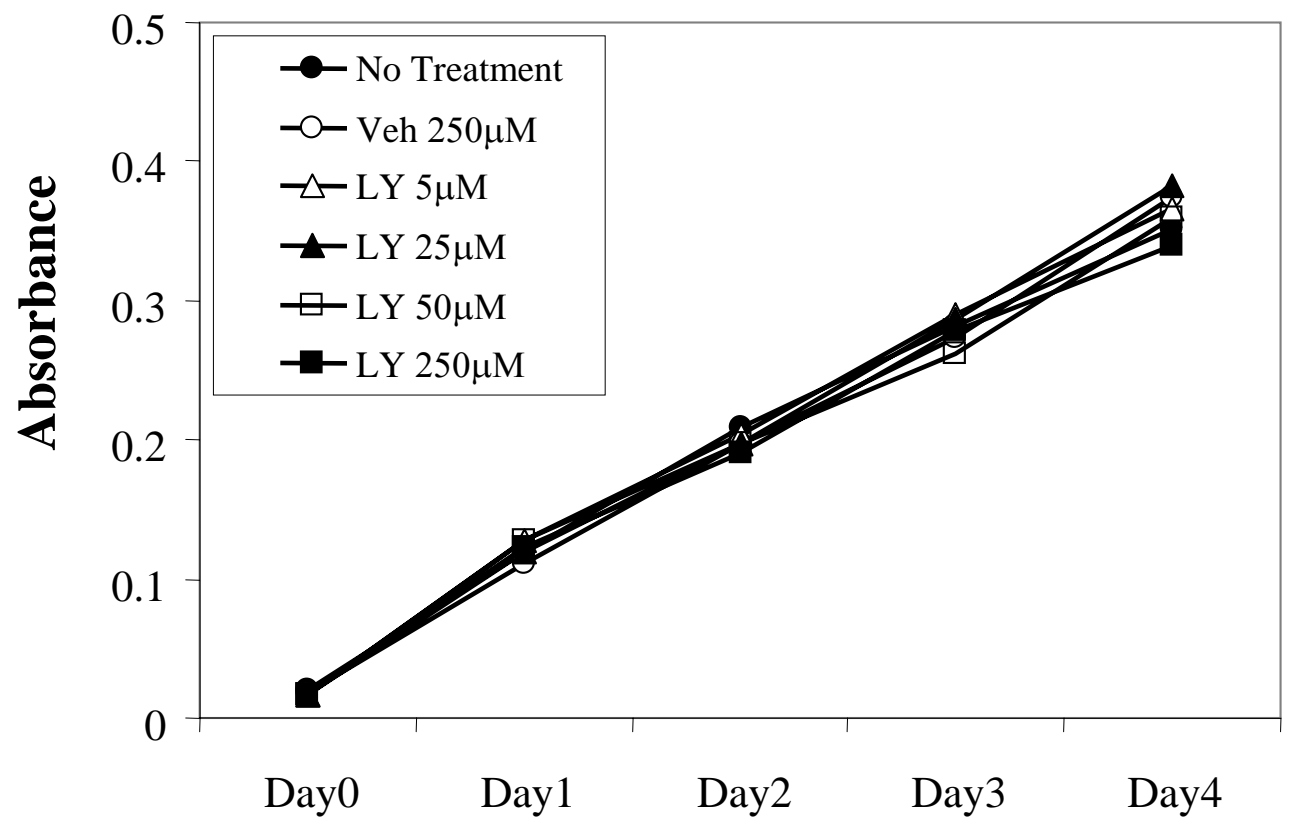

Figure 21

MTT Cell Proliferation Assay of WM115 Treated with LY367385

Biological responses of human melanoma cells to LY367385 were measured on WM115 human melanoma cell line. WM115 bears BRAF activating mutation. Cells were treated with LY367385 at concentrations of 5, 25, 50 and $250 \mu \mathrm{M}$ respectively. No treatment (NT) and $250 \mu \mathrm{M} \mathrm{NaOH}$ (Vehicle) were used as controls. Each point represents the average of quadruplicates. Measurement of cell viability/growth was carried out by MTT assays for four days. LY367395 had little or no growth inhibitory effect on WM115 cells. 


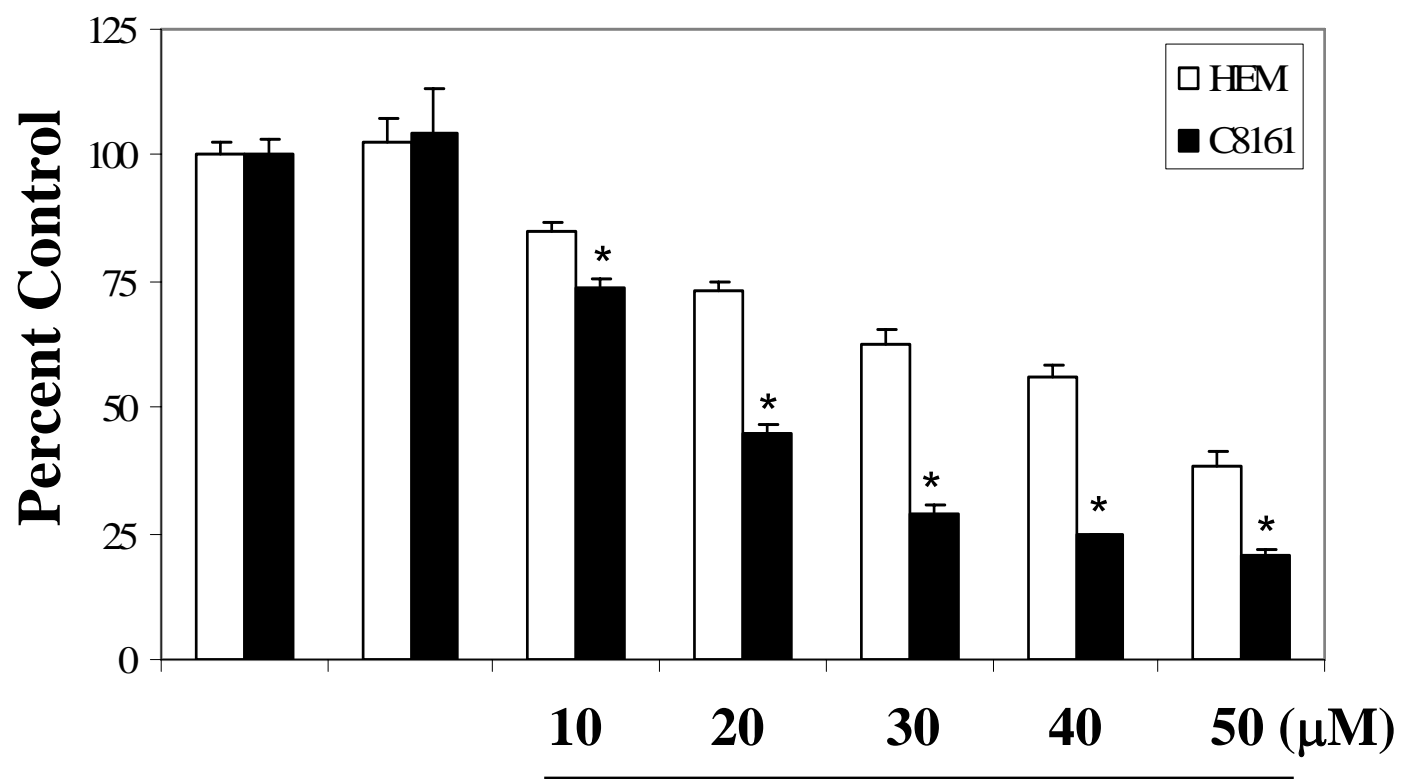

NT Veh BAY 36-7620

Figure 22

MTT Cell Proliferation Assay of C8161 and HEM Cells Treated with a GRM1antagonist, BAY 36-7620

The biological consequence of BAY36-7620 treatments in HEM (white bar) or C8161 (black bar) cells was measured by MTT cell viability assays. Cells were either not treated (NT), vehicle treated (Veh), or treated with 10, 20, 30, 40 or $50 \mu \mathrm{M}$ of BAY 36-7620 respectively for four days. Each point represents the average of quadruplicates. Minimum of three independent experiments were done and a representative graph is shown here. In the presence of BAY36-7620, the growth of C8161 cells was suppressed in a dose-dependent manner, while vehicle treatment had very little or no effect on cell growth. Only measurements on day 4 are shown here. HEM cells were less sensitive to BAY 36-7620 in comparison to C8161 cells. Bar, $\mathrm{SD} ;{ }^{*}, \mathrm{P}<0.001$ compared to HEM (t-test). 


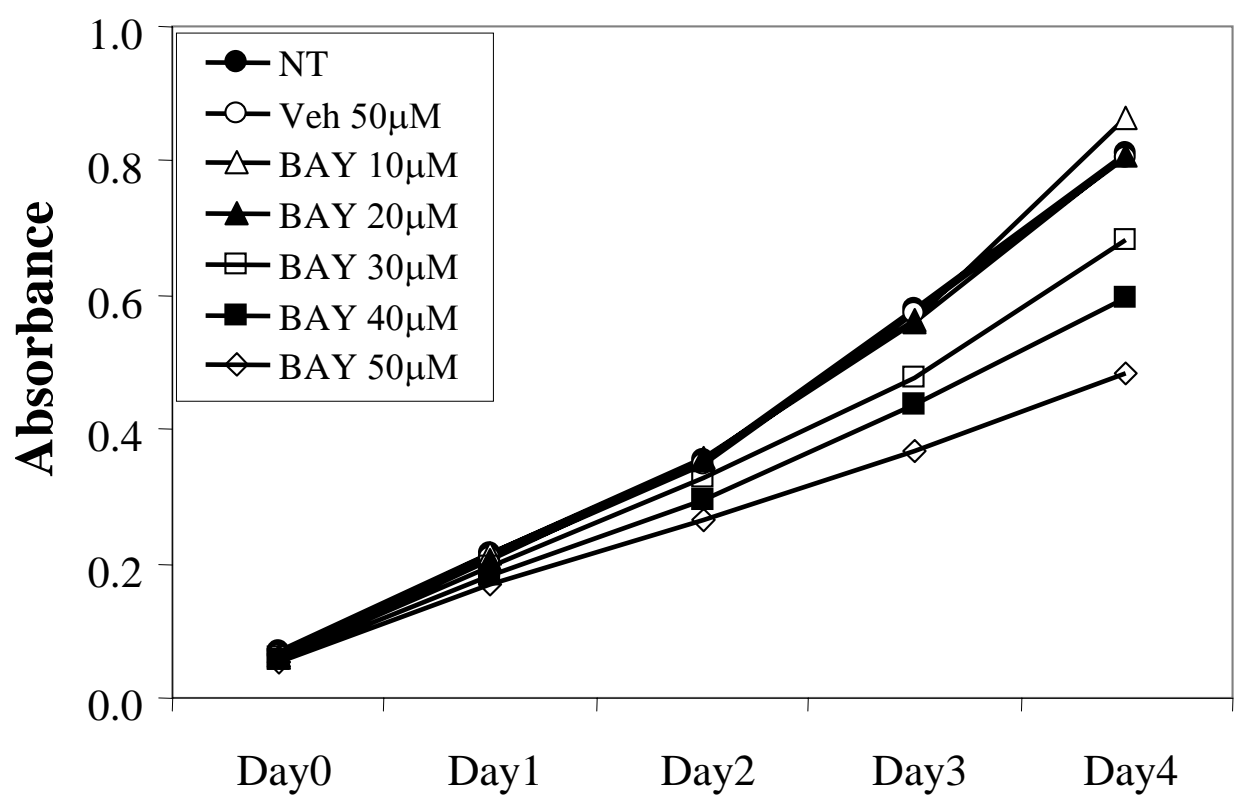

Figure 23

MTT Cell Proliferation Assay of WM239A Treated with BAY 36-7620

Biological consequences of BAY 36-7620 treatments in WM239A cells were measured by MTT cell viability assays. Cells were either not treated (NT), vehicle treated (Veh), or treated with 10, 20, 30, 40 or $50 \mu \mathrm{M}$ of BAY 36-7620 respectively for four days. Each point represents the average of quadruplicates. Minimum of three independent experiments were done and a representative graph is shown here. In the presence of BAY 36-7620, the growth of WM239A cells was suppressed in a dosedependent manner, while vehicle treatment had very little or no effect on cell growth. 


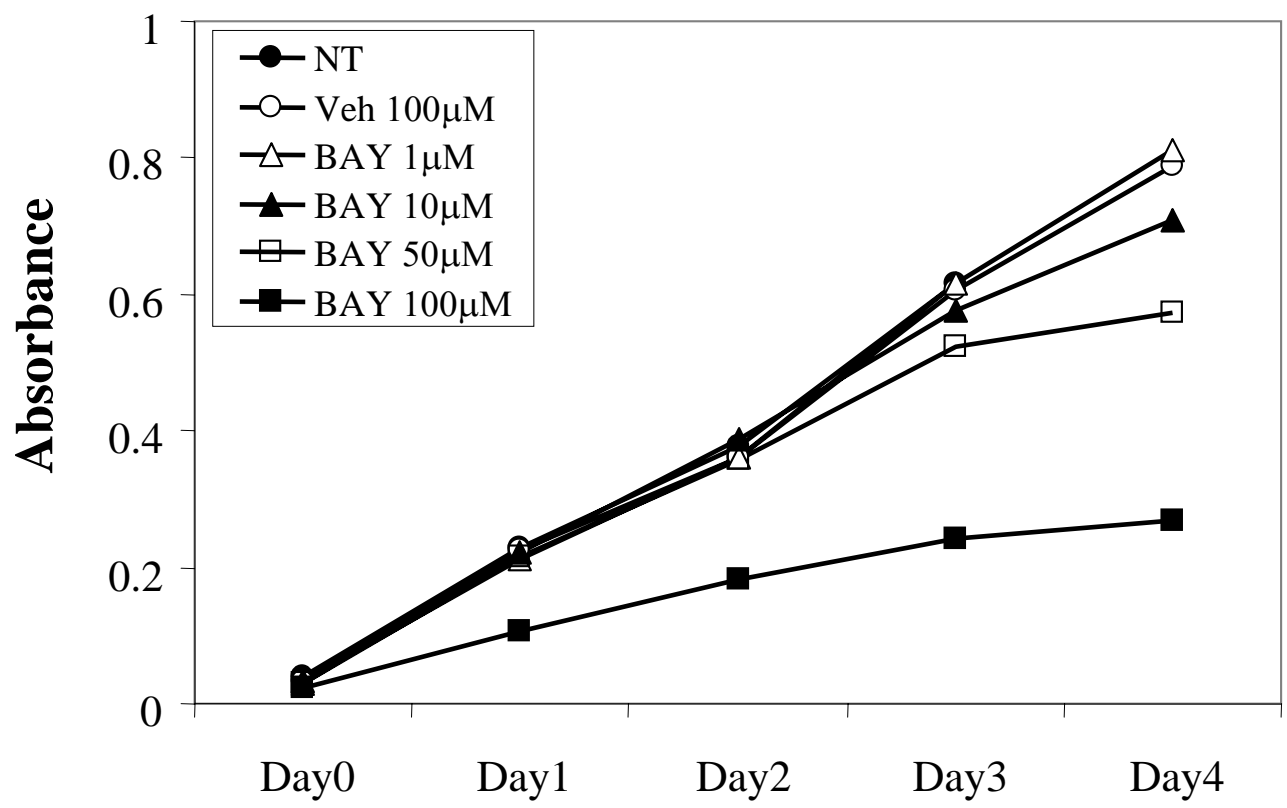

Figure 24

MTT Cell Proliferation Assay of UACC903 Treated with BAY 36-7620

Biological consequences of BAY 36-7620 treatments in UACC903 cells were measured by MTT cell viability assays. Cells were either not treated (NT), vehicle treated (Veh), or treated with $1,10,50$ or $100 \mu \mathrm{M}$ of BAY 36-7620 respectively for four days. Each point represents the average of quadruplicates. Minimum of three independent experiments were done and a representative graph is shown here. In the presence of BAY36-7620, the growth of UACC903 cells was suppressed only at 100 $\mu \mathrm{M}$, while vehicle treatment had very little or no effect on cell growth. 
24 Hours

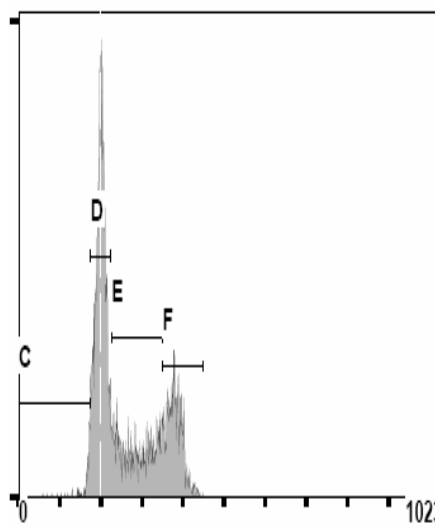

No Treatments

48 Hours

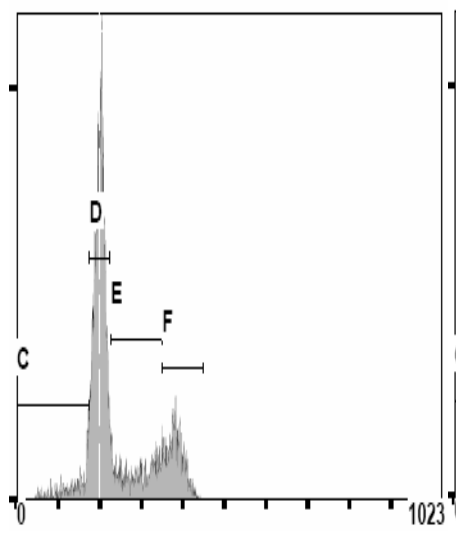

No Treatments
Vehicle

BAY36-7620 100 $\mu \mathrm{M}$

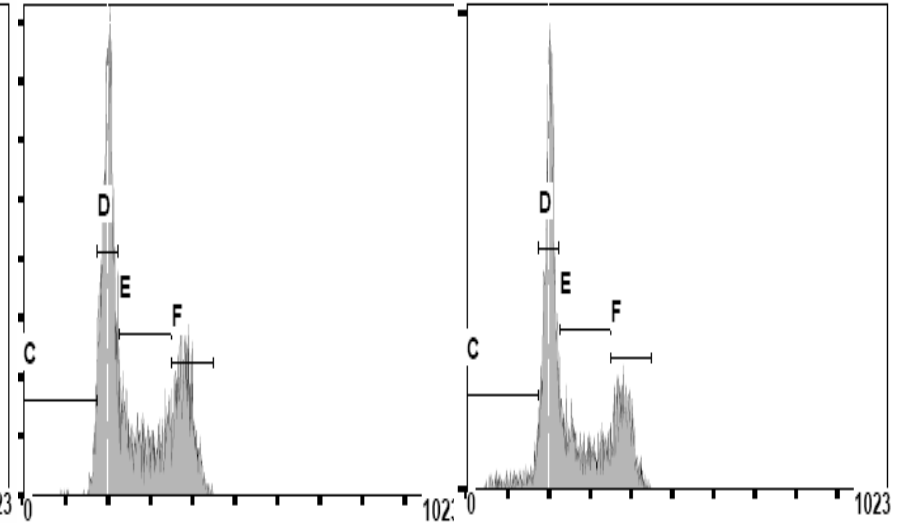

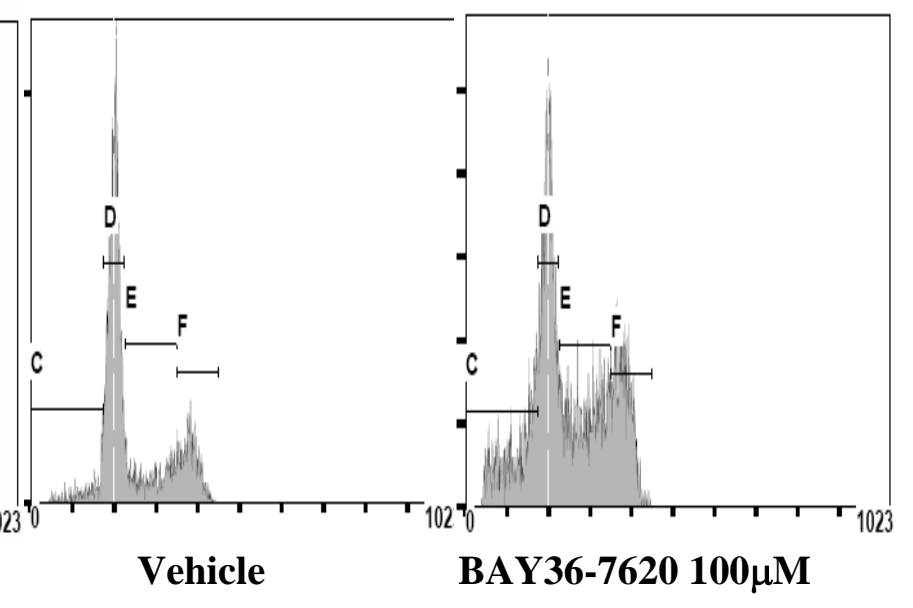

Figure 25

Cell Cycle Analysis of BAY 36-7620 treated C8161

Cell cycle analysis were performed with C8161 cells treated with BAY36-7620 at 24 (top) and 48 (bottom) hours. At 24 and 48 hours post-treatment, cells were accumulated in the sub-G1 phase of the cell cycle. DMSO treatment (Vehicle) had little or no effects. Each phase of cell cycle was indicated as C (sub-G1), D (G1), E (S) and F (G2/M) and the percentage of cells in each phase was given in Table 2 (24 hours) and Table 3 (48 hours). 


\section{WM239A}

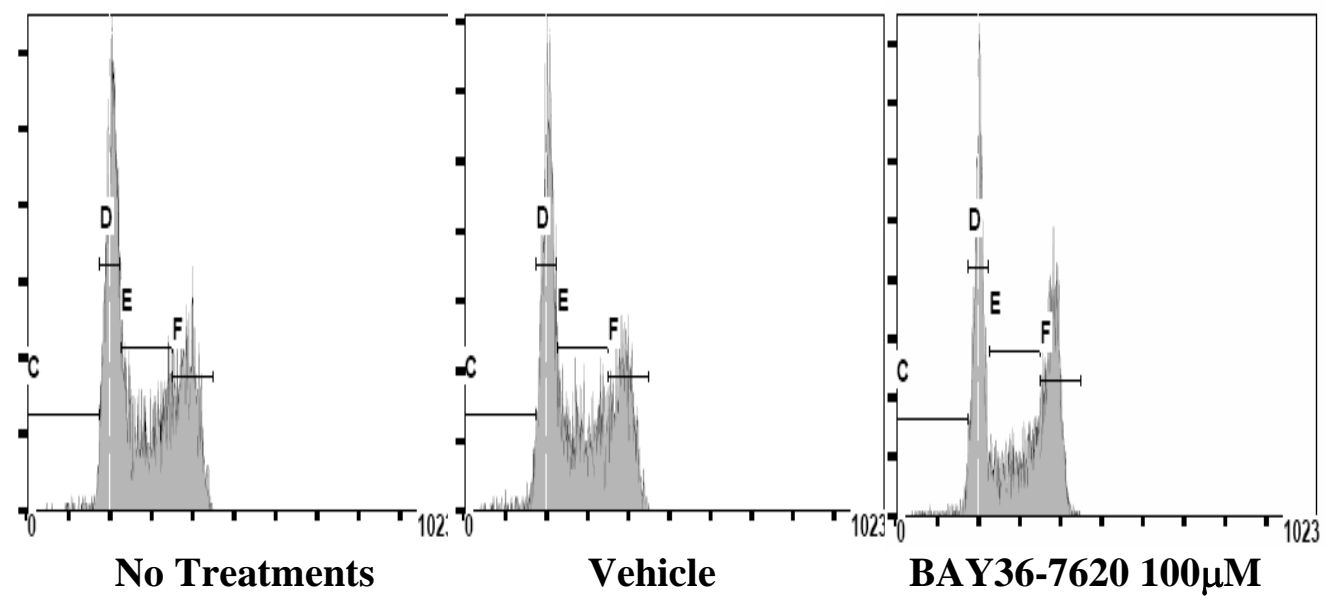

Figure 26

Cell Cycle Analysis of BAY 36-7620 treated WM239A

Cell cycle analysis were performed with WM239A cells treated with BAY 36-7620 at 24 hours. At 24 hours post-treatment, cells were accumulated in the G2/M phase of the cell cycle. DMSO treatment (Vehicle) had little or no effects. Each phase of cell cycle was indicated as C (sub-G1), D (G1), E (S) and F (G2/M) and the percentage of cells in each phase was given in Table 6. 
24 Hours

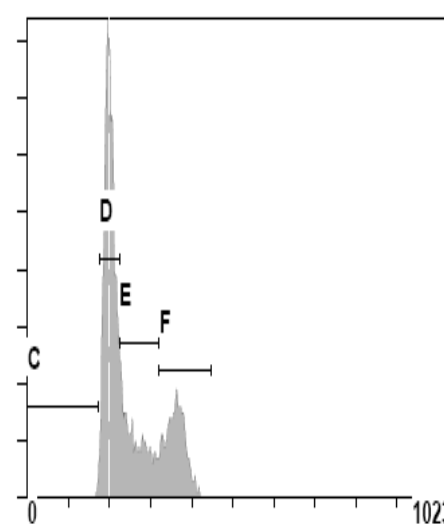

No Treatments

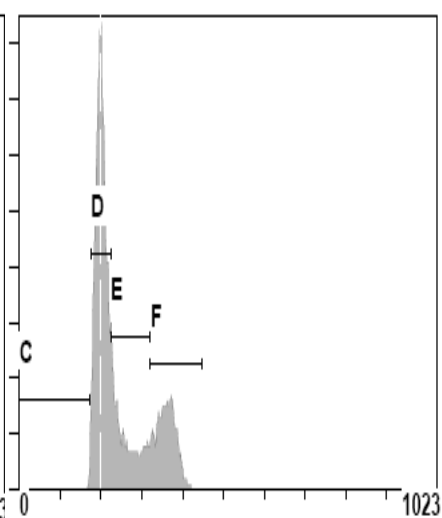

Vehicle

48 Hours

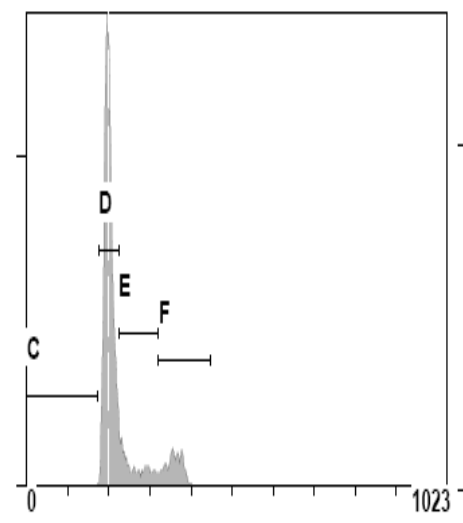

No Treatments

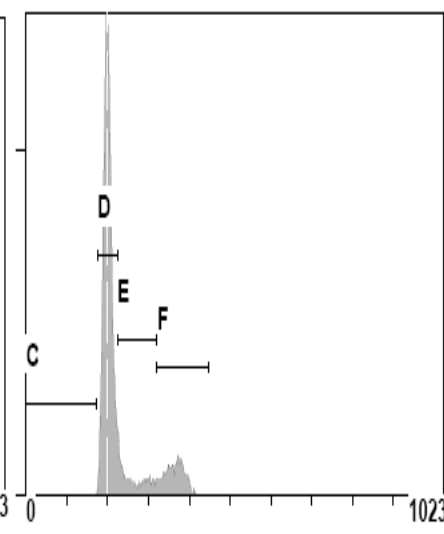

Vehicle

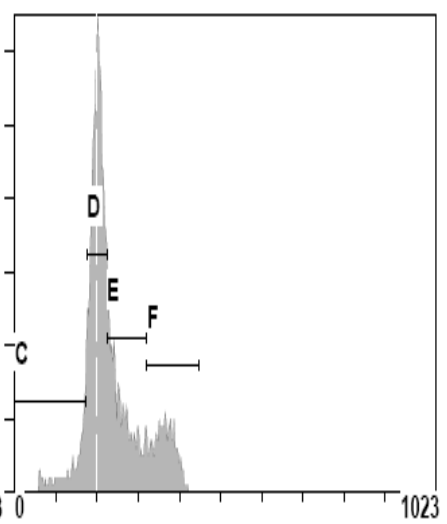

BAY36-7620 100 $\mu M$

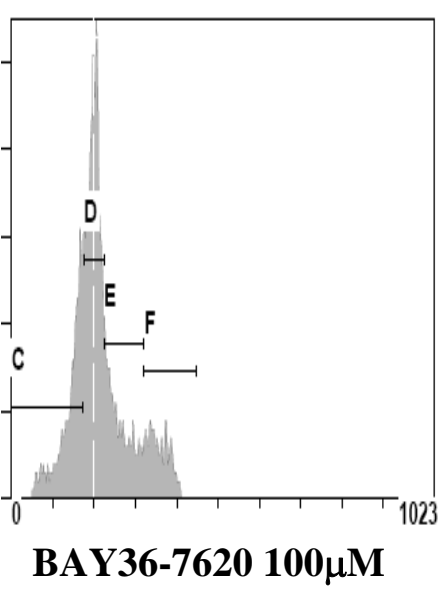

Figure 27

Cell Cycle Analysis of BAY 36-7620 treated UACC903

Cell cycle analysis were performed with UACC903 cells treated with BAY 36-7620 at 24 (top) and 48 (bottom) hours. At 24 and 48 hours post-treatment, cells were accumulated in the sub-G1 and S phases of the cell cycle. DMSO treatment (Vehicle) had little or no effects. Each phase of cell cycle was indicated as C (sub-G1), D (G1), $\mathrm{E}(\mathrm{S})$ and $\mathrm{F}(\mathrm{G} 2 / \mathrm{M})$ and the percentage of cells in each phase was given in Table 8 (24 hours) and Table 9 (48 hours). 


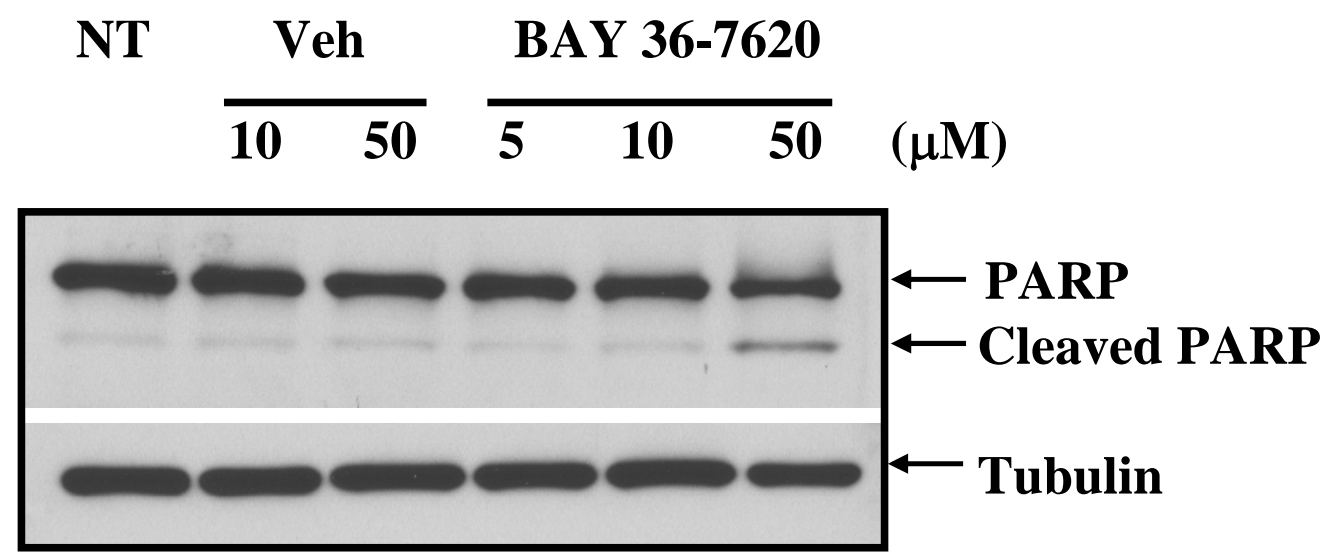

Figure 28

Induction of Apoptosis by BAY 36-7620 Treatments in C8161

Levels of cleaved PARP were examined by western immunoblots in C8161 treated with 10,25 or $50 \mu \mathrm{M}$ BAY 36-7620 for 48 hours. Three independent experiments were done and a representative blot is shown here. Elevated levels of cleaved PARP were only observed in C8161 cells treated with $50 \mu \mathrm{M}$ BAY 36-7620 compared to DMSO treated (Veh) or no treatment (NT). The same blot was probed with $\alpha$-Tubulin to show equal loading. 


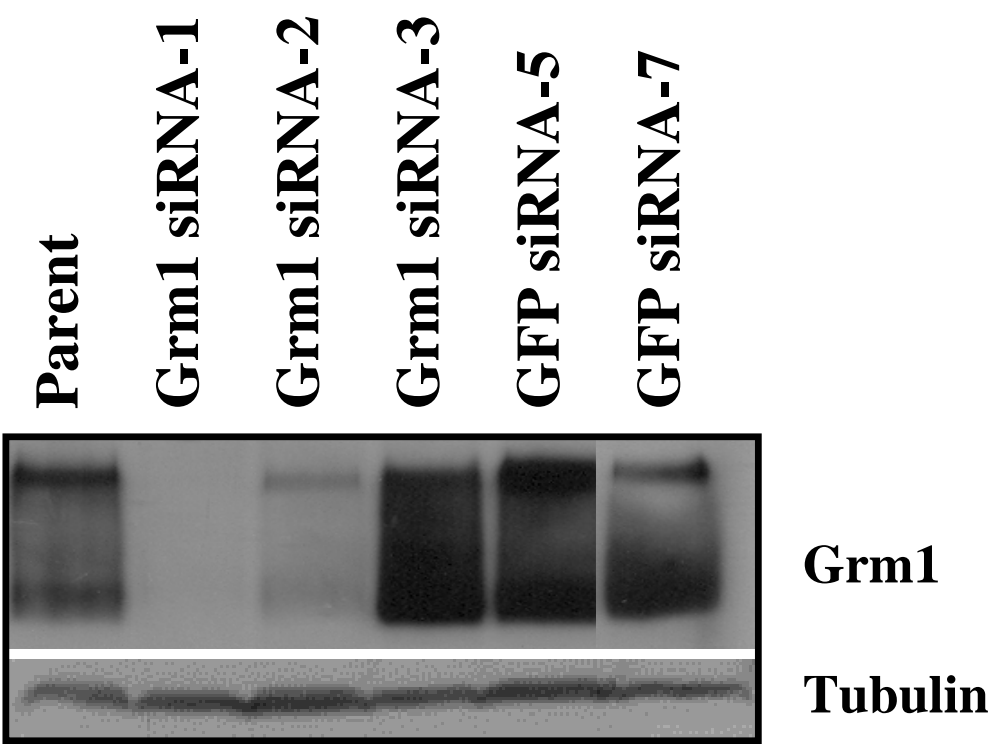

\section{$\begin{array}{llllll}1 & 2 & 3 & 4 & 5 & 6\end{array}$}

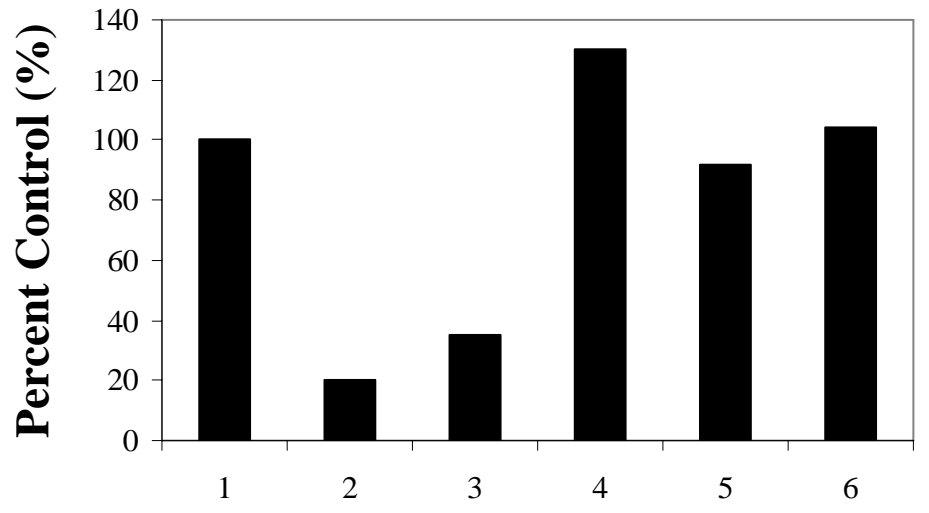

Figure 29

Grm1 siRNA Stable Clones

In order to assess the efficiency of Grm1 siRNA, Grm1 transfected into normal mouse melanocytes (Melan-A) was used (MelanA-mGluR1-3-1). Cells were transfected with Grm1 siRNA or GFP siRNA (control) and stable clones were isolated. Protein lysates were extracted and western immunoblots were performed to measure the efficiency (Top). Grm1 protein expression was reduced up to $80 \%$. Quantitation is shown (Bottom). 


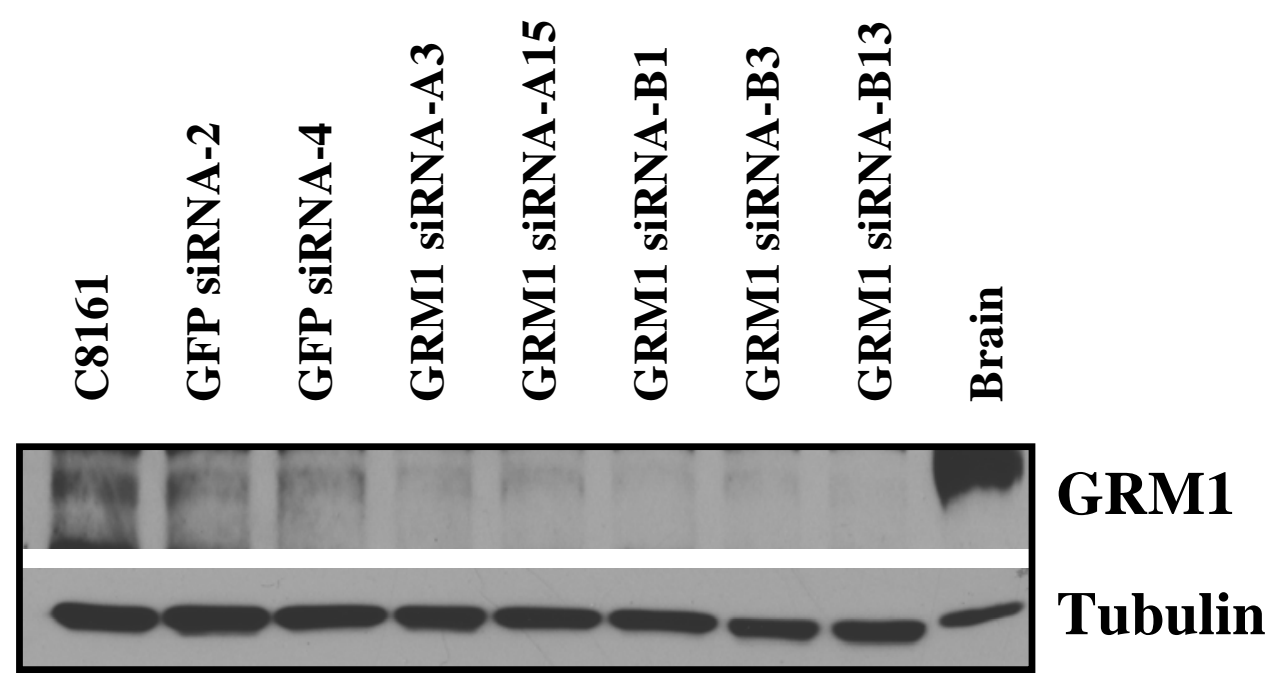

Figure 30

Western Blots of C8161-GRM1 siRNA Stable Clones

C8161 cells were transfected with GRM1 siRNA-A, B or GFP siRNA (control) and stable clones were selected. Protein lysates were extracted and $50 \mu$ g of lysates were loaded on western immunoblots to show GRM1 protein expression (Top). Compared to parent clones or GFP siRNA controls, GRM1 siRNA transfected cells had reduced expression of GRM1. Tubulin was used as a loading control. $5 \mu \mathrm{g}$ of brain extract was used as positive control for GRM1 expression. 

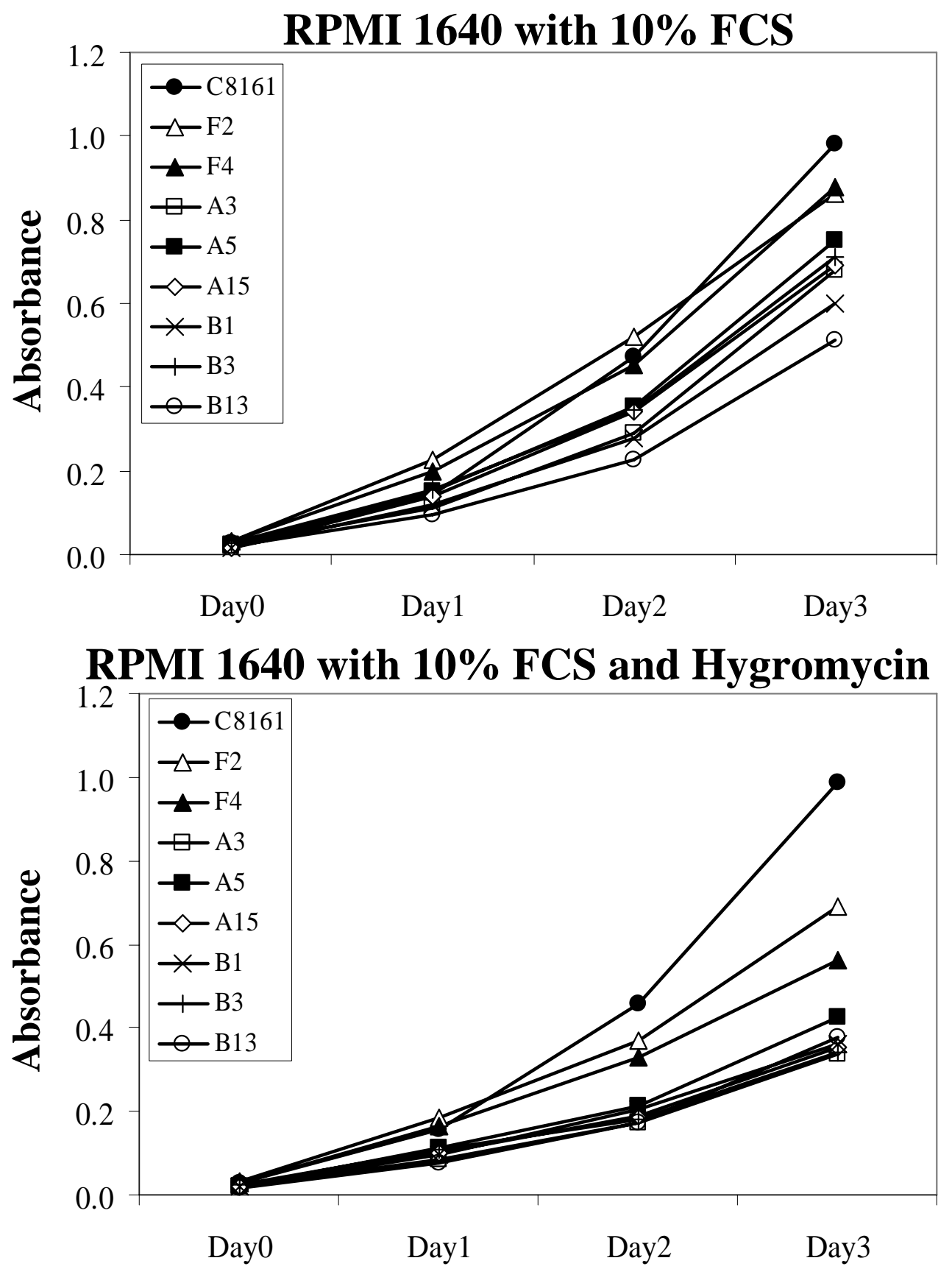

Figure 31

C8161-GRM1 siRNA Stable Clones: MTT Cell Proliferation Assay

MTT assay was performed on stable C8161 clones made with transfected with GRM1 siRNA-A (A), B (B) or GFP (F) siRNA (control) without hygromycin (Top) or with hygromycin (Bottom). Each point represents the average of quadruplicates. Cells transfected with GRM1 siRNA show reduction in cell proliferation compared to cells transfected with GFP siRNA. 

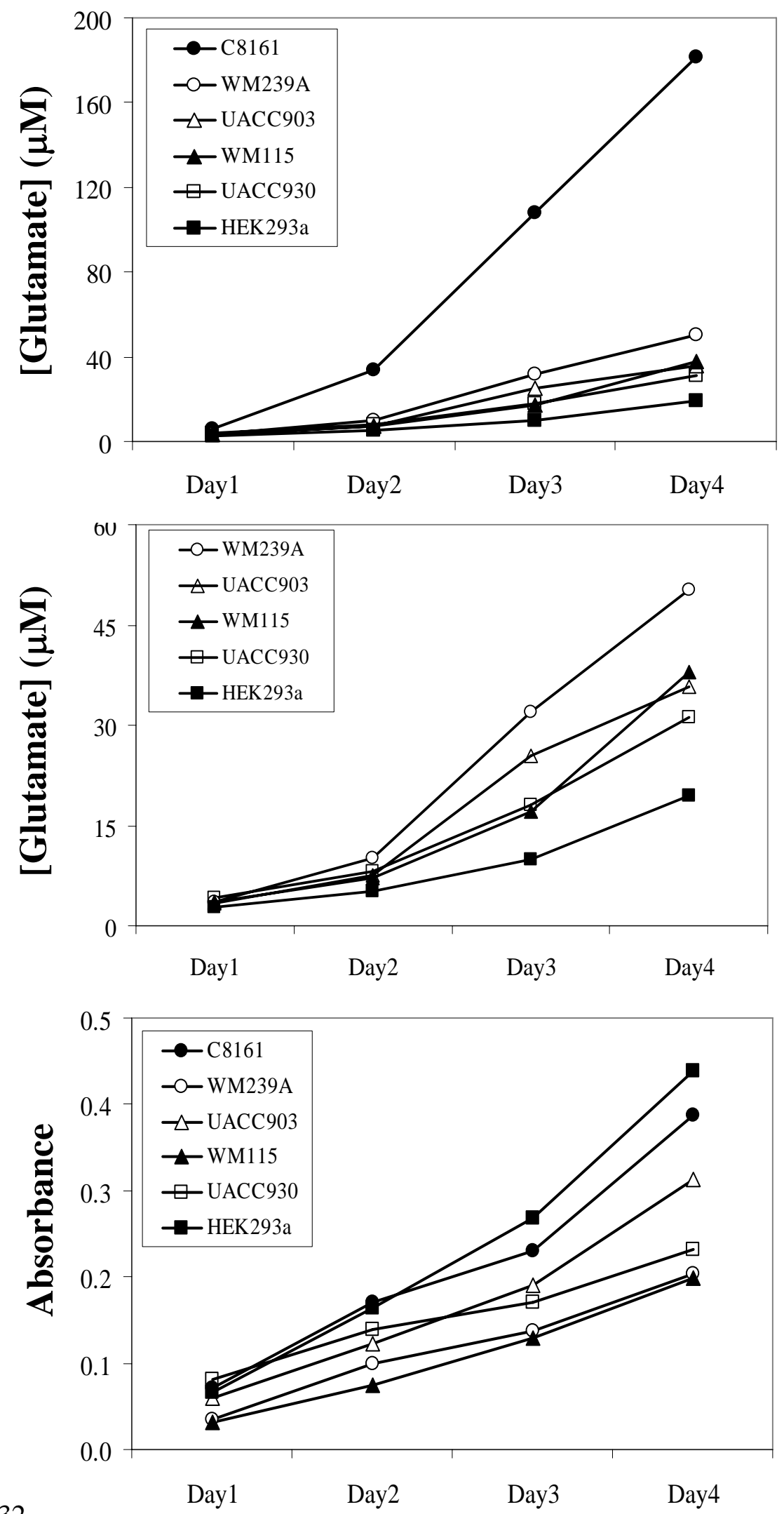

Figure 32 
Figure 32

Glutamate Release Measurements of Human Melanoma Cell Lines

Human melanoma cell lines and HEK cells were examined for levels of released glutamate. Cells were plated in 96-well plates at $10^{3}$ cells/well and half of the media $(100 \mu \mathrm{L})$ were collected to measure the amount of glutamate at indicated time. Cells in the remaining half of the media $(100 \mu \mathrm{L})$ were used in MTT cell proliferation assays (Bottom). Media without cells were used in each experiment to compensate for the quality of measurements. At least three independent experiments were done and representative graphs are shown here. The graph in the middle is the same data as the top graph, but an expended scale. All human melanoma cells, especially C8161, displayed elevated levels of extracellular glutamate in comparison to the control, HEK. 


\section{C8161}
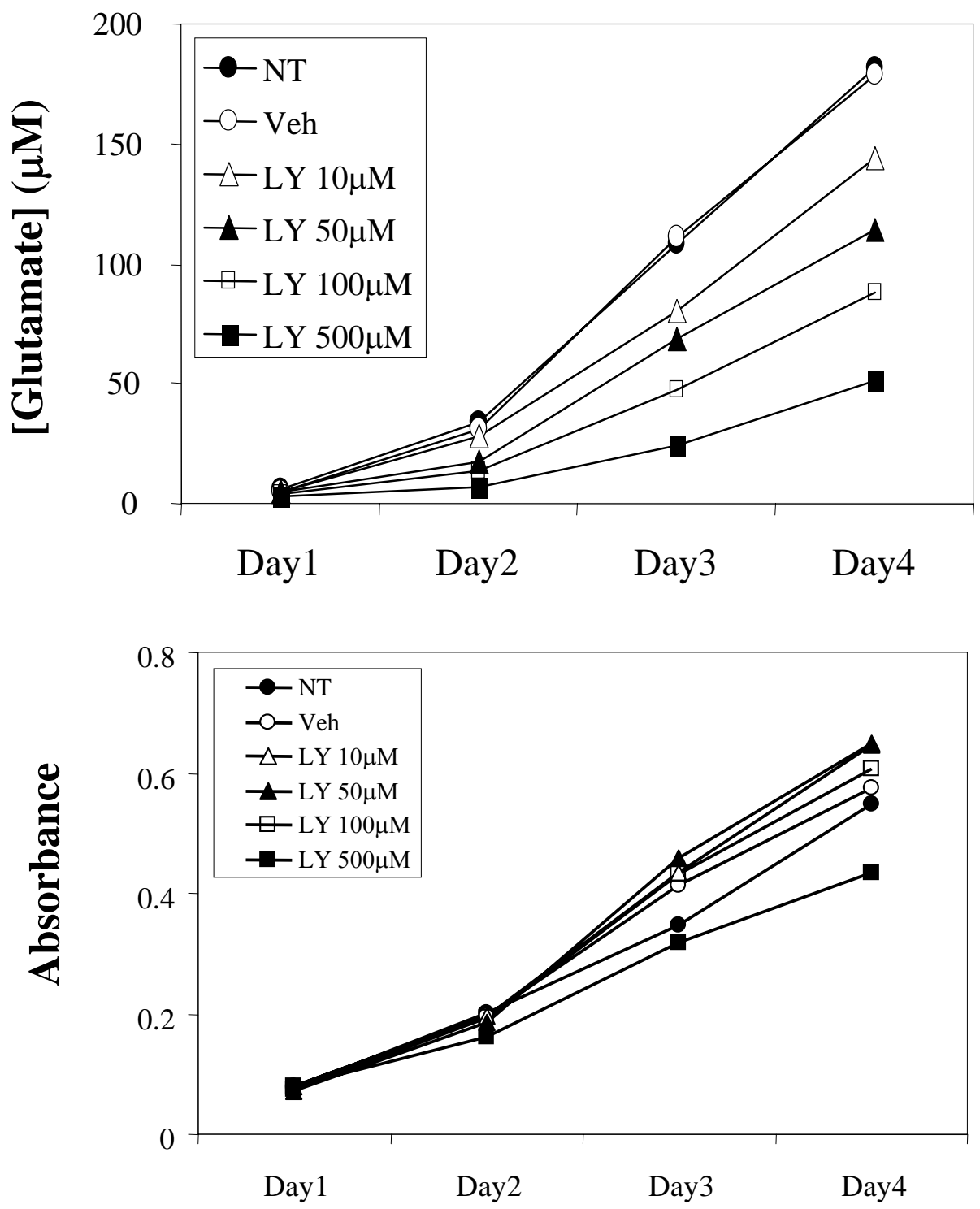

Figure 33

Glutamate Release Measurements of LY367385 treated C8161

The ability of the competitive GRM1 antagonists, LY367385, to inhibit the release of glutamate was examined in C8161 at different concentrations for up to four days (Top graph). Three independent experiments were done and representative graphs are shown here. A dose-dependent inhibition of release of extracellular glutamate was observed only in LY367385 treated C8161, while no inhibition was detected in vehicle treated cells. MTT cell proliferation assay is shown (Bottom graph). 


\section{HEK293A}
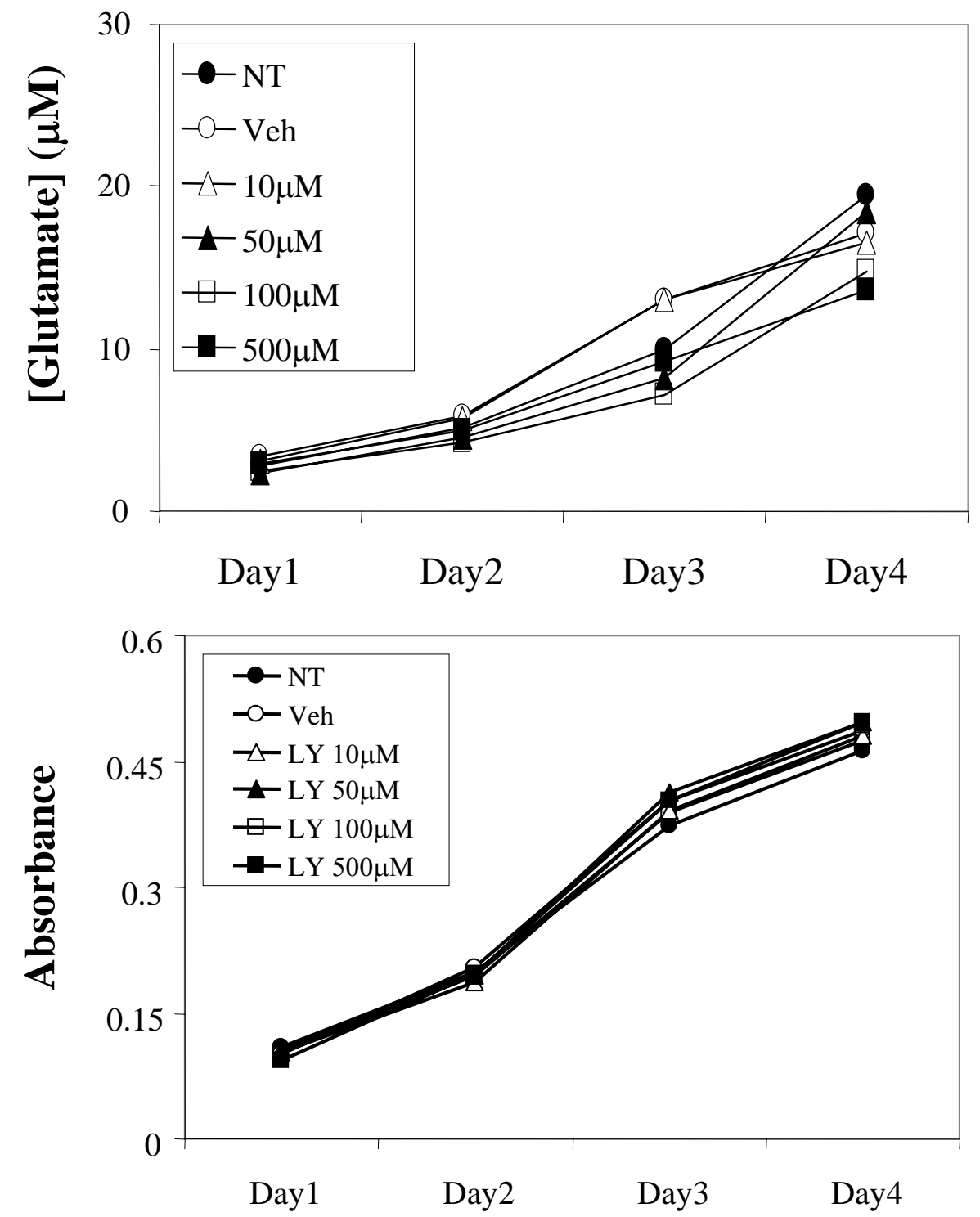

Figure 34

Glutamate Release Measurements of LY367385 treated HEK293A

The ability of the competitive GRM1 antagonists, LY367385, to inhibit the release of glutamate was examined in HEK293A at different concentrations for up to four days (Top graph). At least three independent experiments were done and representative graphs are shown here. There was little or no difference of the amount of extracellular glutamate in LY367385 treated HEK293A. MTT cell proliferation assay is shown (Bottom graph). 

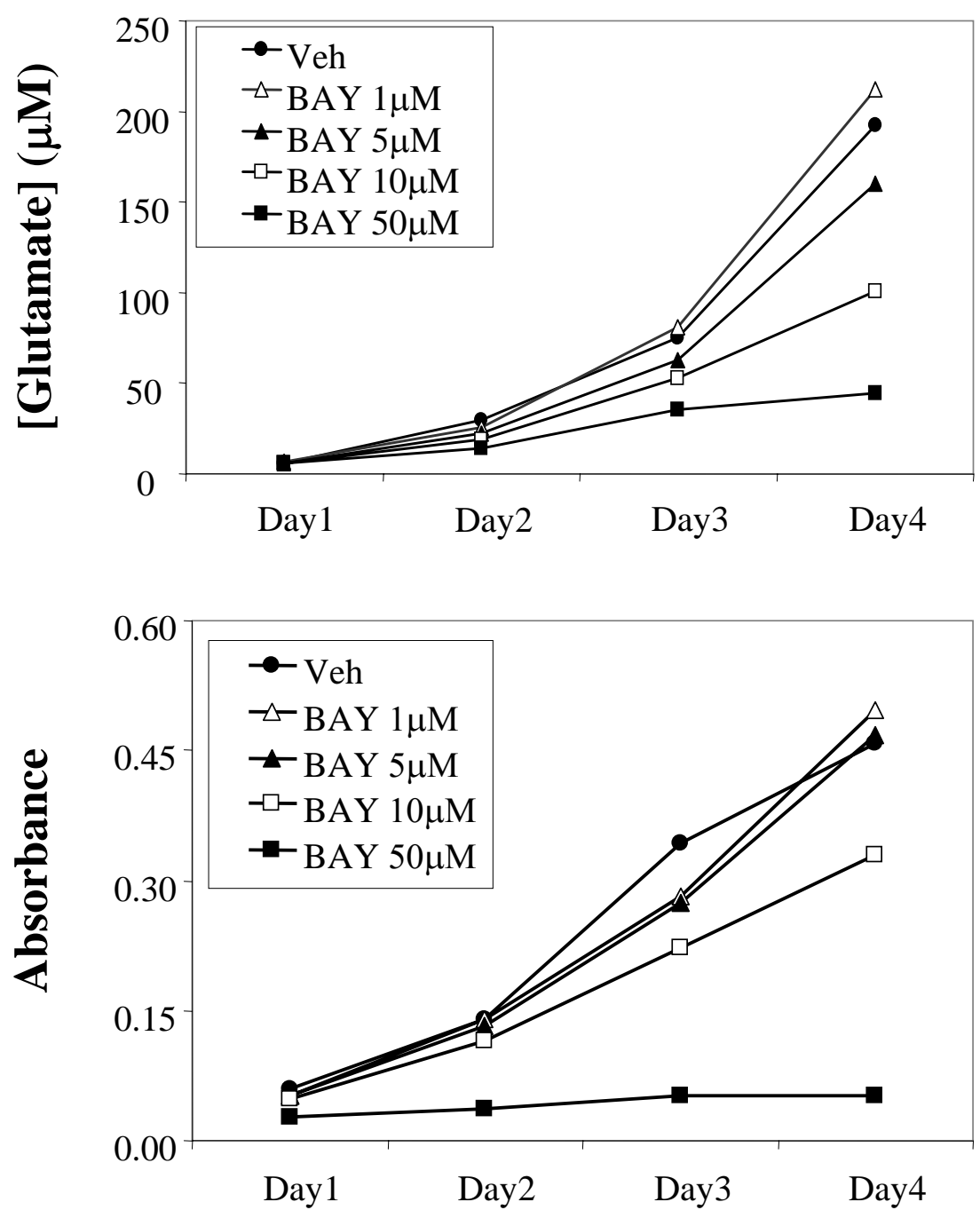

Figure 35

Glutamate Release Measurements of BAY 36-7620 treated C8161

The ability of the non-competitive GRM1 antagonists, BAY 36-7620, to inhibit the release of glutamate was examined in C8161 at different concentrations for up to four days (Top graph). The vehicle control was DMSO. At least three independent experiments were done and representative graphs are shown here. A dose-dependent inhibition of release of extracellular glutamate was observed only in BAY 36-7620 treated C8161, while no inhibition was detected in vehicle treated cells. MTT cell proliferation assay is shown (Bottom graph). 

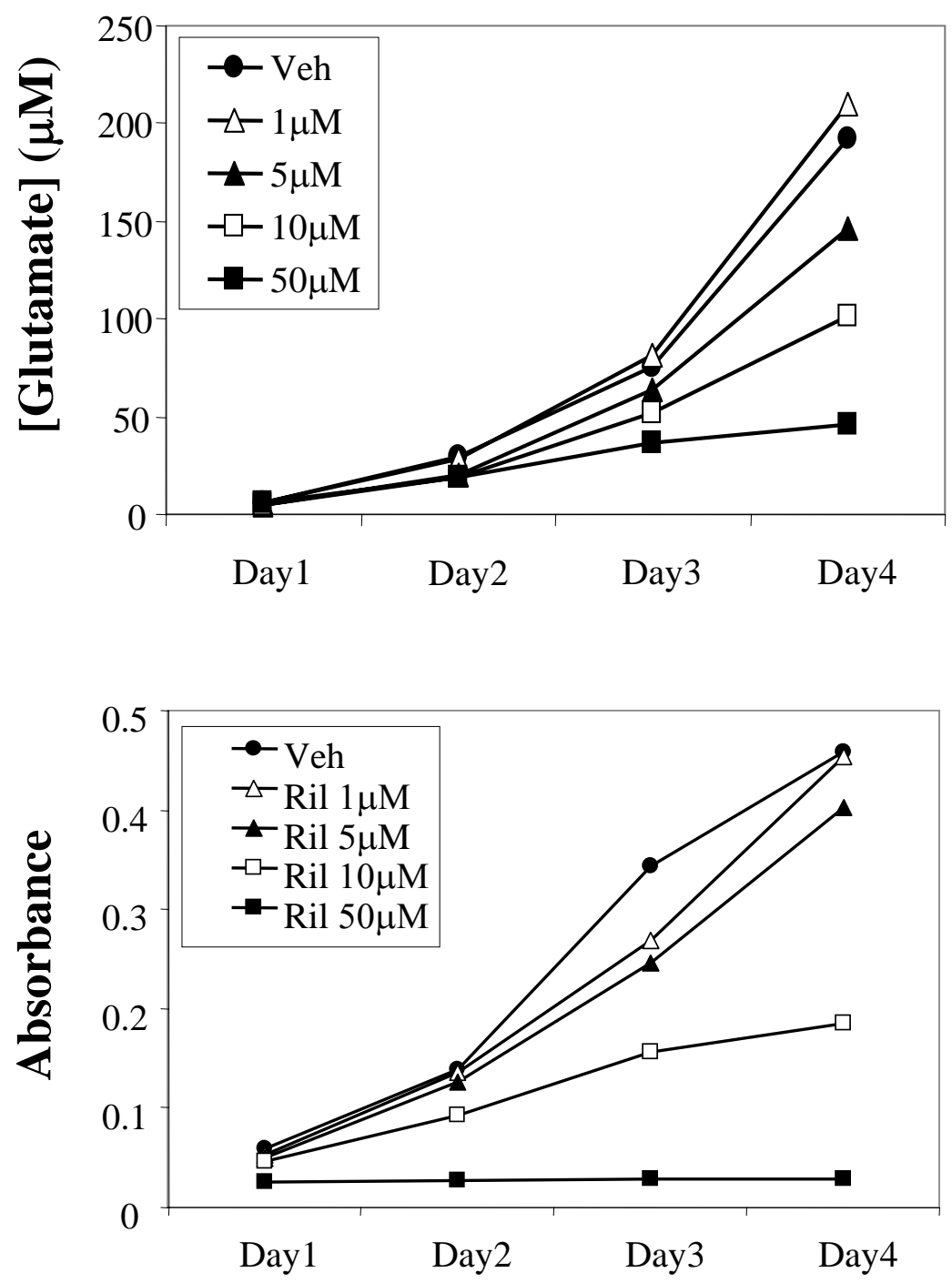

Figure 36

Glutamate Release Measurements of Riluzole treated C8161

The ability of the glutamate release inhibitor, Riluzole, to inhibit the release of glutamate was examined in C8161 at different concentrations for up to four days (Top graph). The vehicle control was DMSO. At least three independent experiments were done and representative graphs are shown here. A dose-dependent inhibition of release of extracellular glutamate was observed only in Riluzole treated C8161, while no inhibition was detected in vehicle treated cells. MTT cell proliferation assay is shown (Bottom graph). 


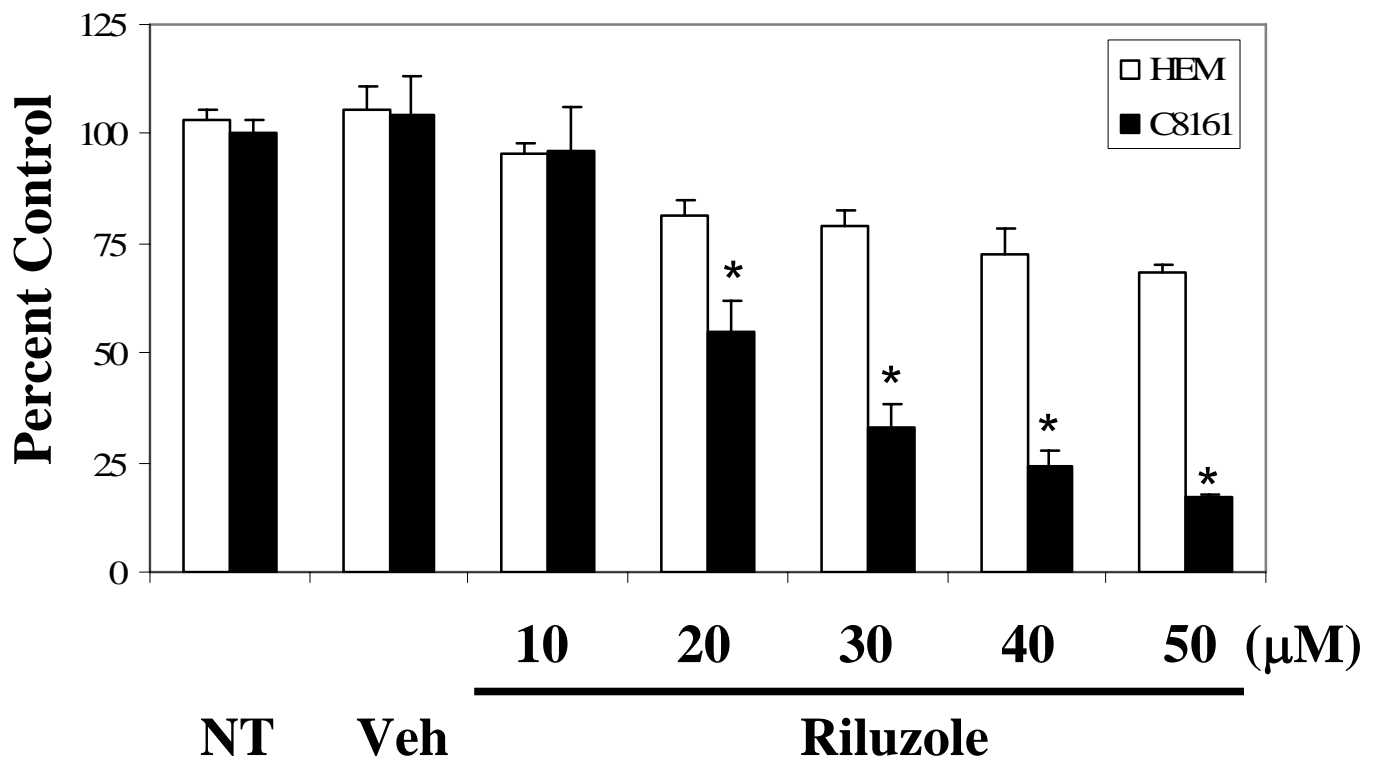

Figure 37

MTT Cell Proliferation Assay with the Glutamate Release Inhibitor, Riluzole

MTT cell proliferation assays were used to assess the biological consequences of C8161 (black bar) and HEM (white bar) treated with Riluzole. Cells were plated on 96-well plate at $10^{3}$ cells/well and treated with 10, 20, 30, 40 or $50 \mu \mathrm{M}$ Riluzole. Each point represents the average of quadruplicates. Minimum of three independent experiments were done and a representative graph is shown here. A dose-dependent suppression of C8161 cell growth by Riluzole was detected in comparison to no treatment (NT) and DMSO treated (Veh). Only day 4 measurements are shown here. HEM cells were less sensitive to Riluzole under similar conditions. Bar, SD; *, $\mathrm{P}<0.001$ compared to HEM (t-test). 


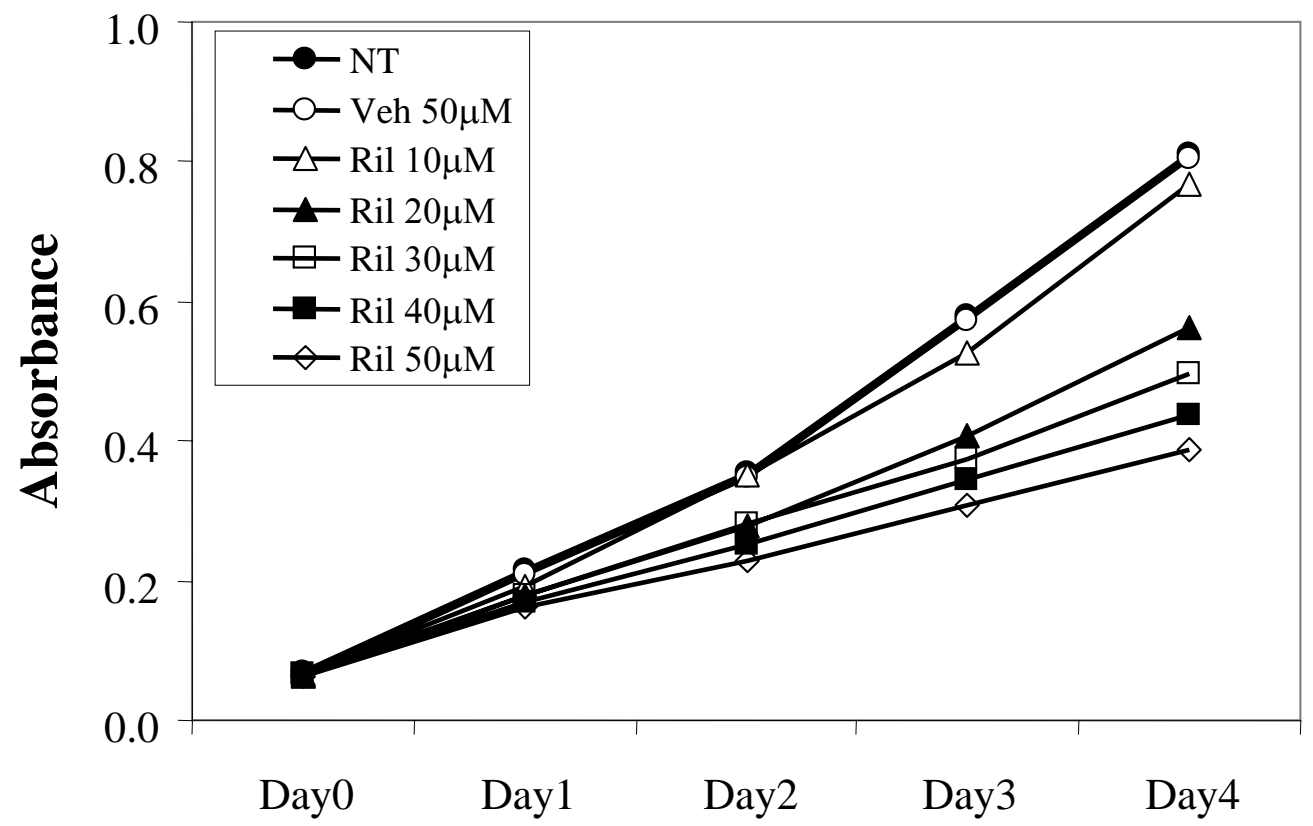

Figure 38

MTT Cell Proliferation Assay of Riluzole treated WM239A

Biological responses of human melanoma cells to Riluzole were measured on WM239A human melanoma cell line. Cells were treated with Riluzole at concentrations of 10, 20, 30, 40 and $50 \mu \mathrm{M}$ respectively. Each point represents the average of quadruplicates. Three independent experiments were done and a representative graph is shown here. No treatment (NT) and $50 \mu \mathrm{M}$ DMSO (Vehicle) were used as controls. Measurement of cell viability/growth was carried out by MTT assays for four days. Riluzole had growth inhibitory effect on WM239A cells in a dose-dependent manner. 


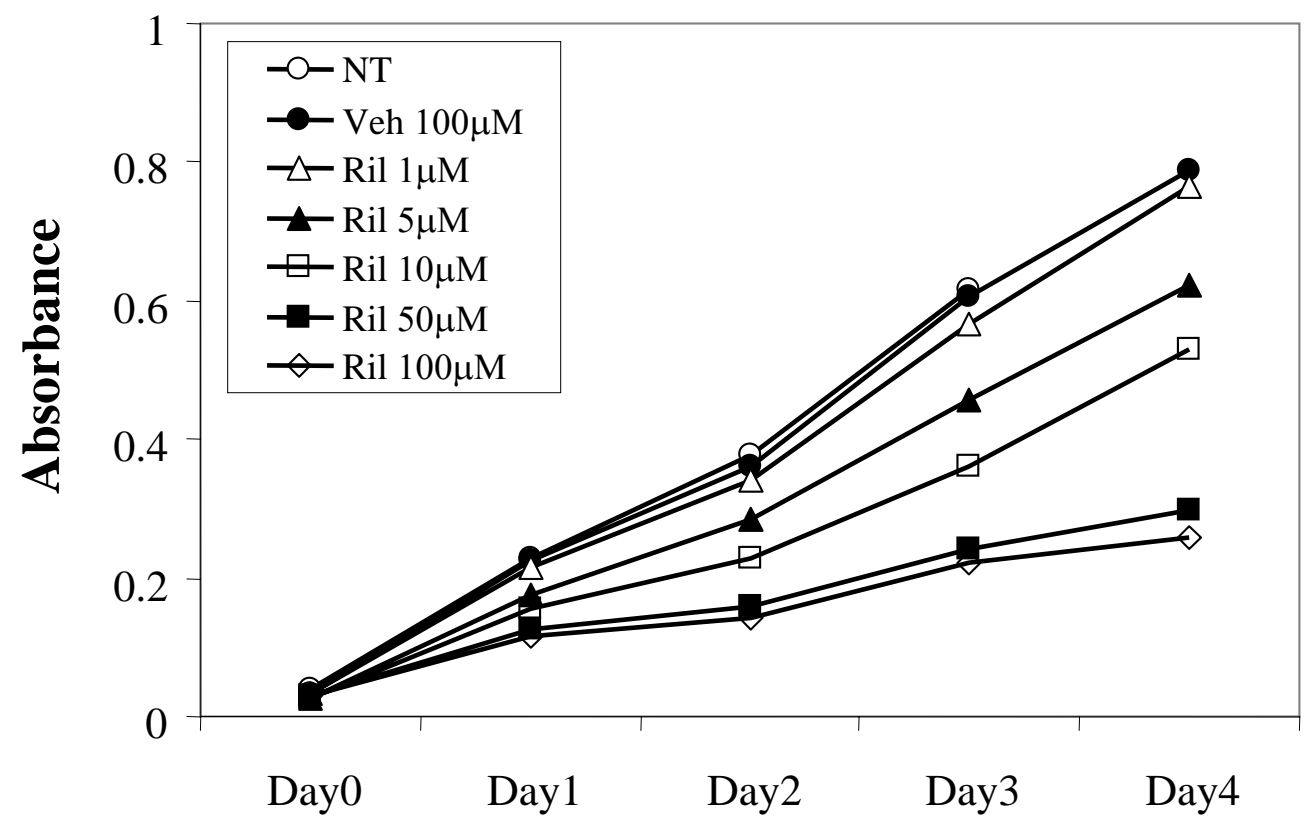

Figure 39

MTT Cell Proliferation Assay of Riluzole treated UACC903

Biological responses of human melanoma cells to Riluzole were measured on UACC903 human melanoma cell line. Cells were treated with Riluzole at concentrations of 1, 5, 10, 50 and $100 \mu \mathrm{M}$ respectively. Each point represents the average of quadruplicates. Three independent experiments were done and a representative graph is shown here. No treatment (NT) and $100 \mu \mathrm{M}$ DMSO (Vehicle) were used as controls. Measurement of cell viability/growth was carried out by MTT assays for four days. Riluzole had growth inhibitory effect on UACC903 cells in a dose-dependent manner. 
24 Hours

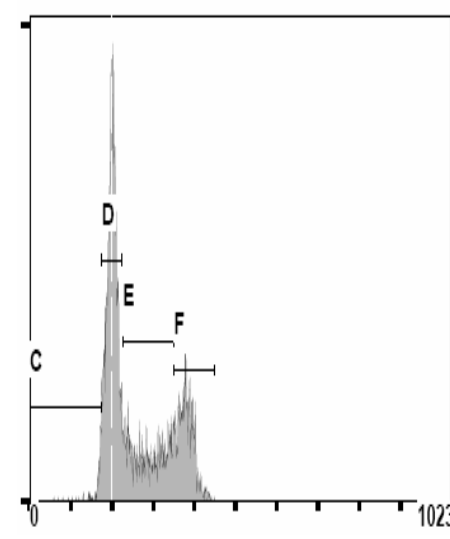

No Treatments

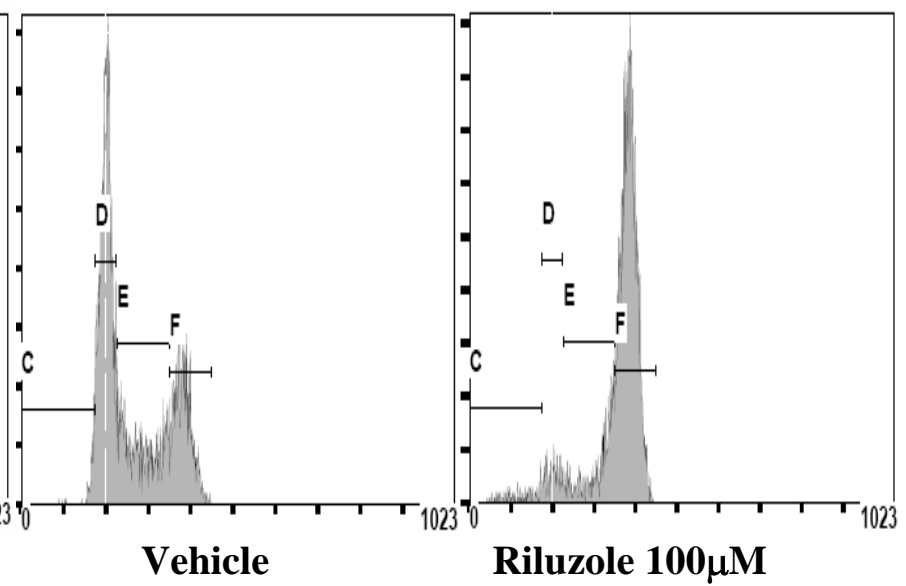

48 Hours

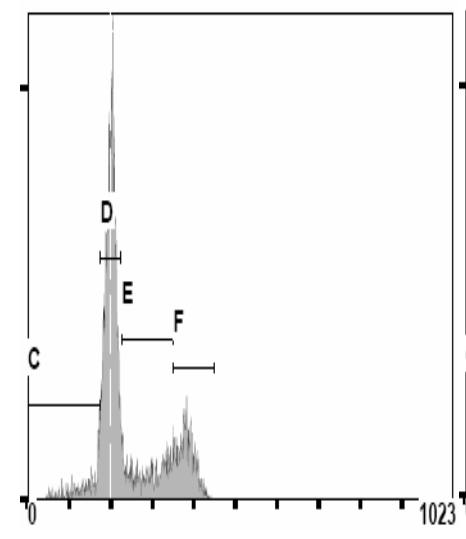

No Treatments

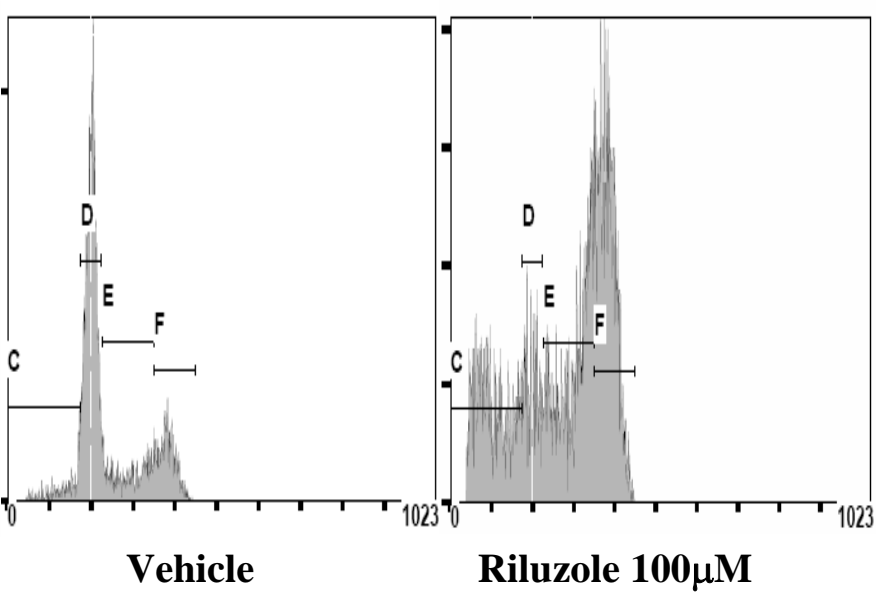

Figure 40

Cell Cycle Analysis of Riluzole treated C8161

Cell cycle analysis were performed with C8161 cells treated with Riluzole at 24 (top) and 48 (bottom) hours. At 24 hours post-treatment, cells were accumulated in the G2/M phase of the cell cycle. At 48 hours post-treatment, a three-fold increase in the sub-G1 phase of the cell cycle was detected. DMSO treatment (Vehicle) had little or no effects. Each phase of cell cycle was indicated as C (sub-G1), D (G1), E (S) and F (G2/M) and the percentage of cells in each phase was given in Table 4 (24 hours) and Table 5 (48 hours). 


\section{WM239A}

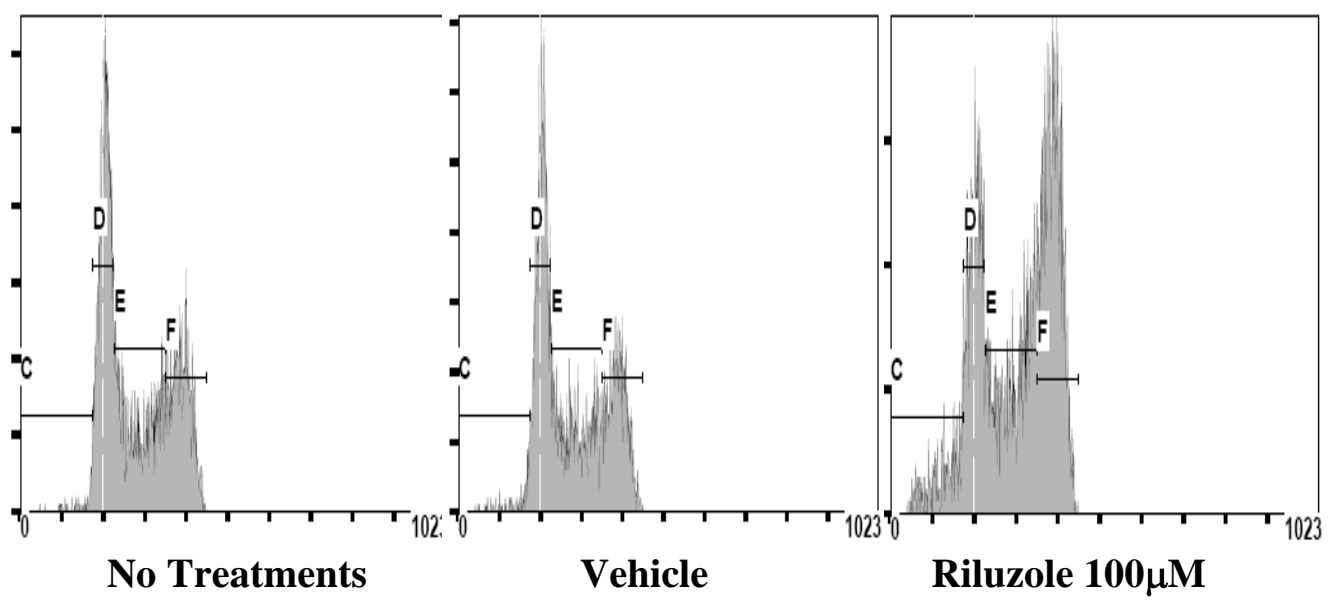

Figure 41

Cell Cycle Analysis of Riluzole treated WM239A

Cell cycle analysis were performed with WM239A cells treated with Riluzole at 24 hours. At 24 hours post-treatment, cells were accumulated in the sub-G1 and G2/M phases of the cell cycle. DMSO treatment (Vehicle) had little or no effects. Each phase of cell cycle was indicated as C (sub-G1), D (G1), E (S) and F (G2/M) and the percentage of cells in each phase was given in Table 7. 
24 Hours

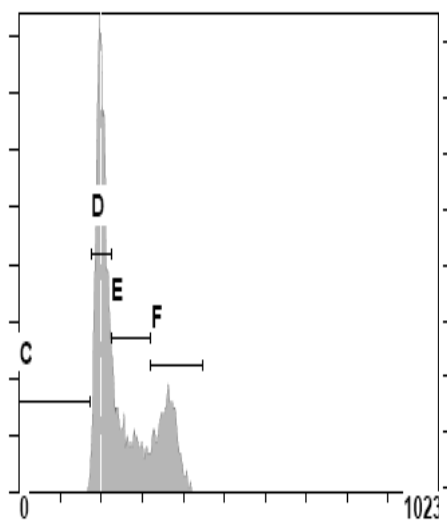

No Treatments

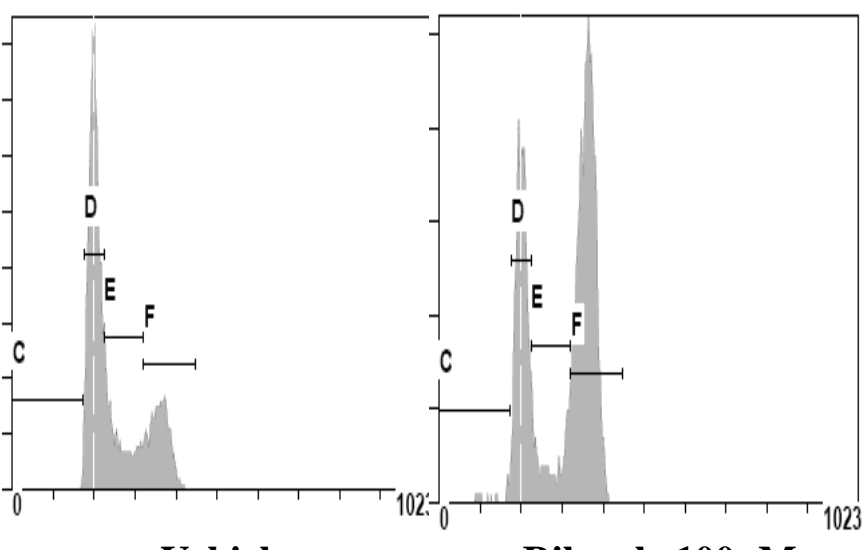

Vehicle

Riluzole $100 \mu \mathrm{M}$

48 Hours

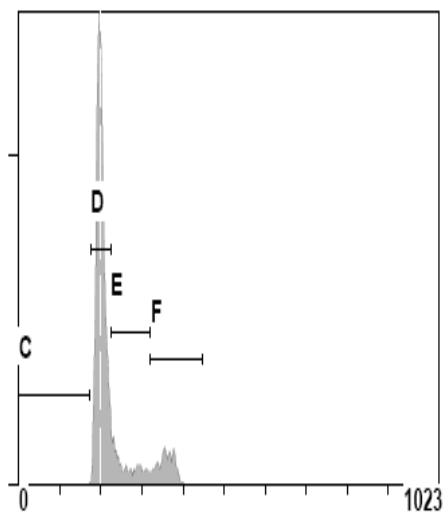

No Treatments

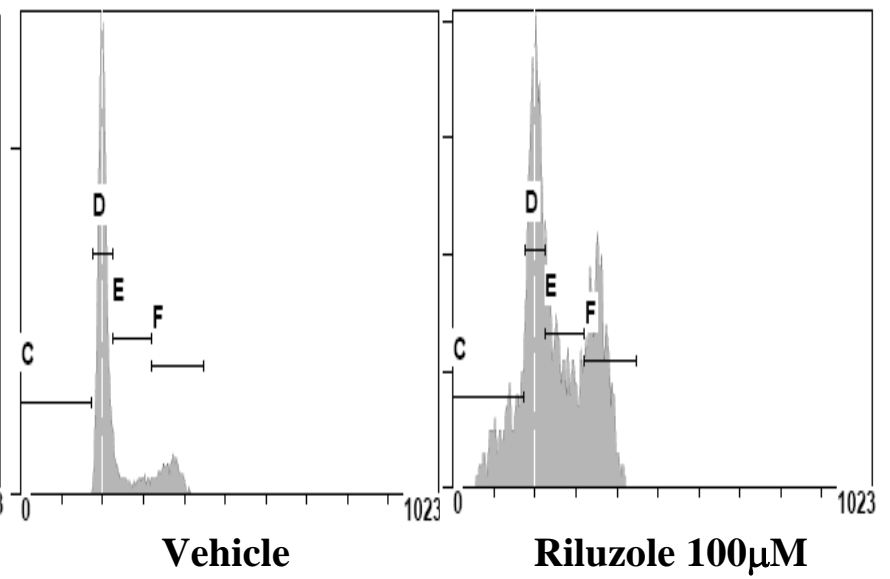

Figure 42

Cell Cycle Analysis of Riluzole treated UACC903

Cell cycle analysis were performed with UACC903 cells treated with Riluzole at 24 (top) and 48 (bottom) hours. At 24 and 48 hours post-treatment, cells were accumulated in the sub-G1 and G2/M phases of the cell cycle. DMSO treatment (Vehicle) had little or no effects. Each phase of cell cycle was indicated as $\mathrm{C}$ (subG1), D (G1), E (S) and F (G2/M) and the percentage of cells in each phase was given in Table 10 (24 hours) and Table 11 (48 hours). 


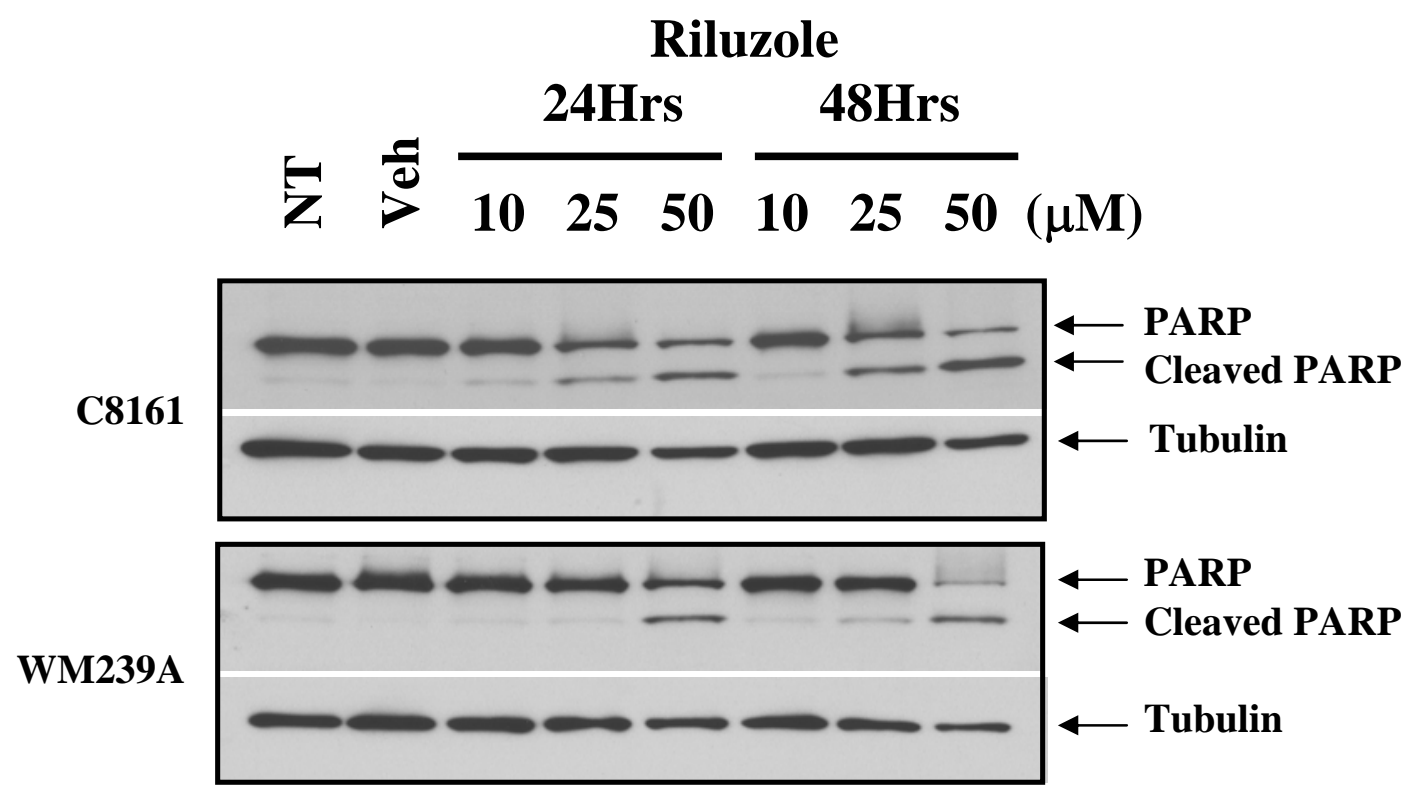

Figure 43

Induction of Apoptosis by Riluzole Treatments

To investigate the apoptotic response of C8161 and WM239A human melanoma cells to Riluzole, cells were plated and treated with 10,25 or $50 \mu \mathrm{M}$ Riluzole for 24 or 48 hours. Protein lysates were extracted for western immunoblots. Three independent experiments were done and representative blots are shown here. After 24 and 48 hours of Riluzole treatments, apoptosis was induced in C8161 cells as evident by PARP cleavage compared to no treatment (NT) or DMSO treated (Veh). The same blot was probed with tubulin to show equal loading. 


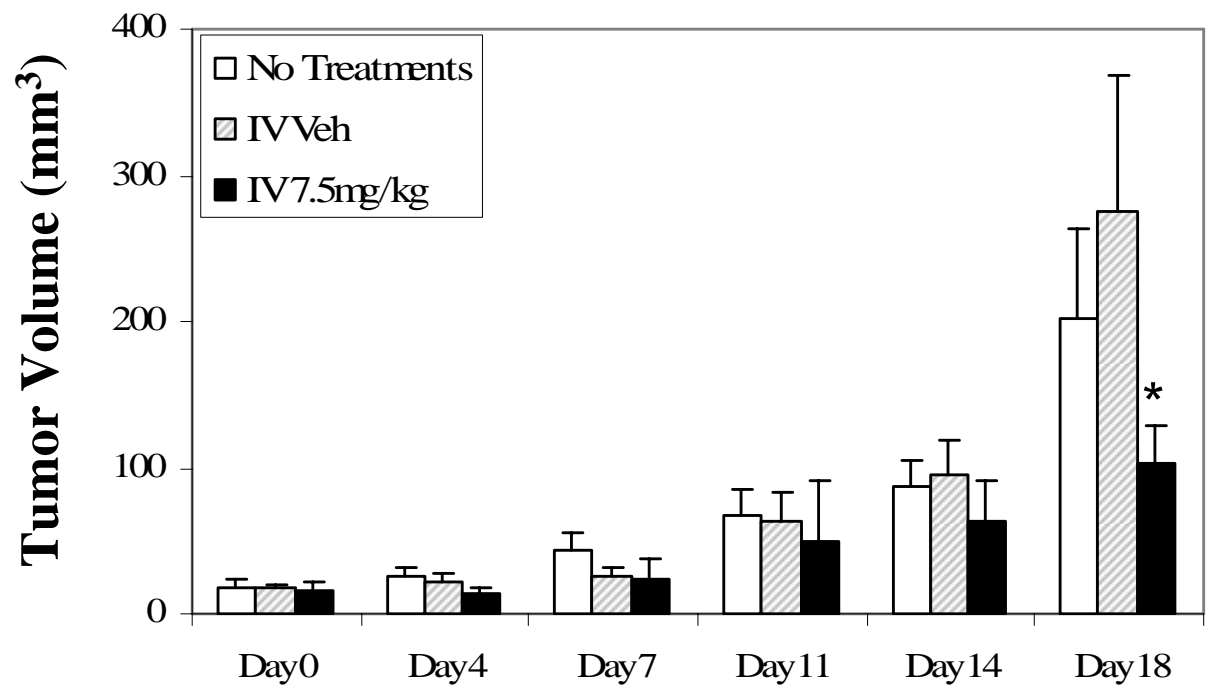

Figure 44

C8161 Xenograft Model for Riluzole IV Treatment

Therapeutic potential of Riluzole was investigated by C8161 xenograft model. C8161 human melanoma cells were inoculated subcutaneously in 6-week old nude mice at $10^{6}$ cells/site. When the tumor volumes had reached $6 \mathrm{~mm}^{3}$, mice were treated with 7.5 $\mathrm{mg} / \mathrm{kg}$ Riluzole daily by IV. Tumor sizes were measured twice weekly with a vernier caliper. Treatments were terminated after 18 days. Whether administered by IV or oral gavage, a reduction in the tumor volumes was detected in Riluzole treated mice in comparison to untreated (NT) or DMSO treated (Veh) controls. Bar, SD; *, P<0.01 compared to NT and Veh (t-test). 


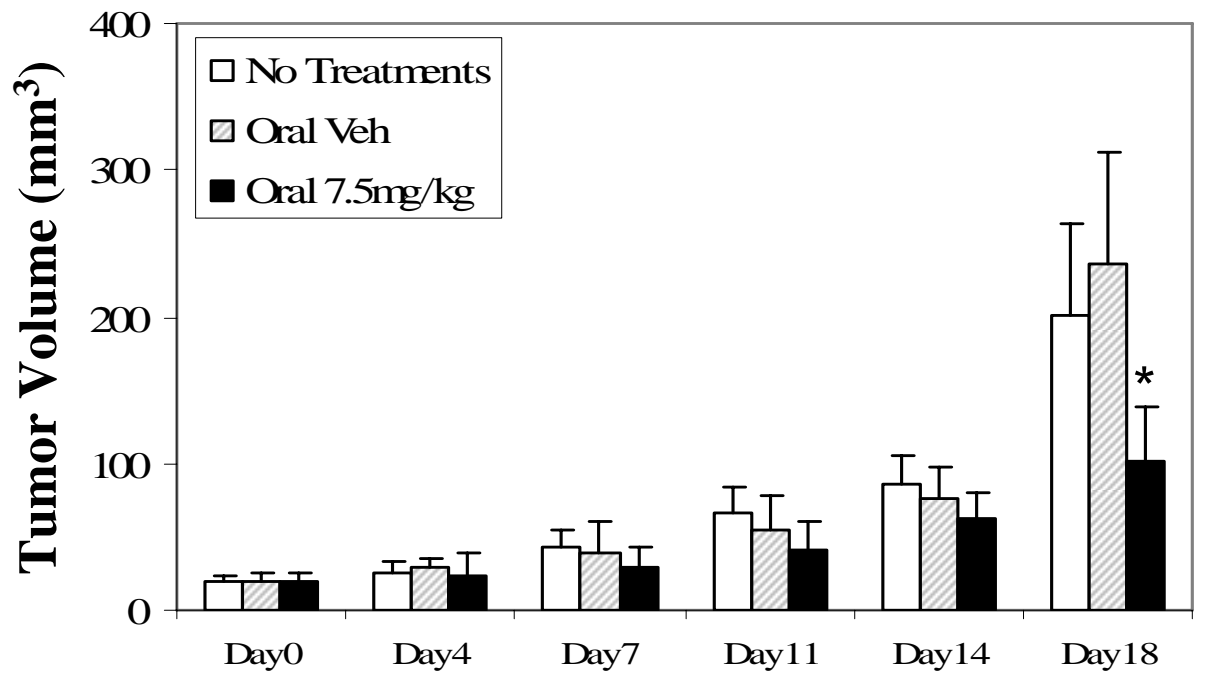

Figure 45

C8161 Xenograft Model for Riluzole Oral Treatment

Therapeutic potential of Riluzole was investigated by C8161 xenograft model. C8161 human melanoma cells were inoculated subcutaneously in 6-week old nude mice at $10^{6}$ cells/site. When the tumor volumes had reached $6 \mathrm{~mm}^{3}$, mice were treated with 7.5 $\mathrm{mg} / \mathrm{kg}$ Riluzole daily by oral gavage. Tumor sizes were measured twice weekly with a vernier caliper. Treatments were terminated after 18 days. Whether administered by IV or oral gavage, a reduction in the tumor volumes was detected in Riluzole treated mice in comparison to untreated (NT) or DMSO treated (Veh) controls. Bar, SD; *, $\mathrm{P}<0.01$ compared to NT and Veh (t-test). 

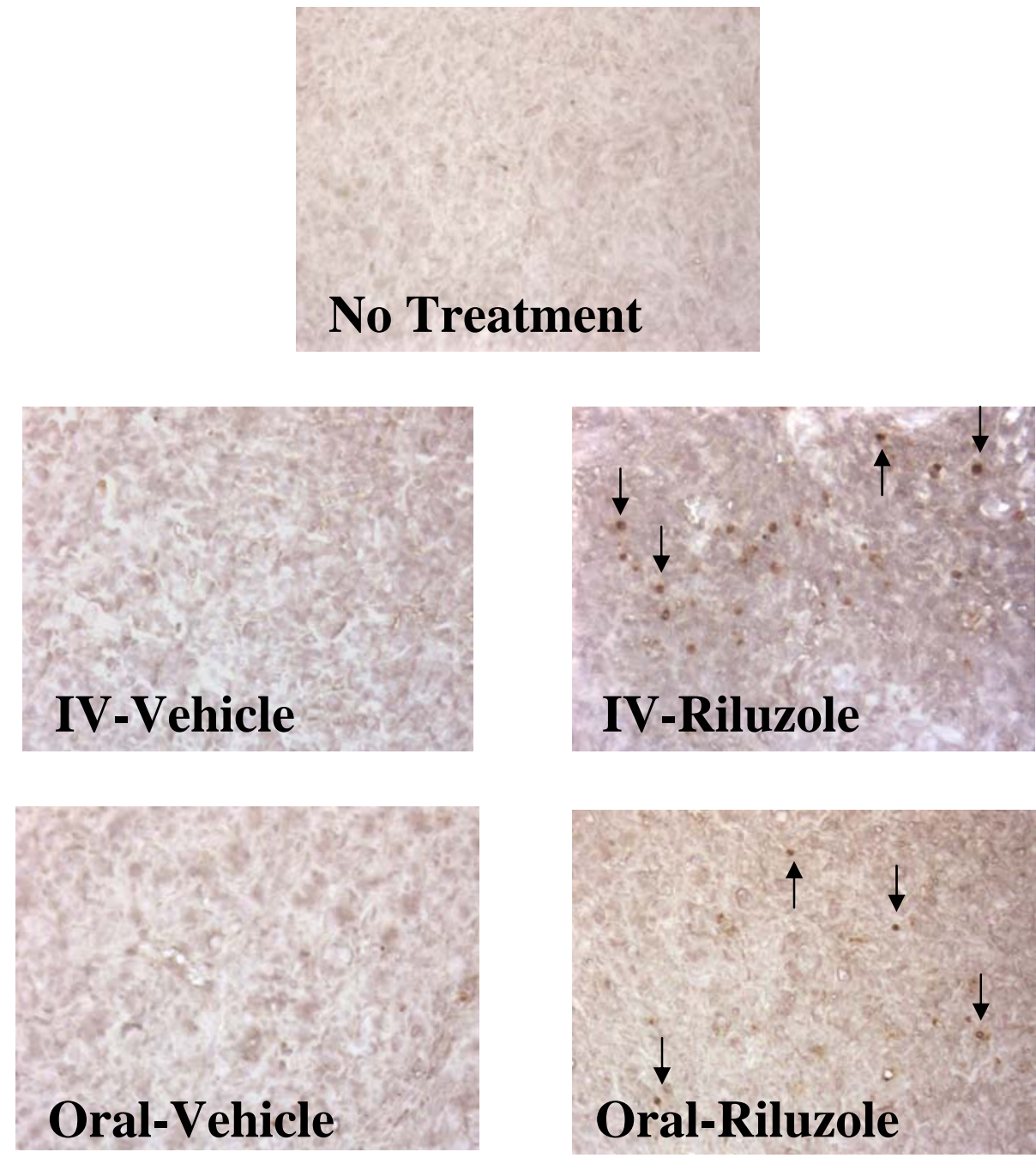

Figure 46

Immunohistochemistry: C8161 Xenograft Model for Riluzole Treatment

To investigate whether Riluzole induce apoptosis in C8161 xenograft model, tumor was excised and fixed for immunohistochemistry. ApopTag Plus Peroxidase In Situ apoptosis detection kit was used to detect apoptotic cells. Compared to no treatment or respective vehicles, Riluzole treated tissues had more positively stained apoptotic cells. 


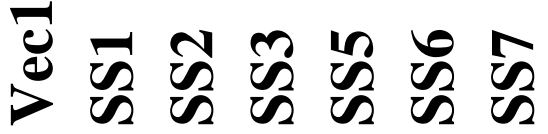

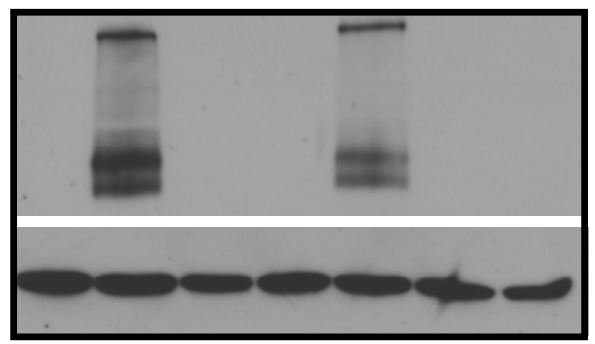

Grm1

Tubulin

Figure 47

UACC930-Grm1 Stable Clones: Western

UACC930 is a human melanoma cell line with a truncation mutation in GRM1 coding region. In order to investigate whether GRM1 could be an advantage to cell growth of UACC930, stable clones were established using mouse Grm1 cDNA under the control of CMV promoter (SS) and vector control (Vec). A representative western immunoblot showed two clones (SS1 and SS5) express Grm1. Tubulin was used as a loading control. 

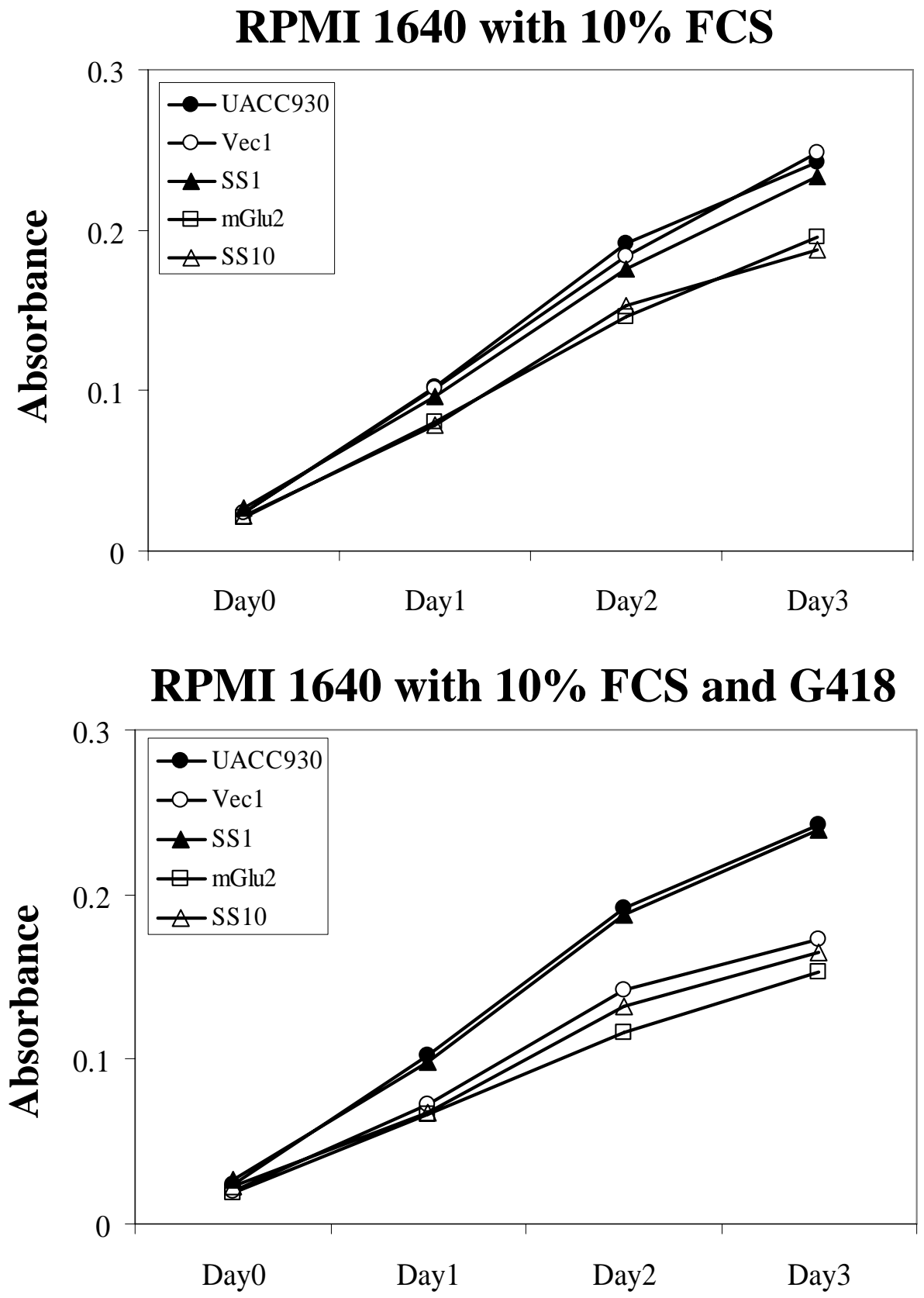

Figure 48

UACC930-Grm1 Stable Clones: MTT Cell Proliferation Assay

MTT assay was performed on UACC930 cells transfected with Grm1 cDNA (SS or mGlu) or vector control (Vec) without G418 (Top) or with G418 (Bottom). Each point represents the average of quadruplicates. Cells transfected with Grm1 show no advantage for cell growth when compared to the parent control. However, when compared to vector control, slight growth advantage is demonstrated. 


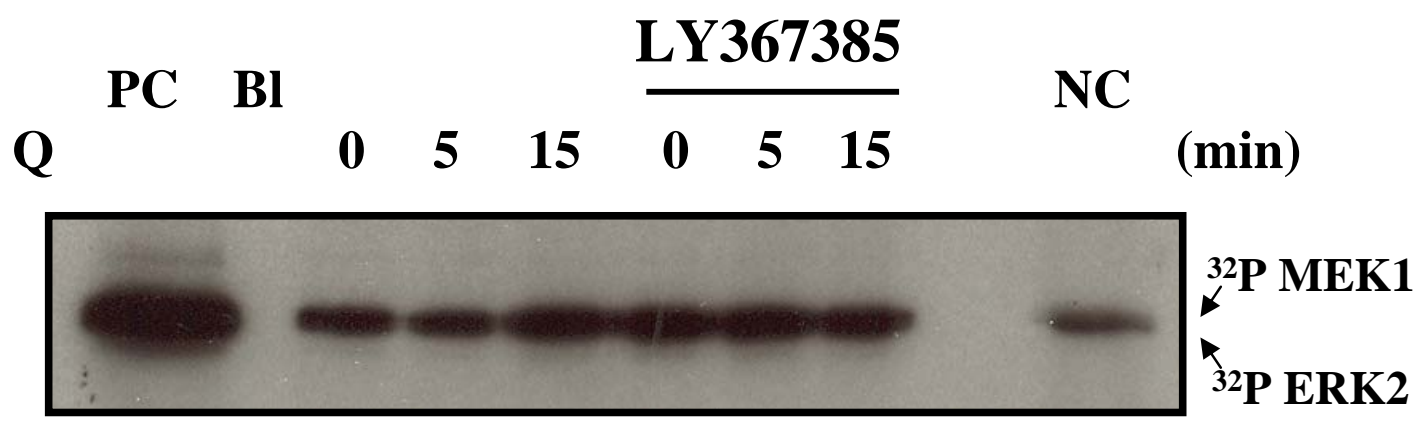

Figure 49

BRAF Kinase Assay-32 $\mathrm{P}$

In order to investigate the involvement of BRAF in Grm1 signaling pathway, BRAF kinase assay was done using Nu2 (TG3 mouse melanoma cell line). Cells were treated with L-quisqualate (Q) for 5 or 15 minutes or pretreated with LY367385 for 30min prior to Q induction. Positive control (PC) contains activated BRAF from the Upstate kit and negative control (NC) contains buffers. ${ }^{32} \mathrm{P}-\gamma$-ATP was used for radioactivity. The gel was exposed to film overnight. No significant changes were detected. 


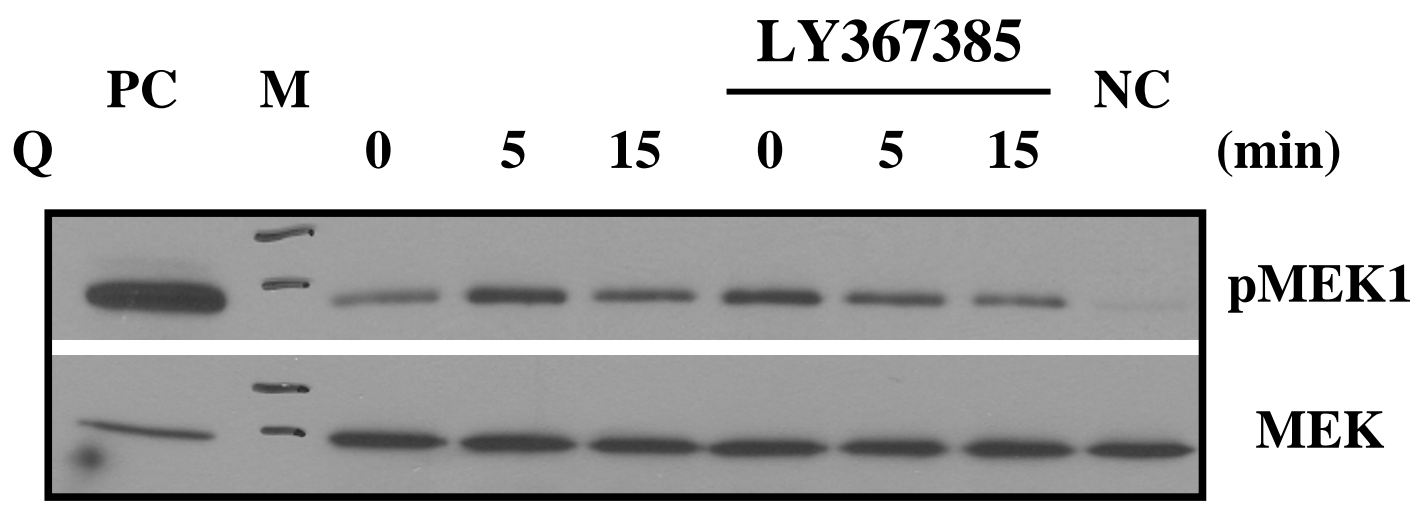

Figure 50

BRAF Kinase Assay-Western Blot

In order to investigate the involvement of BRAF in Grm1 signaling pathway, BRAF kinase assay was done using Nu2 (TG3 mouse melanoma cell line). Cells were treated with L-quisqualate (Q) for 5 or 15 minutes or pretreated with LY367385 for 30min prior to Q induction. Positive control (PC) contains activated BRAF from the Upstate kit and negative control (NC) contains buffers. Instead of radioactivity, western was performed and probed with phospho-MEK1. The membrane was stripped and reprobed with MEK for normalization. Slight increase in MEK1 phosphorylation is shown after 5 minutes of $\mathrm{Q}$ induction. 


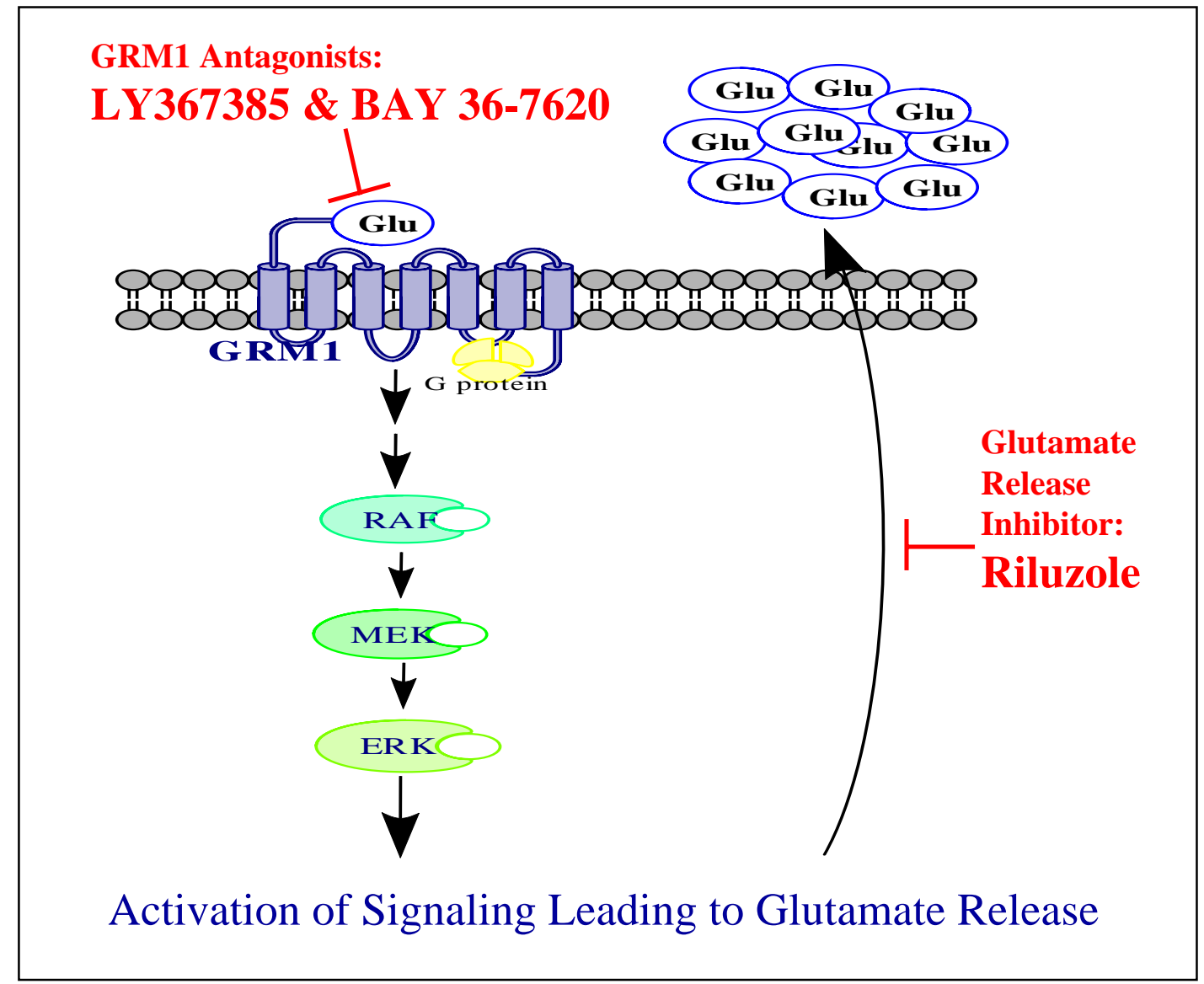

Figure 51

Proposed GRM1 Signaling in Human Melanoma

Upon the stimulation of GRM1 by its ligand, glutamate, GRM1 activates the downstream signaling pathway leading to ERK phosphorylation. Phosphorylated ERK will lead to the melanoma cell proliferation. In addition, the signaling cascade activated by GRM1 stimulation leads to release of glutamate, which in turn, will further stimulate GRM1. Suppressing GRM1 signaling by GRM1 specific antagonists prevent this signaling cascade and Riluzole, a glutamate release inhibitor, suppresses the release of glutamate and prevents the further stimulation of GRM1. 


\title{
CURRICULUM VITA
}

\section{JIN NAMKOONG}

\author{
Education \\ 2000-2007 \\ Rutgers University-UMDNJ Joint Program in Molecular Bioscience \\ Ph.D. in Microbiology and Molecular Genetics \\ 2006 \\ 2001-2005 \\ The NCI sponsored Tumor Microenvironment Training Program \\ 2000-2001 \\ Teaching Assistantship, Department of Chemical Biology \\ Ernest Mario School of Pharmacy, Rutgers University \\ Graduate Fellow, Rutgers University-UMDNJ Joint Program in \\ Molecular Bioscience \\ 1995-1999 Cook College, Rutgers University, New Brunswick, NJ \\ Bachelor of Science in Biochemistry
}

\section{Publications}

Namkoong, J., Shin, S. S., Lee, H., Marin, Y. E., Goydos, J., and Chen, S., 2007. Metabotropic Glutamate Receptor 1 and Glutamate Signaling in Human Melanoma. Cancer Research. 67, 2298-2305.

Namkoong, J., Martino, J. J., and Chen, S. 2006. From Existing Therapies to Novel Targets: a Current View on Melanoma. Frontiers in BioScience. 11, 2081-2092.

Marin, Y. E., Namkoong, J., Cohen-Solal, K., Shin, S. S., Martino, J. J., Oka, M., and Chen, S. 2006. Stimulation of Metabotropic Glutamate Receptor 1 in Melanoma Cells activates ERK1/2 via PKCE. Cellular Signaling. 18, 1279-1286.

Marin, Y. E., Namkoong, J., Shin, S. S., Raines, J., Degenhardt, K., White, E., and Chen, S. 2005. Grm5 Expression is not required for the Oncogenic Role of Grm1 in Melanocytes. Neuropharmacology. 49 Suppl, 70-79.

Sood, R., Makalowska, I., Galdzicki, M., Hu, P., Eddings, E., Robbins, C. M., Moses, T., Namkoong, J., Chen, S. and Trent, J. M. 2003. Cloning and characterization of a novel gene, SHPRH, encoding a conserved putative protein with SNF2/helicase and PHD-finger domains from the 6q24 region. Genomics. 82, 153-161.

Pollock, P. M., Cohen-Solal, K., Sood, R., Namkoong, J., Martino, J. J., Koganti, A., Zhu, H., Robbins, C., Makalowska, I., Shin, S. S., Marin, Y., Roberts, K. G., Yudt, L. M., Chen, A., Cheng, J., Incao, A., Pinkett, H. W., Graham, C. L., Dunn, K., Crespo-Carbone, S. M., Mackason, K. R., Ryan, K. B., Sinsimer, D., Goydos, J., Reuhl, K. R., Eckhaus, M., Meltzer, P. S., Pavan, W. J., Trent, J. M. and Chen, S. 2003. Melanoma mouse model implicates metabotropic glutamate signaling in melanocytic neoplasia. Nature Genetics. 34, 108-112. 
Cohen-Solal, K. A., Crespo-Carbone, S. M., Namkoong, J., Mackason, K. R., Roberts, K. G., Reuhl, K. R. and Chen, S. 2002. Progressive appearance of pigmentation in amelanotic melanoma lesions. Pigment Cell Research 15, 282-289. 